\title{
MEASURES ON CANTOR SETS: THE GOOD, THE UGLY, THE BAD
}

\author{
SERGEY BEZUGLYI AND DAVID HANDELMAN
}

\begin{abstract}
We translate Akin's notion of good (and related concepts) from measures on Cantor sets to traces on dimension groups, and particularly for invariant measures of minimal homeomorphisms (and their corresponding simple dimension groups). This yields characterizations and examples, which translate back to the original context. Good traces on a simple dimension group are characterized by their kernel having dense image in their annihilating set of affine functions on the trace space; this makes it possible to construct many examples with seemingly paradoxical properties.

In order to study the related property of refinability, we consider goodness for sets of measures (traces on dimension groups), and obtain partial characterizations in terms of (special) convex subsets of Choquet simplices.

These notions are also very closely related to unperforation of quotients of dimension groups by convex subgroups (that are not order ideals), and we give partial characterizations. Numerous examples illustrate the results.
\end{abstract}

\section{INTRODUCTION}

Let $X$ be a totally disconnected (that is, zero-dimensional) separable compact set with no isolated points; we refer to it as a Cantor set. Let $\mu$ be a probability measure on $X$. Motivated by the problem of homeomorphism of measures, Akin initiated a systematic study of Borel probability measures on a Cantor set [Aki99, Aki05. He showed that the clopen value set $S(\mu)$ (the set of values of $\mu$ on clopen sets) plays a significant role in classification of measures up to a homeomorphism. It is a countable dense subset of the unit interval, and this set provides an invariant for topologically equivalent measures, although not complete. However, for the class of good measures, $S(\mu)$ is a complete invariant. By definition, a (full nonatomic) probability measure $\mu$ is good if whenever $U, V$ are clopen sets with $\mu(U)<\mu(V)$, there exists a clopen subset $W$ of $V$ such that $\mu(W)=\mu(U)$. It turns out that such measures are exactly the invariant measures which can arise in uniquely ergodic minimal homeomorphisms of Cantor sets [Aki05, GW95.

A series of papers in the last decade, by Akin, Austin, Dougherty, Mauldin, Yingst (ADMY08, Aus07, DMY07, Yin08), have focused on various properties

Received by the editors April 5, 2012 and, in revised form, September 16, 2012.

2010 Mathematics Subject Classification. Primary 19K14, 37B99, 46A55; Secondary 28C10, 14P10, 52A20, 06F20.

The second author was supported by an NSERC discovery grant.

The title of this paper is the correct translation of the title of the Italian spaghetti western ( $\mathrm{Il}$ buono, il brutto, il cattivo, D: Sergio Leone), which was changed for U.S. audiences. The English language title, The good, the bad, and the ugly, is clichéd now-over 175 articles in engineering and mathematics use it in their title-but we could not find any titles using the original order. 
of Bernoulli and related measures. In particular, the notion of (weak) refinability (weaker than good) was formulated and studied. More recently, BK11, Kar12. have exhaustively studied invariant finite and infinite measures of aperiodic substitution dynamical systems.

Akin Aki05] introduced the notion of a good measure, yielding criteria for two measures on $X$ to be homeomorphic (in the sense that there is a self-homeomorphism of $X$ sending one measure to the other). Specifically, if $\mu$ and $\mu^{\prime}$ are good probability measures, then they are homeomorphic if and only if their sets of values on clopen sets are equal. Glasner and Weiss GW95. showed that if there is a minimal homeomorphism $T$, and $A$ and $B$ are clopen sets such that $\mu(A)<\mu(B)$ for all invariant measures, then there exists an element, $\gamma$, of the topological full group of $(X, T)$ such that $\gamma(A) \subset B$. A particular consequence is that if $T$ is uniquely ergodic, then the measure is good.

Akin posed the question: if $\mu$ is a measure such that the group of homeomorphisms of $X$ that preserve $\mu$ acts minimally, then is $\mu$ good? If this were true, then all invariant measures of minimal homeomorphisms would be good. Early in this paper, we show that extremal (also known as ergodic or pure) invariant measures of a minimal homeomorphism need not be good, and are rarely so. For example, we can arrange examples wherein the extremal measures are not good, or the set of good pure measures and the set of not good pure measures are simultaneously dense in the set of pure (that is, ergodic) measures. We also introduce ugly and bad measures (formulated in terms of traces on dimension groups).

Our approach is to translate questions from the dynamical context, selfhomeomorphisms of Cantor sets, to the associated ordered abelian groups. When the action is minimal, the translation is complete; that is, for the problems associated with good measures, we can go back and forth between dynamical systems and simple dimension groups. In the nonminimal case, the situation is more complicated (this is discussed in the beginning of Section 1).

The language of dimension groups permits us to characterize good measures among the measures (now called traces) in terms of the ordered group and Choquet space structures, and this makes construction of examples relatively easy. For example, if $G$ is a simple dimension group with order unit $u$ and $\tau$ is a trace, then the trace $\tau$ is good (that is, when we translate back to invariant measures on a Cantor set with a minimal homeomorphism, the corresponding invariant measure is good) if and only if the image of $\operatorname{ker} \tau$ (a subgroup of $G$ ) has dense range in its vector space of affine functions that vanish at $\tau$.

Based on work of [GPS95] and before that of Vershik Ver81, we can go back and forth between invariant measures of minimal homeomorphisms of Cantor sets and traces of simple dimension groups. In fact, this translation can be extended to some nonminimal homeomorphism, via either Vershik's adic map, or via pre-ordered $K$ theory of crossed product $C^{*}$-algebras. In the former case, Medynets Med06 has shown that any aperiodic homeomorphism admits a representation as a Vershik map on (generally nonsimple) Bratteli diagrams. Dimension groups and Choquet theory have a large repertoire of results and examples, and it seems convenient to translate the original problems of measures to this context.

When $(X, T)$ is a Cantor set with a self-homeomorphism, we can form the usual $H^{1}(X, T)$ (Čech cohomology), which can be identified with $\mathrm{K}_{0}\left(C(X, \mathbf{C}) \times_{T} \mathbf{Z}\right)$, the pre-ordered Grothendieck group of the $C^{*}$-algebra crossed product. This can also 
be identified ([BH96], [Poo89], GPS95], Put89]) with the quotient group $G \equiv$ $G(X, T)=C(X, \mathbf{Z}) /(\mathrm{I}-T) C(X, \mathbf{Z})$ (the kernel representing the coboundaries) with the quotient pre-ordering (that is, a coset is in the positive cone if it has a representative $f \in C(X, \mathbf{Z})$ that is nonnegative). In many cases (minimal: GPS95; shifts of finite type: $\mathrm{BH} 96$ ), this pre-ordering is actually a partial ordering (that is, $\left.G^{+} \cap-G^{+}=\{\mathbf{0}\}\right)$ and much more. Moreover, the invariant probability measures on $(X, T)$ are in natural (affine) bijection with the normalized traces on $G$. We abbreviate the statement $g-h \in G^{+}$to $h \leq g$ when the ordering is unambigously given.

We define a trace (or a state) on a partially ordered abelian group $G$ to be a nonzero positive group homomorphism $\tau: G \rightarrow \mathbf{R}$. An element $u$ of $G^{+}$is an order unit of $G$ if for all $g \in G$, there exists a positive integer $N$ such that $-N u \leq g \leq N u$. The set of order units will be denoted $G^{++}$. A trace $\tau$ is normalized with respect to $\tau$ if $\tau(u)=1$. We may abbreviate this to normalized trace of $(G, u)$. The assignment $\mu \mapsto \tau_{\mu}$, where $\tau_{\mu}\left(\left[\chi_{U}\right]\right)=\mu(U)$ (where $U$ is a clopen subset of $X$ and $\chi_{U}$ denotes the indicator function of $U$ ), actually yields an affine homeomorphism between the Choquet simplices of invariant measures and of normalized traces on the pre-ordered abelian group $\left(G(X, T),\left[\chi_{X}\right]\right)$ with order unit.

When $T$ is minimal, GPS95] showed that the resulting pre-ordered group is not only an ordered group, but a simple dimension group. A partially ordered abelian group is simple if every nonzero element of $G^{+}$is an order unit. A partially ordered abelian group is a dimension group if it satisfies the Riesz decomposition (whenever $0 \leq a \leq b+c$, where $b, c \in G^{+}$, there exist $0 \leq b^{\prime} \leq b$ and $0 \leq c^{\prime} \leq b$ such that $a=b^{\prime}+c^{\prime}$ ) and unperforation (if $n$ is a positive integer and $g$ is an element of $G$ such that $n g \in G^{+}$, then $g \in G^{+}$). Equivalently, by [EHCLS80, a dimension group is a direct limit of simplicial groups $\mathbf{Z}^{n(\alpha)}$ ( $\alpha$ varying over a directed set) with positive homomorphisms between them. For countable dimension groups, the index set in the limit of simplicial groups can be taken to be the positive integers.

Not only did GPS95 show that when $T$ is minimal, $G(X, T)$ is a simple dimension group, but they also showed that given a countable simple dimension with order unit, $(G, u)$, there exists a minimal $T$ on $X$ such that $\left(G(X, T),\left[\chi_{X}\right]\right)$ is order isomorphic to $(G, u)$ and, moreover, $\left(G(X, T),\left[\chi_{X}\right]\right)$ is a complete invariant for strong orbit equivalence.

Simple dimension groups are completely understood (if we think that we understand Choquet simplices and countable dense subgroups of Banach spaces). Specifically, if $(G, u)$ is a partially ordered abelian group, we may construct a compact convex set $S(G, u)$ inside $\mathbf{R}^{G}$ consisting of the normalized traces. Then $(G, u)$ admits an affine representation; that is, the map ${ }^{\wedge}: G \rightarrow \operatorname{Aff} S(G, u)$, where $\widehat{g}(\tau)=\tau(g)$ (so that $\widehat{u}$ is the constant function $\mathbf{1}$ ). For details on affine representations, Choquet theory, etc., see [Alf71], Aki99], and [Goo86, Sections 5, 8, 9, 11]. As in those references, the extremal boundary of $S(G, u)$ consisting of the extreme (or pure) points will be denoted $\partial_{e} S(G, u)$.

The map ${ }^{\wedge}$ is order preserving (that is, $g \in G^{+}$entails $\widehat{g} \geq 0$ as a function). If $G$ is unperforated, then $\widehat{g}>\delta \mathbf{1}$ for some $\delta>0$ entails that $g$ is an order unit GH80. (the converse is trivial), and in fact it is sufficient that $\tau(g)>0$ for all pure $\tau$. When $G$ is a dimension group, $S(G, u)$ is a Choquet simplex Goo86, 10.17]. The representation theorem for simple dimension groups asserts that if $G$ is a noncyclic simple dimension group, then the image of $G, \widehat{G}$, is dense in $\operatorname{Aff} S(G, u)$ and $G^{+} \backslash\{0\}$ 
consists of $g$ such that $\widehat{g}$ is bounded below away from zero. Moreover, if $G$ is any torsion free abelian group and there is a group homomorphism $f: G \rightarrow$ Aff $K$ where $K$ is a Choquet simplex, and $f(G)$ is dense in Aff $K$, then equipped with the partial ordering $G^{+}:=\{0\} \cup f^{-1}\left((\text { Aff } K)^{++}\right.$) (equivalently, nonzero $g$ belongs to $G^{+}$if and only if $f(g)(k)>0$ for all $\left.k \in K\right), G$ is a simple dimension group, and if $f(u)=1$ for some $u$ in $G$, then $S(G, u)$ is affinely homeomorphic to $K$.

For nonsimple dimension groups, there are partial characterizations, but these are not usually so complete. Moreover, in the nonminimal case, $G(X, T)$ need not be partially ordered, but even if $G$ is, it need not satisfy the Riesz decomposition property (for example, all nontrivial shifts of finite type), and so need not be a dimension group.

We noted that there is a direct translation between (pure) invariant probability measures on $(X, T)$ and (pure) normalized traces on $\left(G(X, T),\left[\chi_{X}\right]\right)$. For this article, we have to translate goodness and related properties of invariant measures to properties of traces on $G(X, T)$.

Let $\mu$ be a probability measure on $X$. Define $S(\mu)=\{\mu(U) \mid U$ clopen in $X\}$. So $S(\mu)$ is a countable subset of the reals. Akin [Aki05] defined $\mu$ to be good if for all $\alpha \in S(\mu)$ and $V$ a clopen set in $X$ such that $\mu(V)>\alpha$, there exists a clopen set $U \subseteq V$ such that $\mu(U)=\alpha$. We can of course translate this directly to traces on $G(X, T)$ (assuming $\mu$ is $T$-invariant), but the direct translation is awkward to use, and instead, we obtain an equivalent formulation which is much easier to deal with. That is part of the reason for the battle of the definitions (Section 1).

For a partially ordered abelian group with order unit $(G, u)$ and $b$ in $G^{+}$, define the interval generated by $b$ to be $[0, b]:=\left\{a \in G^{+} \mid a \leq b\right\}$. The naive direct translation of goodness for a trace $\tau$ of a dimension group with order unit $(G, u)$ would be, for all $b \in[0, u], \tau([0, b])=\tau([0, u]) \cap[0, \tau(b)]$ (the last is the usual interval in $\mathbf{R})$. Our first order of business is to show this is equivalent to more usable criteria, for example, for all $b \in G^{+}, \tau([0, b])=\tau(G) \cap[0, \tau(b)]$, and it is equivalent to the condition replacing $\tau(G)$ by $\tau\left(G^{+}\right)$. Necessary and sufficient that $\tau$ be good is that there exist a sequence of elements $b_{i} \in G^{+}$such that $\widehat{b}_{i} \rightarrow 0$ (uniformly), $\tau\left(b_{i}\right) \neq 0$ and $\tau\left(\left[0, b_{i}\right]\right)=\tau\left(G^{+}\right) \cap\left[0, \tau\left(b_{i}\right)\right]$ (Corollary 1.11).

With $u=\left[\chi_{X}\right]$, and $\mu$ translating to the trace $\tau, S(\mu)$ translates to $\tau([0, u])$. These definitions, however, while useful, are still not easy to work with. There is a complete characterization of traces that are good, specifically, the image of $\operatorname{ker} \tau$ is dense (in the affine representation) in the space of affine functions that vanish at $\tau$ (a well known object in Choquet theory). It is easy to use this characterization to construct examples and nonexamples.

In addition to numerous classes of examples, we also deal with dimension group extensions (by order ideals). These correspond to closed invariant subsets of $X$, so we can ask, when is goodness preserved by the natural extension of $\mu$ ? This is answered in Proposition 4.2, extending a result from stationary systems.

If $G$ and $H$ are dimension groups, there is a natural construction of a dimension group $G \otimes H$, and pure traces of the latter are all of the form $\sigma \otimes \tau$ where $\sigma$ and $\tau$ are pure traces of $G$ and $H$ respectively. If $\sigma$ and $\tau$ are good, then so is $\sigma \otimes \tau$, but the latter can be good without either $\sigma$ or $\tau$ being good, and we investigate classes of examples, leading to a result with an algebraic geometrical flavour, Proposition 5.10 . 
In Section 7, we consider goodness en masse, that is, if $U$ is a collection of traces such that whenever $a$ and $b$ are in $G^{+}$and $\tau(b)-\tau(a)$ is bounded below away from zero as $\tau$ varies over $U$, then there exists $a^{\prime}$ in $G^{+}$with $a^{\prime} \leq b$ such that $\tau(a)=\tau\left(a^{\prime}\right)$ for all $\tau$ in $U$. In contrast to the situation with a single trace, a characterization of good sets of traces is more complicated, having to deal with special subsimplices of the trace space.

Refinability, a weakening of goodness, is discussed. We obtain a sufficient condition for a trace to be refinable (without being good) in terms of goodness en masse of a set of traces related to the trace. We use this to show that if $(R, 1)$ is a partially ordered ring with 1 as an order unit that is also a simple dimension group (examples of this are considered in Section 5), then all pure traces are refinable. This is somewhat surprising, since it is relatively easy to construct simple dimension groups of rational dimension three with exactly three pure traces (hence the trace space is a Bauer simplex), not all of which are refinable.

In examining refinability, we are led back to goodness en masse, which in turn leads to a definition of goodness for subsets of Choquet simplices that arise as intersections with closed flats. Very few of these turn out to be good, but among the good ones are faces and singleton sets (not consisting of an extreme point), and their coproducts in the category of Choquet simplices.

In Appendix A, we outline a construction of a minimal $\mathbf{Z}$-action that corresponds to the tensor product of dimension groups. In Appendix B, motivated by a result in Section 7 that the difference between refinability and goodness sometimes reduces to whether the corresponding ordered quotient group (by convex - but not directedsubgroups) is unperforated, we give some necessary and some sufficient geometrical conditions that the quotient be unperforated.

References for Choquet theory include Alfsen [Alf71, Asimow and Ellis [AE80, and various sections of Goodearl [Goo86, Sections 5, 8, 9, 11]. The latter is also good for dimension groups (and other partially ordered groups). A brief introduction to dimension groups is given in [Eff81.

\section{The Definitions Shoot it out}

Goodness and related notions deal with lifting properties, both in the original context of measures on Cantor sets, and traces on dimension groups. In order to implement the translation, we first note a property of minimal homeomorphisms, observed in Put89, Theorem 1.1]. Let $(G, u)$ be the simple dimension group corresponding to $(X, T)$ where $T$ is a minimal self-homeomorphism of the Cantor set $X$. Then $G$ can be obtained as $C(X, \mathbf{Z}) /(1-T) C(X, \mathbf{Z})$ with the quotient ordering, and $u$ is the image of the constant function 1. Putnam showed that if $0 \leq a \leq u$, then there exists a clopen set $U$ such that the image of the indicator function $\chi_{U}$ is $a$. The application of Kakutani equivalence to this result, using the fact that every nonzero element of $G$ is an order unit, yields:

(1) Suppose $B: X \rightarrow \mathbf{Z}$ is a nonnegative continuous function whose image in $G$ is denoted $b$. If $a$ is an element of $G$ such that $0 \leq a \leq b$, then there exists a continuous function $A: X \rightarrow \mathbf{Z}$ such that $0 \leq A \leq B$ (as functions) and $A \mapsto a$.

Translation of properties between those of minimal homeomorphisms and those of simple dimension groups. Property (1) permits the translation of the notion of good (and later on, goodness en masse) from measures invariant under a minimal 
homeomorphism to simple dimension groups. This is a two-way translation, so that results on traces of simple dimension groups yield their counterparts for invariant measures under minimal self-homeomorphisms of Cantor sets.

For example, Example 5 gives a simple dimension group with a continuum of pure traces (homeomorphic to the closed unit interval), such that the set of good pure traces and the set of not good pure traces are each dense in the set of pure traces. This example translates to a Cantor set with minimal self-homeomorphism $(X, T)$ for which the set of ergodic invariant measures is the unit interval, and under this identification, the good ergodic measures correspond to the algebraic numbers (over the rationals) in the unit interval having no nontrivial algebraic conjugate in the interval.

Example 3(b) similarly translates to a minimal homeomorphism of a Cantor set, with exactly three ergodic invariant measures, one of which is good, one is ugly, and one is bad, and for which the group generated by values of the measure on clopen sets is the same for each of the three ergodic measures (for a trace $\tau$ with corresponding measure, it is always true that $\tau(G)$ is the subgroup of the reals generated by $\mu(Y)$ as $Y$ varies over the clopen sets of $X)$. Example 6 yields a minimal homeomorphism with exactly two ergodic measures, both with values (on clopen sets) in $\mathbf{Z}\left[\frac{1}{2}\right]$ (in particular, they are rational-valued), neither of which is good. Example 11 translates to a minimal homeomorphism with exactly three ergodic measures, one of which is refinable but not good or bad, and one of which is not even refinable.

These examples (and others in the paper) are relatively easy to construct from the dimension group point of view, but appear to be extremely difficult to obtain via direct construction of minimal homeomorphisms. Appendix A gives a construction of minimal homeomorphisms arising from tensor products of simple dimension groups (that is, the tensor product translates, up to strong orbit equivalence, to an operation on minimal homeomorphisms), another example of this translation process. On the other hand, not every property has a translation (yet); for example, the situation in Appendix B dealing with quotients by suitable subgroups does not have an obvious translation, although it does describe a dynamical invariant.

In the nonsimple case, the situation is somewhat more complicated. Again, let $(X, T)$ be a Cantor set with homeomorphism, this time not necessarily minimal, and let $(G, u)$ be defined as in the minimal case. Kim, Roush, and S. Williams [KRW, Proposition 3.1] showed that if $(X, T)$ is an irreducible shift of finite type, then single projections lift, as in the minimal case; that is, if $0 \leq a \leq u$, then there exists clopen $U$ such that $\chi_{U} \mapsto a$. By applying inverse limits and Kakutani equivalence, [BH3] showed that this extends, with provisos, to all $(X, T)$ :

(2) Suppose $B: X \rightarrow \mathbf{Z}$ is a nonnegative continuous function whose image in $G, b$, is an order unit of $G$. If $a$ is an element of $G$ such that $0 \leq a \leq b$, then there exists a continuous function $A: X \rightarrow \mathbf{Z}$ such that $0 \leq A \leq B$ (as functions) and $A \mapsto a$.

A minor proviso is that the pre-ordering on the quotient need not be a partial ordering, although it is in most cases of interest; the major proviso restricts the larger element to be an order unit. This is enough, however, for the notion of order unit good to translate directly in the nonminimal case.

We are restricting ourselves throughout to the case(s) that $G$ obtained from $(X, T)$ be a dimension group (or more generally, we are considering goodness and 
related notions for dimension groups). If $(X, T)$ is a shift of finite type arising from a primitive matrix, the corresponding $G$ is not a dimension group, and in fact, the full lifting result (1) (that is, without $b$ being an order unit) does not hold. However, it is plausible that when $G=C(X, \mathbf{Z}) /(1-T) C(X, \mathbf{Z})$ is a dimension group, then the full lifting result does hold (in fact, it is likely that one-way results still apply without assuming $G$ is a dimension group). If this were so, the translation of goodness from traces on dimension groups to invariant measures of $(X, T)$ would be two-way.

Since most of our attention is devoted to simple dimension groups - all of which come out of minimal homeomorphisms - we don't regard the apparently incomplete translation in the nonminimal case to be a serious problem, especially since order unit goodness does translate in general.

Let $G$ be a dimension group, and let $\tau$ be a trace thereon. We say $\tau$ is good if for all $a, b \in G^{+} \backslash\{0\}$ such that $\tau(a)<\tau(b)$, there exists $c$ in $G^{+}$such that $c \leq b$ and $\tau(c)=\tau(a)$.

Let $b$ be a positive element of $G$. Denote by $[0, b]$, the interval generated by $b$, $\left\{c \in G^{+} \mid c \leq b\right\}$. Then an alternative formulation of the definition of a good trace is simply that for all $b$ in $G^{+}, \tau([0, b])=\tau\left(G^{+}\right) \cap[0, \tau(b)]$ (the second interval is the usual one in $\mathbf{R}$ ). This implies a stronger property, with the plus superscript deleted (Lemma 1.1 below). The direct translation of the definition of good measure would be, for fixed order unit $u$, and any $b \leq u$, that $\tau([0, b])=\tau([0, u]) \cap[0, \tau(b)]$. We show in Lemmas 1.1 and 1.3 that this is equivalent to our definition of good.

Let $\tau$ be a trace of $G$. We say an element $b$ of $G^{+}$is weakly $\tau$-good or weakly good with respect to $\tau$ if for every $a$ in $G^{+}$such that $0<\tau(a)<\tau(b)$, there exists $c$ in $[0, b]$ such that $\tau(c)=\tau(a)$. The element $b$ in $G^{+}$is $\tau$-good or good with respect to $\tau$ if the $a$ can vary over $G$, that is, $\tau([0, b])=\tau(G) \cap[0, \tau(b)]$. Obviously, 0 is always $\tau$-good, and $\tau$ is good if and only if every element of $G^{+}$is weakly $\tau$-good. The next result says that this is equivalent to all elements of the positive cone being good with respect to $\tau$.

If every order unit is $\tau$-good, then we say that $\tau$ is order unit good. Because of (2), order unit goodness admits a two-way translation with the corresponding concept for nonminimal homeomorphisms (at least when $G=C(X, \mathbf{Z}) /(\mathrm{I}-T)(C(X, \mathbf{Z})$ ) is a dimension group). Translating to measures, order unit good amounts to restricting the clopen set $B$ (in the definition of good measure) to those for which $\bigcup T^{n} B=X$. All results about order unit good traces translate to results about the corresponding invariant measures, and vice versa. In particular, all the examples given in this paper can be reformulated in terms of self-homeomorphisms (usually minimal) of Cantor sets. Order unit good traces (and thus their counterpart measures) admit a particularly effective characterization, Proposition 1.7.

\section{Lemma 1.1.}

(a) If $\tau$ is a normalized trace of $(G, u)$ such that for all $b$ in $[0, u]$ and $a \in[0, u]$ such that $\tau(a)<\tau(b)$, there exists $c \in[0, b]$ such that $\tau(c)=\tau(a)$, then $\tau$ is good.

(b) If $\tau$ is a good trace of $G$, then $\tau([0, b])=\tau(G) \cap[0, \tau(b)]$ for all b in $G^{+}$; that is, every element of $G^{+}$is weakly good implies every element of $G^{+}$is good with respect to $\tau$.

Proof. (a) Select $a \in G^{+}$such that $\tau(a)<\tau(b)$. There exists an integer $n$ such that $a \leq n u$ (from the definition of order unit). By Riesz decomposition, there exist 
$a_{i} \in[0, u](i=1,2, \ldots, n)$ such that $a=\sum a_{i}$. Since $a_{n} \leq a$, we have $\tau\left(a_{n}\right)<\tau(b)$, so there exists $c_{n} \in[0, b]$ with $\tau\left(c_{n}\right)=\tau\left(a_{n}\right)$. Then $\tau\left(\sum_{i<n} a_{i}\right)<\tau\left(b-c_{n}\right)$, and we proceed by induction on $n$.

(b) Given $a$ in $G$ and $b$ in $G^{+}$such that $0<\tau(a)<\tau(b)$ (if either equality holds, the result is immediate), there exist positive elements $c$ and $d$ such that $a=c-d$ (since $G$ is directed, part of the definition of a partially ordered abelian group). Since $\tau(c)-\tau(d)=\tau(a)>0$, we can apply weak goodness to $d$ and $c$, yielding an element $e$ in $[0, c]$ such that $\tau(e)=\tau(d)$. Then $c-e$ is a positive element of $G$ and $\tau(c-e)=\tau(a)$, so we can apply weak goodness to the pair $c-e$ and $b$.

If $\tau$ is a trace on $(G, u)$, we say that $\tau$ is reasonable if for all $b$ in $G^{+}, \tau([0, b])$ is dense in $\tau(G) \cap[0, \tau(b)]$. Most traces that we are likely to encounter are reasonable, but some are not: let $(H, v)$ be any nonzero dimension group with order unit, take $G=\mathbf{Z} \oplus H$ with the coordinatewise ordering, and let $\sigma$ be a normalized trace of $(H, v)$. Then $\tau:(m, h) \mapsto m+\sigma(h)$ is not reasonable if $|\sigma([0, v])| \geq 3$ (take $b=(1,0))$. If $\sigma$ has dense range in $\mathbf{R}$, then $\tau$ does as well, but $\tau([0, b])$ is discrete. More generally, if $G$ has two simple quotients (by order ideals), at least one of which is cyclic, then $G$ admits a trace that is not reasonable. By Lemma 1.1, a good trace is reasonable.

The terms pure, ergodic, and extremal, when applied either to traces on a dimension group or invariant measures on $(X, T)$, are synonymous and mean not expressible as a proper convex linear combination of other normalized traces/invariant measures. (Other terms meaning the same thing are occasionally seen, usually in translation from Russian. These include minimal, irreducible, and indecomposable; we will not use these in this context.) In the literature, ergodic has usually been employed in the dynamical situation, while pure and extremal appear with respect to dimension groups and Choquet theory.

A trace is discrete Goo86 if its image is cyclic. A dimension group $(G, u)$ with order unit has no discrete (pure) traces if and only if its image in $\operatorname{Aff} S(G, u)$ is dense, with respect to the supremum norm GH80, Theorem 4.8], and then is called approximately divisible.

We recall the purity criterion for traces on dimension groups with order unit, $(G, u)$, given in [GH80, Theorem 3.1]:

the trace $\tau$ is pure if and only if for all $\epsilon>0$ and all $a$ and $b$ in $G^{+}$,

there exists $c \in[0, a] \cap[0, b]$ (that is, $c \leq a ; c \leq b$; and $c \in G^{+}$) such that $\tau(c)>\min \{\tau(a), \tau(b)\}-\epsilon$.

Lemma 1.2. Let $(G, u)$ be a dimension group with order unit.

(a) Suppose all order ideals of $G$ admitting an order unit are approximately divisible. Then all traces of all order ideals with order unit (including $(G, u)$ itself) are reasonable and have dense range in $\mathbf{R}$.

(b) If $\tau$ is a pure trace of $G$, then $\tau$ is reasonable.

Proof. (a) It suffices to do this when $b$ is an order unit, since we can take the order ideal generated by $b$. Then $G$ has dense image in Aff $S(G, b)$ (so $\widehat{b}=\mathbf{1}$, the constant function). Hence for each $n$, there exists $b_{n}$ such that $n^{-1} \mathbf{1}>\widehat{b}_{n}>(n+1)^{-1} \mathbf{1}$ (approximate the constant affine function with value $(1 / 2)\left(n^{-1}+(n+1)^{-1}\right)$ with an error of less than $1 / 2 n(n+1))$. Then $k b_{n}$ belongs to $[0, b]$ for $1 \leq k \leq n$, and the value of $k b_{n}$ at every normalized trace belongs to the interval $(k /(n+1), k / n)$, and thus the set of values of $\left\{k b_{n}\right\}_{1 \leq k \leq n ; n=1,2, \ldots}$ is dense in $[0,1]=[0, \tau(b)]$. 
(b) For fixed $b$ in $G^{+}$, suppose $c$ is an element of $G$ such that $0<\tau(c)<\tau(b)$. Then we can write $c=g-h$ where $g$ and $h$ are positive elements. Apply the purity criterion to the pair $g, h$; given $\epsilon$, we may find positive $f \leq g, h$ such that $\tau(f)>\min \{\tau(g), \tau(h)\}-\epsilon=\tau(h)-\epsilon$. Then $c=(g-f)-(h-f)$, and $\tau(h-f)<\epsilon$. If $\epsilon<\tau(b-c) / 2$, then $\tau(g-f)<\tau(b)$. Since $g-f \geq 0$, we may apply the purity criterion again, obtaining $0 \leq e \leq g-f, b$ such that $\tau(e)>\tau(g-f)-\epsilon$, and thus $|\tau(e)-\tau(c)|<|\tau(e-g+f)|+|\tau(g-f-c)|<2 \epsilon$, and $e \in[0, b]$.

Suppose $H$ is a subset of $G^{+}$such that every element of $G^{+}$is a sum of elements of $H$; we call $H$ a generating subset of $G$. Examples include $H=[0, u]$ if $u$ is an order unit of $G$, and $H=\left\{h_{1} \otimes h_{2} \mid h_{i} \in H_{i}\right\}$ if $G=G_{1} \otimes G_{2}$ (the ordered tensor product; this is a dimension group if both $G_{i}$ are, and will be discussed later), and $H_{i}$ is a generating subset of $G_{i}$.

Lemma 1.3. Let $G$ be a dimension group and let $\tau$ be a reasonable trace such that $\tau(G)$ is dense in $\mathbf{R}$. Let $v_{1}$ and $v_{2}$ be nonzero elements of $G^{+}$. If both are (weakly) $\tau$-good, then $v_{1}+v_{2}$ is as well.

Proof. First suppose that both $v_{i}$ are $\tau$-good; we wish to show $v_{1}+v_{2}$ is as well. Select $f$ in $G$ such that $0<\tau(f)<\tau\left(v_{1}+v_{2}\right)$ (if either inequality is replaced by equality, there is nothing to do). If $\tau(f)<\max \left\{\tau\left(v_{1}\right), \tau\left(v_{2}\right)\right\}$, then the result follows immediately from goodness of $v_{i}$.

So we may assume that $\tau(f)>\max \left\{\tau\left(v_{i}\right)\right\}$. Since $\tau(f) \in\left[0, \tau\left(v_{1}\right)+\tau\left(v_{2}\right)\right]=$ $\left[0, \tau\left(v_{1}\right)\right]+\left[0, \tau\left(v_{2}\right)\right]$, there exist $s_{i}$ in $\left[0, \tau\left(v_{i}\right)\right]$ such that $\tau(f)=s_{1}+s_{2}$. Then the larger one of them, say $s_{1}$ (after relabelling), must exceed $\tau(f) / 2$, and the other one must be nonzero. By reasonableness of $\tau$, given $\epsilon>0$, there exists $h$ in $\left[0, v_{1}\right]$ such that $\left|s_{1}-\tau(h)\right|<\epsilon$, where we choose $\epsilon<\min \left\{\tau\left(v_{2}-s_{2}\right), s_{2}\right\}$. Then $\left|\tau(f-h)-s_{2}\right|=\left|\tau(h)-s_{1}\right|<\epsilon$, so that $\tau(f-h)>s_{2}-\epsilon>0$ and $\tau(f-h)<s_{2}+\epsilon<\tau\left(v_{2}\right)$. By goodness of $v_{2}$ applied to $f-h$, there exists $h^{\prime}$ in $\left[0, v_{2}\right]$ such that $\tau(f-h)=\tau\left(h^{\prime}\right)$. Setting $c=h+h^{\prime}$, we see that $c$ belongs to $\left[0, v_{1}+v_{2}\right]$ and $\tau(c)=\tau(f)$.

Now suppose the $v_{i}$ are $\tau$-weakly good. Suppose $a \in G^{+}$and $0<\tau(a)<$ $\tau\left(v_{1}+v_{2}\right)$. Set $\alpha_{i}=\tau\left(v_{i}\right) \tau(a) / \tau\left(v_{1}+v_{2}\right)$, so that $0<\alpha_{i}<\tau\left(v_{i}\right)$ and $\tau(a)=\alpha_{1}+\alpha_{2}$. Choose $\epsilon<\min \left\{\tau\left(v_{1}\right)-\alpha_{1}, \tau\left(v_{2}\right)-\alpha_{2}, \alpha_{1}\right\}$. By reasonableness of $\tau$, we may find $d_{1} \in[0, a]$ such that $\alpha_{1}-\epsilon<\tau\left(d_{1}\right)<\alpha_{1}<\tau\left(v_{1}\right)$. Then $0<\tau\left(a-d_{1}\right)<\alpha_{2}+\epsilon<$ $\tau\left(v_{2}\right)$. By weak goodness of the $v_{i}$, there exist $c_{1} \in\left[0, v_{1}\right]$ such that $\tau\left(c_{1}\right)=\tau\left(d_{1}\right)$ and $c_{2} \in\left[0, v_{2}\right]$ such that $\tau\left(a-d_{1}\right)=\tau\left(c_{2}\right)$. Then $c_{1}+c_{2} \in\left[0, v_{1}\right]+\left[0, v_{2}\right] \subseteq$ $\left[0, v_{1}+v_{2}\right]$ and $\tau\left(c_{1}+c_{2}\right)=\tau(a)$.

Corollary 1.4. Suppose that $G$ is a dimension group, and $\tau$ is a reasonable trace with dense range in $\mathbf{R}$. If $G^{+}$admits a generating set consisting of weakly $\tau$-good elements, then $\tau$ is good.

This will be superseded (in the simple case) by Corollary 1.11, where it is shown that sufficient for goodness is that there exist a sequence of nonzero weakly $\tau$-good elements $v_{i}$ such that $\widehat{v}_{i} \rightarrow 0$ (that is, with respect to the supremum norm on $S(G, u))$.

Suppose that $b$ is an element of the positive cone of the dimension group $G$. Recall that $[0, v]$ denotes $\left\{g \in G^{+} \mid g \leq v\right\}$. Then $\tau$ is group-like with respect to $v$ if whenever $a$ belongs to $G$ and $0 \leq \tau(a) \leq \tau(v)$, there exists $c$ in $[0, v]$ such that 
$\tau(c)=\tau(a)$; that is, $\tau([0, v])=\tau(G) \cap[0, \tau(v)]$. We say $\tau$ is weakly group-like with respect to $v$ if $a$ is restricted to $G^{+}$; that is, $\tau([0, v])=\tau\left(G^{+}\right) \cap[0, \tau(v)]$.

Examples. There exist simple dimension groups (which are free abelian groups of rank 3 or 4) with two pure traces such that neither one is good, or exactly one is good, or both are good. There also exist simple dimension groups with two pure traces such that neither trace is good but become good on tensoring with the rationals, and also that do not become good on tensoring. Similarly, there exist simple dimension groups for which being group-like depends on the choice of order unit.

Many of the examples are of the form $G \subset \mathbf{R}^{n}$, where $G$ is a dense subgroup of rank $n+1$ (or more), equipped with the strict ordering inherited from $\mathbf{R}^{n}$; that is, $G^{+} \backslash\{0\}=\left(\mathbf{R}^{n}\right)^{++} \cap G$. These are dimension groups, and the $n$ projection maps (onto the first, second, ... coordinates, respectively), $\tau_{1}, \tau_{2}, \ldots$, are the only pure traces. If $G$ is the abelian group spanned by three vectors, $v_{1}, v_{2}, v_{3}$ of $\mathbf{R}^{2}$, then $G$ is dense if and only if the set of three $2 \times 2$ determinants, $\left\{v_{1} \wedge v_{2}, v_{1} \wedge v_{3}, v_{2} \wedge v_{3}\right\}$, is linearly independent over the rationals.

Example 1. Let $\{1, \alpha, \beta\}$ be a set of real numbers that is linearly independent over the rationals and such that $0<\alpha<1<\beta$; set $G=\langle(1,0),(0,1),(\alpha, \beta)\rangle$. Linear independence of $\{1, \alpha, \beta\}$ guarantees $G$ is dense in $\mathbf{R}^{2}$, and thus is a simple dimension group with respect to the strict order. Let $\tau=\tau_{2}$ be the projection onto the second coordinate.

(a) $\tau$ is not good. Set $a=(1,1)$ and $b=(\alpha, \beta)$; we show the existence of $c$ in $G^{+}$such that $\tau(a)=\tau(c)$ and $c \leq b$ is impossible. As a group homomorphism $\tau: G \rightarrow \mathbf{R}$, its kernel is spanned by $(1,0)$. Hence $c-a=(m, 0)$ for some integer $m$, that is, $c=(m+1,1)$; since $c$ is in $G^{+} \backslash 0$, we must have $m \geq 0$, whence $\tau_{1}(c) \geq 1$. But $\tau_{1}(b)=\alpha<1 \leq \tau_{1}(c)$, contradicting $c \leq b$.

(b) The extension of $\tau$ to $G \otimes \mathbf{Q}$ is good. Write $a=\left(a_{1}, a_{2}\right)$ and $b=\left(b_{1}, b_{2}\right)$ in $(G \otimes \mathbf{Q})^{+}$with $0<a_{2}<b_{2}$ (so $\left.\tau(a)<\tau(b)\right)$. Since $a$ and $b$ are nonzero positive elements, $a_{1}, b_{1}>0$. Since the positive rationals are dense in the positive reals, there exists a positive rational number $q$ such that $0<a_{1}-q<b_{1}$. Set $c=a-q \cdot(1,0)=\left(a_{1}-q, a_{2}\right)$; then $c$ belongs to $G \otimes \mathbf{Q}$, is strictly positive (hence is in the positive cone), $\tau(c)=a_{2}=\tau(a)$, and $c$ is strictly less than $b$, so $b-c \in(G \otimes \mathbf{Q})^{+}$, verifying goodness.

(c) With respect to the order unit $u=(1,1), \tau$ is group-like. Suppose $a=(r, s)$ is an element of $G^{+}$such that $\tau(a) \leq 1$, that is, $s \leq 1$. If $s=1$, set $b=u$ and we are done. We may thus suppose $0<s<1$. Decompose $r=\lfloor r\rfloor+\{r\}$ into its integer and fractional parts. If $\{r\}$ is not zero (that is, $r$ is not an integer), then set $b=a-\lfloor r\rfloor(1,0)=(\{r\}, s)$, so that $b$ belongs to $[0, u]$ and $\tau(b)=s$.

Otherwise, $r$ is an integer. Then we can write $a=(r, s)=m(1,0)+n(0,1)+$ $p \cdot(\alpha, \beta)$, where $m, n, p$ are integers. Evaluating at the first coordinate yields $r=$ $m+p \alpha$; since $\{1, \alpha\}$ is linearly independent over $\mathbf{Q}$ and $r$ is an integer, we deduce $r=m$ and $p=0$. Hence $a=(m, n)$, which is impossible, since the second coordinate of $a$ is strictly between 0 and 1 .

(d) With respect to the order unit $u=(\alpha, \beta), \tau$ is not group-like. With $a=(1,1)$, this follows from the argument in (a).

Example 2. Let $\alpha_{i}, \beta_{i}(i=1,2)$ be four positive real numbers such that each set $\left\{1, \beta_{1}, \beta_{2}\right\}$ and $\left\{\beta_{1}-\alpha_{1}, \beta_{2}-\alpha_{2}, \alpha_{1} \beta_{2}-\beta_{2} \alpha_{1}\right\}$ is linearly independent over the 
rationals (this will occur, for example, if $\left\{1, \alpha_{i}, \beta_{i}\right\}$ is algebraically independent over the rationals). Assume in addition that $\alpha_{i}<\beta_{i}$ and $\alpha_{1}+\alpha_{2}<1<\beta_{1}+\beta_{2}$. Set $G=\left\langle(1,1),\left(\alpha_{1}, \beta_{1}\right),\left(\alpha_{2}, \beta_{2}\right)\right\rangle$.

Linear independence of the second set guarantees $G$ is dense in $\mathbf{R}^{2}$, so equipped with the strict ordering, $G$ is a simple dimension group. The important property here is that $\operatorname{ker} \tau$ is trivial, as follows from linear independence of $\left\{1, \beta_{1}, \beta_{2}\right\}$.

For no order unit $u$ is $\tau=\tau_{2}$ group-like. This is a special case of the following easy result, which is preliminary to our later characterization of good pure traces on simple dimension groups.

The group of infinitesimals is denoted $\operatorname{Inf}(G)$, and is equal to the intersection of the kernels of all traces (when $G$ has an order unit).

Proposition 1.5. Suppose that $(G, u)$ is a dimension group, and $\tau$ is a trace of $G$ such that $\operatorname{ker} \tau=\operatorname{Inf}(G)$. If $G$ has more than one trace, then no positive element $b$ such that $\tau(b) \neq 0$ is weakly $\tau$-good (in particular, every element is not $\tau$-good).

Proof. Without loss of generality, $\operatorname{Inf}(G)=0$ and $\tau(u)=1$. Suppose there exists another normalized trace unequal to $\tau, \sigma$. If there exist $a, b$ in $G^{+}$such that $\tau(a)<\tau(b)$ but $\sigma(a)>\sigma(b)$, and $0 \leq c \leq b$ such that $\tau(c)=\tau(a)$, then $a=c$, and thus $\sigma(c)>\sigma(b)$, a contradiction. Hence if $b$ is weakly $\tau$-good, then $\tau(a)<\tau(b)$ implies $\sigma(a) \leq \sigma(b)$ for all other traces $\sigma$. Multiply $a$ and $b$ by a big enough integer $K$ such that $\tau(K a)<\tau((K-1) b)$. Then $\sigma(K a) \leq \sigma(K-1) b$ for all traces unequal to $\tau$; thus $\tau(a)<\tau(b)$ entails $\sigma(a)<\sigma(b)$ for all traces, and thus $b-a$ is an order unit. However, since the elements of $G^{+}$separate traces, for any pair of distinct normalized traces there exist $a$ in $G^{+}$such that $\tau(a)<\tau(b)$ and $\sigma(a)>\sigma(b)$.

If $\tau$ is a trace on a dimension group having more than one pure trace, and $\operatorname{ker} \tau=\operatorname{Inf} G$ (e.g., if $\operatorname{ker} \tau=\{0\}$ ), then $\tau$ is a bad trace. A consequence of the proposition is that a bad trace is not good.

Lemma 1.6. If $G$ is a simple dimension group, $\tau$ is pure, $G$ has more than one trace, and $\operatorname{ker} \tau / \operatorname{Inf}(G)$ is cyclic, then $\tau$ fails to be good.

Proof. Since $G / \operatorname{Inf}(G)$ is a simple dimension group with the same traces and values, we may assume that $\operatorname{Inf}(G)=0$. If $\operatorname{ker} \tau=\{0\}$, we are in the case of Proposition 1.5 (since good implies group-like with respect to every order unit). Otherwise, we note that $\operatorname{ker} \tau$ is obviously torsion-free, so must be isomorphic to $\mathbf{Z}$; i.e., there exists $g$ in $G$ such that $\operatorname{ker} \tau=\mathbf{Z} g$. Since $\operatorname{Inf}(G)=0$, there exists a pure trace $\tau_{1}$ such that $\tau_{1}(g) \neq 0$; by replacing $g$ by $-g$ if necessary, we may assume $\tau_{1}(g)>0$. Select any nonzero positive element $u$ such that $\tau_{1}(u)<\tau_{1}(g)$ (such exist from elementary results); then $u$ is an order unit for $G$ from simplicity. Renormalize the traces at $u$ (so $\tau_{1}(g)>\tau_{1}(u)=\tau(u)=1$ ), and represent $G$ in the group of affine functions on its normalized trace space.

By density of the positive cone in the positive cone of the latter, there exists $a$ in $G^{+}$such that $\tau(a)<1 / 2$ and $1<\tau_{1}(a)<\tau_{1}(g)$. If $b$ were any element of $G^{+}$such that $\tau(a)=\tau(b)$, then $b=a+m g$ for some integer $m$. Applying $\tau_{1}$, we have $\tau_{1}(b)=\tau_{1}(a)+m \tau_{1}(g)$. If $m$ were negative, we would obtain $\tau_{1}(b)<0$, a contradiction. If $m \geq 0$, then $\tau_{1}(b) \geq \tau_{1}(a)>1$; hence $b$ cannot be in $[0, u]$. Hence $\tau$ is not group-like with respect to some order unit, and therefore $\tau$ is not good.

Both of these easy results will be superseded, but they are worth mentioning, as the criteria are usually easy to verify. 
We say a dimension group $G$ with an order unit is approximately divisible if for all pure traces $\sigma, \sigma(G)$ is nondiscrete in $\mathbf{R}$. This is equivalent to the natural map $G \rightarrow \operatorname{Aff}(S(G, u))$ having norm-dense range, and also to the image of $G^{+}$being dense in the positive cone of $\operatorname{Aff}(S(G, u))$ [GH80, Theorem 4.8]; if $G=\mathrm{K}_{0}(A)$ for some unital AF algebra, approximate divisibility of $G$ is equivalent to $A$ having no finite-dimensional representations.

Fix an order unit $u$ of $G$ and a pure trace $\tau$, which we may now assume is normalized with respect to $u$. Form the affine representation $G \rightarrow \operatorname{Aff}(S(G, u)), g \mapsto$ $\widehat{g}$, where $\widehat{g}(\sigma)=\sigma(g)$ for normalized traces $\sigma$. With respect to the usual ordering, $\operatorname{Aff}(S(G, u))$ is itself a dimension group, and $\tau$ induces a map $\tilde{\tau}: \operatorname{Aff}(S(G, u)) \rightarrow \mathbf{R}$ with kernel denoted $\{\tau\}^{\perp}=\{f \in \operatorname{Aff} S(G, u) \mid f(\tau)=0\}$. Since $\tau$ is pure, $\{\tau\}^{\perp}$ is an order ideal of $\operatorname{Aff}(S(G, u))$, in fact, a maximal order ideal.

If $\tau$ is not known to be pure, it still makes sense to consider

$$
\{\tau\}^{\vdash}:=\{h \in \operatorname{Aff} S(G, u) \mid h(\tau)=0\}
$$

(note the slight difference in notation: when $\tau$ is pure, we use the perp symbol ${ }^{\perp}$, which signals that $\tau^{\perp}$ is an order ideal in Aff $S(G, u)$; but in general, when $\tau$ need not be pure, we use ${ }^{\vdash}$ ). The set $\tau^{\vdash}$ is a closed convex codimension one subspace of Aff $S(G, u)$, not (necessarily) an order ideal. Obviously ${ }^{\wedge}$ sends $\operatorname{ker} \tau$ to $\{\tau\}^{\vdash}$.

Recall the definition of order unit good (for a trace $\tau$ ), obtained by restricting the definition of good to require that $b$ be an order unit; that is, $\tau([0, b])=\tau(G) \cap[0, \tau(b)]$ for all order units $b$. Obviously if $G$ is simple and $\tau$ is order unit good, then it is good (since all nonzero elements of $G^{+}$are order units).

Proposition 1.7. Let $G$ be an approximately divisible dimension group with order unit, and let $\tau$ be a trace. Then $\tau$ is order unit good if and only if the image of $\operatorname{ker} \tau$ is norm dense in $\{\tau\}^{\vdash}$.

Proof. Fix an order unit, $u$, with respect to which the trace space, $S(G, u)$, and the corresponding affine representation, ${ }^{\widehat{ }}$, are determined. We may assume that $\tau(u)=1$.

Suppose that $\tau$ is order unit good. Select $h$ in $\{\tau\}^{\vdash}$. From approximate divisibility of $G$, given $\epsilon>0$, there exists $g$ in $G$ such that $\|\widehat{g}-h\|<\epsilon$, so that $|\tau(g)|<\epsilon$. Again by density of $\widehat{g}$, there exists $g_{0}$ approximating to within $\epsilon$ the constant function with value $2 \epsilon$, so that $\left\|\widehat{g}_{0}\right\|<3 \epsilon$, and thus $g^{\prime}:=g_{0}+g$ satisfies $\left\|\widehat{g}^{\prime}-h\right\|<4 \epsilon$ and $\tau\left(g^{\prime}\right)>0$.

Yet again by density of $\widehat{g}$ in the affine representation, there exists $b$ in $G$ such that $4 \epsilon<\widehat{b}(\sigma)<5 \epsilon$ for all $\sigma$ in $S(G, u)$ (for example, a sufficiently fine approximant of the constant affine function with value $9 \epsilon / 2)$. Automatically, $b$ is an order unit, thus positive, and $\tau\left(g^{\prime}\right)<\tau(b)$. By order unit goodness, there exists $0 \leq c \leq b$ such that $\tau(c)=\tau\left(g^{\prime}\right)$; in particular, $\|\widehat{c}\| \leq\|\widehat{b}\|<5 \epsilon$. Thus $y:=g^{\prime}-c$ belongs to $\operatorname{ker} \tau$, and $\|h-\widehat{y}\| \leq\left\|h-\widehat{g}^{\prime}\right\|+\|\widehat{c}\|<9 \epsilon$.

Now suppose that the image of $\operatorname{ker} \tau$ is dense in $\{\tau\}^{\vdash}$. Fix an order unit $u$ (for constructing the affine representation), let $b$ be an order unit and let $a$ be an element of $G^{+}$such that $0<\tau(a)<\tau(b)$. Set $h=\tau(a) \widehat{b} / \tau(b)$, so that $h$ is strictly positive and $(h-\widehat{a})(\tau)=0$; hence $h-\widehat{a} \in\{\tau\}^{\vdash}$. Select $\epsilon$ so that

$$
0<\epsilon<\left(\inf _{\sigma \in S(G, u)}\{h(\sigma)\}\right) \cdot \min \left\{\frac{\tau(b)-\tau(a)}{\tau(a)}, 1\right\} ;
$$


we may find $g$ in $\operatorname{ker} \tau$ such that $\|\widehat{g}-h+\widehat{a}\|<\epsilon$. Now consider $c=g+a$. Its value at $\tau$ is $\tau(a)$, and for every normalized trace $\sigma$, we have $|\sigma(g+a)-\sigma(h)|<\epsilon$, so $\sigma(g+a)>\sigma(h)-\epsilon>0$; thus $g+a$ is an order unit, so in particular, $c$ is positive. Next $\sigma(b)-\sigma(g+a)=(\widehat{b}-h)(\sigma)+(h-\widehat{g}-\widehat{a})(\sigma)$, and this is at least $\sigma(b)(1-\tau(a) / \tau(b))-\epsilon=h(\sigma) \tau(b) / \tau(a)(1-\tau(a) / \tau(b))-\epsilon>0$. Hence $\widehat{b}-\widehat{g}-\widehat{a}$ is strictly positive, and thus $b \geq g+a=c$.

Corollary 1.8. If $G$ is a simple dimension group other than $\mathbf{Z}$ and $\tau$ is a trace, then $\tau$ is good if and only if for any (hence for every) order unit, the image of ker $\tau$ is norm dense in $\{\tau\}^{\vdash}$.

This even applies when $\tau$ is the unique (up to scalar multiple) trace, since in that case $\{\tau\}^{\perp}=\{0\}$. It is a trivial application of unperforation that uniqueness of the trace implies goodness. A minor modification of the arguments above yield semi-characterizations of weakly good elements (Lemma 1.10 and Corollary 1.11). In examples (to follow), we usually deal with pure traces, because it is easier to calculate $\operatorname{ker} \tau$ and $\{\tau\}^{\perp}$.

An order preserving group homomorphism between dimension groups with order unit $\phi:(G, u) \rightarrow(H, v)$ (such that $\phi(u)=v)$ is called an extended homomorphism (or extended) if for all pure normalized traces $\tau$ of $(G, u)$, there exists a unique (normalized) trace $\sigma$ of $(H, v)$ such that $\tau=\sigma \circ \phi$. (Uniqueness of $\sigma$ guarantees it is pure, as the pre-image of an extreme point is a closed face.)

This entails that $\phi^{-1}\left(H^{++}\right)=G^{++}$(that is, for $g$ in $G$, if $\phi(g)$ is an order unit of $H$, then $g$ is an order unit of $G$ ), but does not imply $\phi$ is an order-embedding. For example, if $G$ is simple and $\operatorname{Inf}(G) \neq 0$, then the quotient $H=G / \operatorname{Inf}(H)$ is a simple dimension group (for general dimension groups, it is not true that if $G$ is a dimension group, then $G / \operatorname{Inf}(G)$ is as well), and the quotient map is extended. (However, if $G$ and $H$ are simple dimension groups and $\operatorname{Inf}(G)=0$, then an extended map from $G$ to $H$ is an order embedding.)

Examples arise when $G$ is a simple and noncyclic dimension group, and $H$ is a dense subgroup of $\operatorname{Aff}(S(G, u))$ containing $\widehat{G}$ (and $H$ is equipped with the strict ordering); then $\phi$ is just the map ${ }^{\wedge}$ followed by the inclusion. When $G$ and $H$ are simple, modulo infinitesimals and order automorphisms, this is the only type of extended map between them.

In general, extended maps induce a natural affine homeomorphism $S(G, u) \leftarrow$ $S(H, v)$ given by $\sigma \mapsto \sigma \circ \phi$. This preserves the extremal boundaries (the sets of extreme points), and induces an isometric isomorphism between their affine function spaces. The following corollary is somewhat unexpected. For example, if $G$ is a dense subgroup of Aff $(K)$ where $K$ is a Choquet simplex, then we can take $H$ to be any intermediate group, $G \subseteq H \subseteq \operatorname{Aff}(K)$ (both $G$ and $H$ are equipped with the strict ordering, so they are simple dimension groups), and there is no obvious reason why goodness of $\sigma \mid G$ should imply that of $\sigma \mid H$ when $\sigma$ is an extreme point of $K$. However, this is a consequence of the next result, since $G \subseteq H$ is an extended map.

Corollary 1.9. Let $\phi:(G, u) \rightarrow(H, v)$ be an extended homomorphism of dimension groups. Suppose $\tau$ is a (normalized) trace of $(G, u)$ that is order unit good, and $\sigma$ is the (normalized) trace of $(H, v)$ such that $\tau=\sigma \circ \phi$. Then $\sigma$ is order unit good. 
Proof. It suffices to show ker $\sigma$ has norm-dense image in $\{\sigma\}^{\vdash}$. However, we note that $\phi(\operatorname{ker} \tau) \subseteq \operatorname{ker} \sigma$, and from Proposition 1.7, $\operatorname{ker} \tau$ has dense range in $\{\tau\}^{\vdash}$. It is trivial that $\phi$ induces an isometric isomorphism $\{\tau\}^{\vdash} \rightarrow\{\sigma\}^{\vdash}$, so that $\phi(\operatorname{ker} \tau)$ has dense image in $\{\sigma\}^{\vdash}$, and thus so does $\operatorname{ker} \sigma$.

Obviously we cannot say anything when $\tau$ is not good (e.g., if $H$ is a real vector space, then all traces of $H$, even impure ones, are good). We will see in Example 7(b) that extended maps do not generally preserve goodness; of course this phenomenon can only occur in the nonsimple case.

Here is a situation where there appears to be a difference between pure and impure traces, although not very much.

Lemma 1.10. Let $(G, u)$ be an approximately divisible dimension group with order unit, let $\tau$ be a trace thereon, and let $v$ in $G^{+}$be weakly $\tau$-good such that $\tau(v)>0$.

(i) If $\tau$ is pure, then for all $\epsilon$, for all $h$ in $\left(\{\tau\}^{\perp}\right)^{+}$, there exists $g$ (depending on $h$ ) in $\operatorname{ker} \tau$ such that $\|\widehat{g}-h\|<\epsilon+\|\widehat{v}\|$ (the norm is computed with respect to the order unit $u$ ).

(ii) If $v$ is $\tau$-good, then the same conclusion holds for all $h$ in $\{\tau\}^{\vdash}$.

Remark. The difference is that a stronger assumption is made on $v$ in the impure case than in the pure case.

Proof. (i) There exists $g_{0}$ in $G^{+}$such that $\left\|\widehat{g}_{0}-h\right\|<\epsilon$, by approximate divisibility. Without loss of generality, $\epsilon<\tau(v)$, and thus $0 \leq \tau\left(g_{0}\right)<\epsilon<\tau(v)$. By weak goodness of $v$, there exists $g_{1}$ in $[0, v]$ such that $\tau\left(g_{0}\right)=\tau\left(g_{1}\right)$. Set $g=g_{0}-g_{1}$, so that $g \in \operatorname{ker} \tau$, and $\|\widehat{g}-h\| \leq\left\|\widehat{g}_{0}-h\right\|+\left\|\widehat{g}_{1}\right\| \leq \epsilon+\|\widehat{v}\|$.

(ii) By approximate divisibility, there exists $g_{1}$ in $G$ such that $\left\|\widehat{g}_{1}-h\right\|<\epsilon / 3$. Without loss of generality, $\epsilon<\tau(v)$, and thus $\left|\tau\left(g_{1}\right)\right|<\epsilon / 3<\tau(v)$. There exists an order unit $w$ in $G^{+}$such that $\epsilon / 3<\widehat{w}<2 \epsilon / 3$, so that with $g_{0}=g_{1}+w$, we have $0 \leq \tau\left(g_{0}\right)<\epsilon$. By goodness of $v$, there exists $g_{2}$ in $[0, v]$ such that $\tau\left(g_{0}\right)=\tau\left(g_{2}\right)$. Set $g=g_{0}-g_{2}$, so that $g \in \operatorname{ker} \tau$, and $\|\widehat{g}-h\| \leq\left\|\widehat{g}_{0}-h \mid+\right\| \widehat{g}_{2}\|\leq \epsilon+\| \widehat{v} \|$.

Corollary 1.11 (Order unit goodness criterion). Suppose $(G, u)$ is an approximately divisible dimension group with trace $\tau$. Either of the following conditions is sufficient for $\tau$ to be order unit good:

(i) $\tau$ is pure and there exists a sequence of weakly $\tau$-good positive elements of $G,\left\{v_{i}\right\}$, such that $\left\|\widehat{v}_{i}\right\| \rightarrow 0$ and $\tau\left(v_{i}\right)>0$ for almost all $i$.

(ii) There exists a sequence of $\tau$-good positive elements of $G,\left\{v_{i}\right\}$, such that $\left\|\widehat{v}_{i}\right\| \rightarrow 0$ and $\tau\left(v_{i}\right)>0$ for almost all $i$.

Both conditions are of course necessary. This suggests a measurement of how far away a pure trace is from being order unit good; if $u$ is an order unit, use the norm obtained from the unital affine map $G \rightarrow \operatorname{Aff} S(G, u)$, and define the element of $[0, \infty]$ via

$$
\mathcal{G}(\tau) \equiv \mathcal{G}_{u}(\tau)=\inf \left\{\|v\| \mid v \in G^{+} \backslash(\operatorname{ker} \tau) \text { and } v \text { is good with respect to } \tau\right\} .
$$

This depends on the choice of $u$. Then $\tau$ is order unit good if and only if $\mathcal{G}(\tau)=0$, and no positive element outside $\operatorname{ker} \tau$ is weakly $\tau$-good if and only if $\mathcal{G}(\tau)=\infty$. The latter applies when $\tau$ is bad. As a function on $\partial_{e} S(G, u)$ (the extremal boundary of the trace space), $\mathcal{G}$ is mysterious (unless $G$ is a rational vector space, in which 
case $\mathcal{G}$ is $\{0, \infty\}$-valued). If $\tau$ is pure, we can define a similar function replacing good by weakly good.

Corollary 1.12. Let $(G, u)$ be a dimension group and $\tau$ a pure trace thereon. If there exists a weakly $\tau$-good $v$ in $G^{+}$such that $\tau(v) \neq 0$, then the trace on $G \otimes \mathbf{Q}$ (the divisible hull of $G$ ) given by $\tau \otimes 1$ is order unit good.

Proof. Since $G \otimes \mathbf{Q}$ is approximately divisible, it suffices to show each $v_{n}:=v \otimes 1 / n$ is weakly $\tau$-good. However, it is routine to show that the interval $\left[0, v_{n}\right]$ in $G \otimes \mathbf{Q}$ is just $1 / n \cdot\left[0, v_{1}\right]$, and $\left[0, v_{1}\right]=[0, v] \otimes([0,1] \cap \mathbf{Q})$.

Here, $\mathbf{Q}$ can be replaced by any noncyclic subgroup of $\mathbf{Q}$. More criteria for goodness will be obtained in the next section. The converse of Corollary 1.12, whether $\tau \otimes 1$ being good as a pure trace of $G \otimes \mathbf{Q}$ implies there exists a faithful weakly $\tau$-good element in $G$, seems unlikely, but as yet we have found no counterexamples.

\section{A Fistful of tRACES}

With the last few results in mind, we say a trace $\tau$ on a dimension group is ugly if the dimension group has more than one trace, the range of $\operatorname{ker} \tau$ in $\{\tau\}^{\vdash}$ is discrete, and the trace $\tau \otimes 1$ on $G \otimes \mathbf{Q}$ is good. In the case that $G$ is simple, the last condition is equivalent to the divisible hull of $\operatorname{ker} \tau$ (that is, its tensor product with the rationals) having dense range in $\{\tau\}^{\vdash}$. By Law84, a countable abelian group with a discrete norm is free; this applies to the image of $\operatorname{ker} \tau$ when the simple dimension group is countable (as here) and the pure trace $\tau$ is ugly. Thus $\operatorname{ker} \tau$ splits as an abelian group direct sum of a free group and $\operatorname{Inf}(G)$.

A dimension group is critical (see Han82b for properties and examples) if it is simple, has $d$ pure traces, and is free of rank $d+1$. If $G$ is critical, then any subgroup of lesser rank has discrete image in $\mathbf{R}^{d}$. Hence none of its pure traces are good.

For example, if $G$ is a critical group of the form $G=\left\langle e_{1}, \ldots, e_{n} ; \sum_{j} \alpha_{j} e_{j}\right\rangle$ where $e_{i}$ are the standard basis elements in $\mathbf{R}^{n}$ and $\left\{1, \alpha_{1}, \ldots, \alpha_{n}\right\}$ is linearly independent over the rationals, then all pure traces (the coordinate maps) are ugly. It is easy to verify that the element $u=\sum e_{i}=(1,1, \ldots, 1)$ is good with respect to every pure trace. More generally, if $v=\left(v_{i}\right) \in G$ with $v_{i} \geq 1$ for all $i$, then $v$ is good with respect to every pure trace, and thus the function $\mathcal{G}_{u}: \partial_{e} S(G, u)=\{1,2, \ldots, n\} \rightarrow$ $[0, \infty]$ defined earlier is everywhere at most one, and is probably the constant 1 . In this case, we have positive elements that are uniformly good, that is, simultaneously good with respect to every pure trace.

Other types of critical groups of rank $n+1$ can admit no or only some ugly traces.

Recall that a trace is bad if $\operatorname{ker} \tau=\operatorname{Inf}(G)$ and the dimension group has more than one trace. This is preserved by tensoring with the rationals, so bad traces remain bad (not only is the trace not good, it's ba-ad, Proposition 1.5). Bad traces are related to the notion of saturated minimal homeomorphisms, introduced in BK00, which refer to the set of all invariant measures, not just a single one.

Here is a different kind of critical group with all of its pure traces bad. Let $f$ be an irreducible monic polynomial over $\mathbf{Q}$ in one variable with $d+1$ distinct real roots, $\left\{r_{i}\right\}_{i=1}^{d+1}$. Discard the last one and form the elements $v_{j}=\left(r_{1}^{j}, r_{2}^{j}, r_{3}^{j}, \ldots, r_{d}^{j}\right)$ of $\mathbf{R}^{d}$ 
for $j=0,1, \ldots, d$, and set $G=\left\langle v_{j}\right\rangle$. A simple Vandermonde determinant argument (executed in [EHCLS80]) shows that $G$ is a dense subgroup of $\mathbf{R}^{d}$, so that equipped with the strict ordering, $G$ is a simple dimension group and a critical group. The pure traces are the coordinate functions, and since for each $i,\left\{r_{i}^{0}, r_{i}^{1}, \ldots, r_{i}^{d}\right\}$ is linearly independent over $\mathbf{Q}$, it follows that each pure trace has zero kernel, that is, each pure trace is bad.

We now construct two examples, each with exactly three pure traces, such that one is good, one is ugly, and one is bad. Both are free abelian groups, the first of rank five (smallest possible for this arrangement); the second is of infinite rank, because it has an additional property: the value groups of the pure traces, $\tau(G)$, are identical. If $G$ has a bad trace and a not bad trace whose images in $\mathbf{R}$ are equal, then $G$ is isomorphic (as a group) to a proper quotient of itself and, being torsion-free, cannot be of finite rank.

Example 3. (a) A simple dimension group free of rank five with exactly three pure traces: one is good, one is ugly, and one is bad.

(b) A simple dimension group $G$ with exactly three pure traces, $\tau_{i}$, such that one is good, one is ugly, and one is bad, and the value groups $\tau_{i}(G)$ are equal to each other.

Proof. (a) Let $\{a, b, c, d, e, f, g\}$ be an algebraically independent set of seven real numbers. Define the following five elements of $\mathbf{R}^{3}$ :

$$
\begin{aligned}
& v_{1}=\left(\begin{array}{lll}
0 & b & g
\end{array}\right), \\
& v_{2}=\left(\begin{array}{lll}
0 & 1 & f
\end{array}\right), \\
& v_{3}=\left(\begin{array}{lll}
0 & d & e
\end{array}\right), \\
& v_{4}=\left(\begin{array}{lll}
a & b & c
\end{array}\right), \\
& v_{5}=\left(\begin{array}{lll}
1 & 1 & 1
\end{array}\right) .
\end{aligned}
$$

Note that 1 and $b$ are repeated in the second column. Let $G$ be the subgroup of $\mathbf{R}^{3}$ generated by $\left\{v_{i}\right\}$. We first claim that $\left\langle v_{5}, v_{4}, v_{3}, v_{2}\right\rangle$ is a critical subgroup, i.e., is dense. To check this, we note that the set of four $3 \times 3$ determinants is linearly independent over $\mathbf{Q}$. Thus $G$ itself is dense, so with the strict ordering, is a simple dimension group.

Let $\tau_{j}(j=1,2,3)$ be the three coordinate maps. These are the pure traces; we will show that $\tau_{1}$ is good, $\tau_{2}$ is ugly, and $\tau_{3}$ is bad.

Since $\{1, a\}$ is linearly independent over $\mathbf{Q}, \operatorname{ker} \tau_{1}$ is spanned by $\left\{v_{1}, v_{2}, v_{3}\right\}$; we claim ker $\tau_{1}$ has dense image in $\mathbf{R}^{2}$ (after deleting the first coordinate). This corresponds to showing $\langle(b, g),(1, f),(d, e)\rangle$ is dense in $\mathbf{R}^{2}$; the three determinants are $\{b f-g, b e-g d, e-d f\}$, which is clearly linearly independent over $\mathbf{Q}$ (as the original set of seven elements is algebraically independent). Hence ker $\tau_{1}$ has dense range in $\tau_{1}^{\perp}$, so $\tau_{1}$ is good.

As $\{1, b, d\}$ is linearly independent over $\mathbf{Q}$, $\operatorname{ker} \tau_{2}=\left\langle v_{1}-v_{4}, v_{2}-v_{5}\right\rangle$. After deleting the middle zeros, we must show the subgroup $\langle(-a, g-c),(-1, f-1)\rangle$ is discrete and spans $\mathbf{R}^{2}$, i.e., the set $\{(-a, g-c),(-1, f-1)\}$ is a basis of $\mathbf{R}^{2}$. It is linearly independent (as the determinant is $a(1-f)+g-c$, obviously not zero), thus a basis. Hence $\tau_{2}$ is ugly.

Finally, $\operatorname{ker} \tau_{3}$ is zero, since $\{1, c, e, f, g\}$ is linearly independent over the rationals. So $\tau_{3}$ is bad.

Since critical groups cannot have a good trace, this example is the smallest possible (at least in terms of rank) among free abelian simple dimension groups. 
(b) Let $G$ be the free abelian group on the countably infinite set $\left\{x_{i}\right\}_{i \in \mathbf{N}}$. Let a bijection $\mathbf{N} \rightarrow \mathbf{N} \times\{1,2\}$ be denoted $i \mapsto(s(i), l(i))$, and indicate a bijection $\mathbf{N} \times\{1\} \rightarrow \mathbf{N} \times\{3,4\}$ by $(i, 1) \mapsto(t(i), m(i))$. Let $\left\{\alpha_{i}\right\}_{i \in \mathbf{N}}$ and $\left\{\beta_{i}\right\}_{i \in \mathbf{N}}$ be infinite subsets of $\mathbf{R}$ whose union is algebraically independent over $\mathbf{Q}$ (this is probably overkill; it is likely we could get by with linear independence over $\mathbf{Q}$ ).

Now we define three group homomorphisms $\tau_{j}: G \rightarrow \mathbf{R}$, which will turn out to be the desired pure traces. They are of course uniquely determined by their values on $\left\{x_{i}\right\}$ :

$$
\begin{aligned}
& \tau_{1}\left(x_{i}\right)= \begin{cases}0 & \text { if } l(i)=2, \\
\alpha_{t(i)} & \text { if } l(i)=1 \text { and } m(i)=3, \\
\beta_{t(i)} & \text { if } l(i)=1 \text { and } m(i)=4,\end{cases} \\
& \tau_{2}\left(x_{i}\right)= \begin{cases}0 & \text { if } i=\min \{j \mid l(j)=1\}, \\
0 & \text { if } i=\min \{j \mid l(j)=2\}, \\
\alpha_{s\left(i^{\prime}\right)} & \text { where } i^{\prime} \text { is the immediate predecessor to } i \text { in } l^{-1}(1), \\
\beta_{s\left(i^{\prime}\right)} & \text { where } i^{\prime} \text { is the immediate predecessor to } i \text { in } l^{-1}(2),\end{cases} \\
& \tau_{3}\left(x_{i}\right)= \begin{cases}\alpha_{s(i)} & \text { if } l(i)=1, \\
\beta_{s(i)} & \text { if } l(i)=2 .\end{cases}
\end{aligned}
$$

We first observe that $\tau_{j}(G)=\sum \alpha_{i} \mathbf{Z}+\sum \beta_{i} \mathbf{Z}$, so all three group homomorphisms have the same value group. Now consider the map $T:=\left(\tau_{1}, \tau_{2}, \tau_{3}\right): G \rightarrow \mathbf{R}^{3}$. Then $\operatorname{ker} \tau_{3}=0$ is immediate from the construction (this requires only linear independence over $\mathbf{Q}$ ), and we will show that $\operatorname{ker} \tau_{2}$ has discrete range of rank two in $\mathbf{R} \times\{0\} \times \mathbf{R}$ and ker $\tau_{1}$ has dense image in $\{0\} \times \mathbf{R}^{2}$. Finally, we show that the image of $G$ is dense in $\mathbf{R}^{3}$, so that equipped with the strict ordering from the map $T, G$ becomes a simple dimension group. It is then routine that the pure traces are exactly the $\left\{\tau_{j}\right\}$.

Let $i_{1}=\min l^{-1}(1)$ and $i_{2}=\min l^{-1}(2)$; note that $i_{1} \neq i_{2}$ because the relevant map is a bijection. Then linear independence of $\left\{\alpha_{i}, \beta_{j}\right\}$ yields $\operatorname{ker} \tau_{2}=\left\langle x_{i_{1}}, x_{i_{2}}\right\rangle$. The respective images under $T$ are $\left(\gamma, 0, \alpha_{s\left(i_{1}\right)}\right)$ and $\left(0,0, \beta_{s\left(i_{2}\right)}\right)$, where $\gamma$ is one of $\left\{\alpha_{t\left(i_{1}\right)}, \beta_{t\left(i_{1}\right)}\right\}$-all that matters at the moment is that $\gamma \neq 0$. Then $\left\{T\left(x_{1}\right), T\left(x_{2}\right)\right\}$ is linearly independent over $\mathbf{R}$, so that it generates a discrete subgroup of $\mathbf{R}^{3}$. On the other hand, the subgroup it generates sits inside $\mathbf{R} \times\{0\} \times \mathbf{R}$, hence its real span is all of this, and thus its rational span is dense in it.

Now ker $\tau_{1}$ contains $\left\langle x_{j} \mid l(j)=2\right\rangle$ (in fact, the latter is all of ker $\tau_{1}$, but we do not need this). If $j \neq i_{2}$, then for $j \in l^{-1}(2), T\left(x_{j}\right)=\left(0, \beta_{s\left(j^{\prime}\right)}, \beta_{s(j)}\right)$, and $T\left(x_{i_{2}}\right)=\left(0,0, \beta_{s\left(i_{2}\right)}\right)$. Computing a few size two determinants, we see easily that the span of $\operatorname{ker} \tau$ is dense in $\{0\} \times \mathbf{R}^{2}$ (this is where we use algebraic independence, rather than just linear independence, over the rationals, but we could get around this by specifying some of the values).

From the preceding, the closure of $T(G)$ contains $\{0\} \times \mathbf{R}^{2}$, and it also has lots of elements whose first coordinates generate a dense subgroup of $\mathbf{R}$. Since the closure is a group, it follows that $T(G)$ is dense in $\mathbf{R}^{3}$.

Thus we can define the ordering on $G$ via $G^{+} \backslash\{0\}=T^{-1}\left(\left(\mathbf{R}^{3}\right)^{++}\right)$; that is, a nonzero element of $G$ is in the positive cone if and only if its image under $T$ is a strictly positive triple. Density of $T(G)$ ensures that $G$ is a simple dimension group, and it is clear that $\tau_{i}$ are all traces. Moreover, since the ordering is determined by 
the $\tau_{i}$ and they form a linearly independent set, it is trivial that they are pure, and the only pure traces of $G$.

Now knowing that $\tau_{i}$ are pure traces, what we showed above is that they are respectively bad $\left(\tau_{3}\right)$, ugly $\left(\tau_{2}\right)$, and good $\left(\tau_{1}\right)$.

A criterion which can be useful (see the argument for tensor products, Proposition 5.2) is the following.

Lemma 2.1. Let $G$ be an approximately divisible dimension group and $\tau$ a pure normalized trace with respect to a fixed order unit. Suppose in the corresponding affine representation, we have:

Given $\epsilon^{\prime}>0$, there exists $0<\epsilon \leq \epsilon^{\prime}$ for which, given $r \in \tau\left(G^{+}\right) \cap$ $(0, \epsilon)$, there exists $g$ in $G$ such that $\tau(g)=r$ and $\|\widehat{g}\|<f(\epsilon)$ where $\lim _{t \downarrow 0} f(t)=0$.

Then the image of $\operatorname{ker} \tau$ is dense in $\{\tau\}^{\perp}$, and thus $\tau$ is order unit good.

Proof. It suffices to approximate nonnegative elements of $\{\tau\}^{\perp}$ by images of elements of $\operatorname{ker} \tau$, since $\{\tau\}^{\perp}$ is an order ideal, and is thus the abelian group generated by its positive cone. Since $\widehat{G}^{+}$is dense in the set of nonnegative affine functions, for $h$ in $\left(\{\tau\}^{\perp}\right)^{+}$, given one of the $\epsilon^{\prime}$ s that arise from some $\epsilon^{\prime}$, there exists $g_{1}$ in $G^{+}$such that $\left\|\widehat{g}_{1}-h\right\|<\epsilon$. Then $\tau\left(g_{1}\right)=\left(\widehat{g}_{1}-h\right)(\tau)<\epsilon$. Hence we may find $g_{2}$ in $G$ such that $\tau\left(g_{2}\right)=r$ and $\left\|\widehat{g}_{2}\right\|<f(\epsilon)$. Then $w:=g_{1}-g_{2}$ is in $\operatorname{ker} \tau$, and $\|h-\widehat{w}\| \leq\left\|h-\widehat{g}_{1}\right\|+\left\|\widehat{g}_{2}\right\|<\epsilon+f(\epsilon)$.

The $g$ in the displayed hypothesis need not be positive, and moreover, if $f(\epsilon)$ is chosen to be something like $10 \epsilon$, the hypothesis is a weak form of goodness. Here is a slightly different version of the same idea, almost tautological.

Proposition 2.2 (Density criterion). Suppose $(G, u)$ is an approximately divisible dimension group with order unit, and $\tau$ is a pure trace. Sufficient for density of the image of $\operatorname{ker} \tau$ in $\{\tau\}^{\perp}$ is:

For any sequence $\left\{g_{i}\right\}_{i \in \mathbf{N}}$ of elements of the positive cone such that $\tau\left(g_{i}\right) \rightarrow 0$ and $\left\{\widehat{g}_{i}\right\}$ converges uniformly in Aff $S(G, u)$, there exists an infinite subsequence $S \subseteq \mathbf{N}$ together with elements of $G$, $\left\{g_{s}^{\prime}\right\}_{s \in S}$, such that $\tau\left(g_{s}^{\prime}\right)=\tau\left(g_{s}\right)$ for all $s$ in $S$ and $\widehat{g}_{s}^{\prime} \rightarrow 0$ uniformly.

Remark. In the statement, $\tau$ need not be normalized with respect to $u$. The conditions are independent of the choice of order unit, since the norms induced by different order units are mutually equivalent (but changing the order unit can change the isomorphism class of the Choquet simplex $S(G, u)$ - fortunately, this is not relevant here).

Proof. For $h$ in $\left(\{\tau\}^{\perp}\right)^{+}$, density of $\widehat{G}^{+}$in Aff $S(G, u)$ (a consequence of approximate divisibility) allows us to find a sequence $\left\{g_{i}\right\}$ of elements of $G^{+}$such that $\widehat{g}_{i} \rightarrow h$ uniformly; obviously $\tau\left(g_{i}\right) \rightarrow 0$, so we may apply the condition. Setting $w_{s}=g_{s}-g_{s}^{\prime}$, we see that the $w_{s}$ belong to $\operatorname{ker} \tau$ and $\left\|h-\widehat{w}_{s}\right\| \leq\left\|h-\widehat{g}_{s}\right\|+\left\|\widehat{g}_{s}^{\prime}\right\|$, so $\widehat{w}_{s} \rightarrow h$ uniformly. 


\section{A FEW TRACES MORE}

Recall that a Choquet simplex $K$ is a Bauer simplex if its set of extreme points, $\partial_{e} K$, is closed (hence compact).

Proposition 3.1. Suppose that $A$ is a partially ordered ring with 1 as order unit that is also an approximately divisible dimension group. Then:

(i) a normalized trace of $(A, 1)$ is pure if and only if it is multiplicative;

(ii) $S(A, 1)$ is a Bauer simplex and Aff $S(A, 1)$ can be identified with $C(X, \mathbf{R})$ (with the sup norm), where $X=\partial_{e} S(A, 1)$ consists of the pure traces equipped with the point-open (weak) topology;

(iii) a pure trace $\tau$ is order unit good if and only if the ideal of $A, \operatorname{ker} \tau$, is not killed by any pure trace other than $\tau$.

Proof. The only part of this that is not either well known or trivial is the sufficient condition for goodness in part (iii); that is, if $\operatorname{ker} \tau$ does not vanish at any point of $X$ other than $\tau$, then the image of $\operatorname{ker} \tau$ is dense in $\operatorname{Ann}(\tau)=$ $\{f \in C(X, \mathbf{R}) \mid f(\tau)=0\}$. By (i), $\operatorname{ker} \tau$ is an ideal of $A$, therefore its closure in $C(X, \mathbf{R})$ is an ideal thereof. A closed ideal in this algebra is necessarily of the form Ann $(Y)$ for some closed subset $Y$; but the hypothesis forces $Y=\{\tau\}$, so $\operatorname{ker} \tau$ is dense in $\operatorname{Ann}(\tau)$.

Examples (that we will discuss in more detail later) are rational polynomial rings $A=\mathbf{Q}[x]$ or $\mathbf{Q}[x, y]$ which are embedded in $C(I, \mathbf{R}), C(I \times J, \mathbf{R})$ respectively, where $I$ and $J$ are compact subsets of the reals. The images are dense, so by imposing the strict ordering, $A$ becomes a simple dimension group, and it is easy to check that in all these cases, $A$ is a partially ordered ring in which 1 is an order unit, and moreover, $\partial_{e} S(A, 1)$ can be identified with respectively $I$ and $I \times J$.

Example 4. Partially ordered fields with disconnected pure trace space. All pure traces are bad, except when there is just one.

This class of examples is from Han13. Let $K$ be a subfield of the reals, equipped with the sum of squares ordering (that is, an element is in the positive cone if and only if it can be expressed as a sum of squares of elements of $K$ ). If $K$ is algebraic over $\mathbf{Q}$, then $K$ is a simple dimension group with 1 as an order unit, and the pure traces given by the real embeddings of $K$. Thus the pure traces, being multiplicative, must have zero kernel, so all pure traces are bad. In particular, if $K$ is the field of all real numbers algebraic over $\mathbf{Q}$, the pure trace space is an atomless totally disconnected compact set (a Cantor set).

This is an example of an extremely simple dimension group (a simple dimension group for which all pure traces have zero kernel). It is known that the pure trace spaces of these can contain no connected subset; however, this is of less significance than would be expected, since the trace space need not be a Bauer simplex. This class is contained in the class of archimedean simple dimension groups (equivalent to: $\tau(g) \geq 0$ for all pure traces $\tau$ implies $g \in G^{+}$), discussed more fully in Han13. For these, the trace space can be any metrizable Choquet simplex.

Amusing remarks. Suppose $G$ is a simple dimension group with two pure traces, $\tau$ and $\sigma$; for goodness, we may assume that $\operatorname{Inf}(G)=\{0\}$.

(0) General case with two pure traces. The pure trace $\tau$ is good if and only if $\operatorname{ker} \tau$ is not cyclic (that is, $\tau$ is good if and only if $\operatorname{ker} \tau$ is either of rank more than one, 
or $\operatorname{ker} \tau$ is rank one and not finitely generated): since $\operatorname{ker} \tau \cap \operatorname{ker} \sigma=\operatorname{Inf}(G)=\{0\}$, $\sigma$ is one to one on $\operatorname{ker} \tau$, and will have dense range (as a subgroup of the reals) if and only if $\operatorname{ker} \tau$ is not cyclic. Since $\{\tau\}^{\perp}$ is just a copy of the reals (the closure of $\sigma(G)$, as ${ }^{\wedge}$ is given by $\left.(\sigma, \tau)\right)$, we are done. It follows that for simple dimension groups with exactly two pure traces, every trace is one of good, ugly, or bad.

(a) $G$ is not free, but $\operatorname{rank} G=2$. If $\operatorname{ker} \phi=0, \phi$ is bad and thus obviously not good. However, if $\operatorname{ker} \tau \neq 0$, then $G$ is a group extension, $0 \rightarrow L=\operatorname{ker} \tau \rightarrow G \rightarrow$ $\tau(G) \rightarrow 0$, and both $L$ and $\tau(G)$ are rank one. Even though $G$ is dense in $\mathbf{R}^{2}$, examples exist for which $L$ is discrete and nonzero, and in this case, the trace is ugly.

Here is a special class of examples. Let $(a(i)),(b(i))$ be two sequences of positive integers, and set $M_{j}=\left(\begin{array}{ll}a(j) & b(j) \\ b(j) & a(j)\end{array}\right)$. Form $G=\lim M_{j}: \mathbf{Z}^{2} \rightarrow \mathbf{Z}^{2}$. Then $G$ is simple, since the coefficients are all strictly positive. If we multiply any subcollection of the individual terms by $P=\left(\begin{array}{ll}0 & 1 \\ 1 & 0\end{array}\right), G$ remains unchanged. Hence we may assume $b(i) \leq a(i)$ for all $i$. Then $G$ has either two pure traces or one pure trace. Set $\alpha_{k}=\prod_{i>k}(1-b(i) /(a(i)+b(i)))$. If $\alpha_{k}=0$ for all $k$ (that is, $\sum b(i) /(a(i)+b(i))=$ $\infty$ ), then $G$ has unique trace; if there exists $k$ such that $\alpha \equiv \alpha_{k} \neq 0$ (that is, $\sum b(i) /(a(i)+b(i))$ converges $)$, then $G$ has two pure traces; these are constructed from the rows of the limiting matrix of $\prod_{j \geq k} M_{j} /(a(j)+b(j))$. In this case, the automorphism of $G$ induced by $P$ simply switches the pure traces. Hence they are either simultaneously good or simultaneously bad.

Now we claim that the two pure traces are good if and only if $\alpha$ is rational; otherwise they are bad. It easily follows from the construction that the rank of the group of values is two if $\alpha$ is irrational, but one if $\alpha$ is rational. Hence $\operatorname{ker} \tau$ is zero if and only if $\alpha$ is irrational, and otherwise it is rank one but not cyclic (this is easy to see). Hence our criterion applies. Thus if $\alpha \neq 0$, almost certainly the traces will be bad (any number in the interval $(0,1)$ can be attained by $\alpha$ within this class of constructions).

Traditional examples (discussed in the literature) occur with $a(j)=2^{j}$ or $a(j)=$ $j^{2}$ and $b(j)=1$.

Now consider the case that $G$ be free of rank $n$ (and still with exactly two pure traces). Then $n \geq 3$. For every trace, $\operatorname{rank} \tau(G) \geq 2$, and of course, $\operatorname{rank} \operatorname{ker} \tau=$ $n-\operatorname{rank} \tau(G)$. Hence $\tau$ will be good if and only if $\operatorname{rank} \tau(G) \leq n-2$. It will be ugly if and only if $\operatorname{rank} \operatorname{ker} \tau=1$, which is easily arranged.

(b) $n=3$. Then for every trace $\phi, \operatorname{rank} \phi(G) \geq 2$, so neither trace is good.

(c) $n=4$. Then $\phi$ is good if and only if $\operatorname{rank} \phi(G)=2$. Thus we can arrange that one of the traces be good and the other not good. Let $\left\{\alpha, \beta_{1}, \beta_{2}\right\}$ be a set of real numbers such that each of the two sets, $\left\{1, \beta_{1}, \beta_{2}\right\}$ and $\left\{\alpha, \beta_{1}, \alpha \beta_{1}\right\}$, is linearly independent over the rationals (this implies $\{1, \alpha\}$ is also linearly independent); let $G$ be the subgroup of $\mathbf{R}^{2}$ generated by $\left\{(1,1),(\alpha, 0),\left(0, \beta_{1}\right),\left(0, \beta_{2}\right)\right\}$. Density in $\mathbf{R}^{2}$ comes from $\left\{\alpha, \beta_{1}, \alpha \beta_{1}\right\}$ being linearly independent; equip $G$ with the strict ordering, making it into a simple dimension group, with the two pure traces, $\sigma=\tau_{1}$ and $\tau=\tau_{2}$, the coordinate projections.

It follows from linear independence of $\left\{1, \beta_{1}, \beta_{2}\right\}$ over $\mathbf{Q}$ that $\operatorname{ker} \tau_{2}$ is spanned by $(\alpha, 0)$; so rank ker $\tau_{2}=1$ and thus $\tau_{2}$ is ugly, therefore not good. On the other hand, $\tau_{1}(G)$ is spanned by $\{1, \alpha\}$, so $\tau_{1}$ is good. We can obviously modify this example so that both traces are good. 
Example 5. Simple dimension groups with a continuum of pure traces, for which the set of good traces, and the set of not good traces, are both dense, and the former is countable.

Let $G=\mathbf{Q}[x]$, and let $I$ be a compact subset of $\mathbf{R}$; the typical (but not the only) examples for $I$ will be intervals, $[a, b]$, in the reals. Put the strict ordering on $G$ resulting from restricting to $I$, that is, a nonzero polynomial $p$ is in the positive cone if and only if $p$ is strictly positive as a function on $I$. Density of $\mathbf{Q}[x]$ in $\mathbf{R}[x]$ (which by Weierstrass' theorem is dense in $C(I, \mathbf{R})$ ) ensures that $G$ is a simple dimension group. The pure traces are given by evaluations at the points of $I$, that is, $\tau_{\alpha}: p \mapsto p(\alpha)$ for $\alpha$ in $I$, and the norm on the affine function space translates to the sup norm on functions on $I$. It is easy to check that $\left\{\tau_{\alpha}\right\}^{\perp}$ is just the ideal consisting of the continuous functions on $I$ that vanish at $\alpha$. Assume $I$ has at least two points.

If $\alpha$ is transcendental, then $\tau_{\alpha}$ has kernel zero, so is bad. If $\alpha$ is rational, then the density criterion of Proposition 2.2 (which after an affine transformation, amounts to showing $x \mathbf{Q}[x]$ is dense in the continuous functions on $[0,1]$ that vanish at zero; this follows from $x \mathbf{R}[x]$ being dense) applies, so that $\tau_{\alpha}$ is good.

If $\alpha$ is algebraic but not rational, the situation is more interesting. Let $f_{\alpha}$ be the monic irreducible (over the rationals) polynomial of $\alpha$. Then $\operatorname{ker} \tau_{\alpha}$ is the ideal $f_{\alpha} \mathbf{Q}[x]$. If $f_{\alpha}$ has another root, say $\beta$, in $I$, then $\tau_{\beta}$ vanishes on $\operatorname{ker} \tau_{\alpha}$ (because the kernels are equal), so $\operatorname{ker} \tau_{\alpha}$ cannot be dense in $\left\{\tau_{\alpha}\right\}^{\perp}$. Thus when $f_{\alpha}$ has another root in $I, \tau_{\alpha}$ is not good (but not bad either).

Finally, suppose that $f_{\alpha}$ has just the one root in $I$; then $\tau_{\alpha}$ is good. To see this, note that it suffices to show $x-\alpha$ belongs to the closure of $f_{\alpha} \mathbf{Q}[x]$ (for then $(x-\alpha) \mathbf{R}[x]$ will also be contained in the closure, and this is dense in $\left.\left\{\tau_{\alpha}\right\}^{\perp}\right)$. Consider the function $f_{\alpha} /(x-\alpha)$; this is continuous on $I$ and has no zeros there by hypothesis. Its reciprocal, $h:=(x-\alpha) / f_{\alpha}$, is thus continuous, hence may be approximated by elements of $\mathbf{Q}[x]$, say $g_{n} \rightarrow h$ uniformly on $I$. Then $\left(g_{n} f_{\alpha}\right)$ is a sequence of elements in $f_{\alpha} \mathbf{Q}[x]$ that converges to $x-\alpha$.

To summarize: $\tau_{\alpha}$ is good if and only if $\alpha$ is algebraic and has no other algebraic conjugates in $I$. For example, if $I=[a, 2]$ for some $a \leq \sqrt{2}$ and $\alpha=\sqrt{2}$, then for our $G$ (which now depends on $a$ ), $\tau_{\alpha}$ is good if and only if $-\sqrt{2}<a \leq \sqrt{2}$ (if $a>\sqrt{2}$, then $\tau_{\sqrt{2}}$ is not a trace). The bad traces are exactly the evaluations at transcendentals in $I$; there are no ugly traces, since the dimension group is a rational vector space.

We can put a finer dimension group ordering on $\mathbf{Q}[x]$; take the pointwise ordering on $[a, b]$, that is, $g \geq 0$ if and only if $g$ is nonnegative on the interval. This is a dimension group (over the reals, this is due to Fuchs; see [H7]), with the same pure traces, but of course it is not simple. In fact, if $\alpha$ is rational, then $\operatorname{ker} \tau_{\alpha}$ is a maximal order ideal. The $\{\tau\}^{\perp}$ are exactly the same, and the same characterization of good traces applies.

If the interval $I=[a, b]$ contains no integers, then we can do the same with $G=\mathbf{Z}[x]$ (the point being that $\mathbf{Z}[x]$ is norm dense in $\mathbf{R}[x]$ in this case).

In contrast, there is an explicit (and also an inexplicit) construction Han13 of an archimedean simple dimension group (necessarily not an ordered ring) whose pure trace space is $[0,1]$, such that all evaluations at algebraic points have zero kernel 
(and therefore are bad). Point evaluations at transcendentals may have kernels, but it is not clear whether any of them are good.

It is a plausible conjecture that if $\tau$ is a pure trace on a simple dimension group $G$ with $\operatorname{rank} \tau(G)=1$, then $\tau$ is good. This is true if there exists a subgroup $H$ together with a rank one noncyclic subgroup $U$ of $\mathbf{Q}$ such that $H \otimes U \subseteq G \subseteq H \otimes \mathbf{Q}$ if $\phi$ is a continuous linear functional on $\operatorname{Aff}(S(G, u))$, then either $\phi(\operatorname{ker} \tau)=0$ or $\phi(\operatorname{ker} \tau)$ contains $U \alpha$ for some nonzero $\alpha$; the latter entails $\phi(\operatorname{ker} \tau)$ is dense and the former that $\phi \mid G$ is a scalar multiple of $\tau$ (since $\phi$ induces a homomorphism to the reals on the rank one group $\tau(G)$; any two nonzero such must be scalar multiples of each other), which is excluded. Hence the image of $\operatorname{ker} \tau$ is dense in $\{\tau\}^{\perp}$.

It is also true if the map $\tau: G \rightarrow U \subseteq \mathbf{Q} \alpha$ (where $U$ is rank one, but not cyclic) is nearly split; that is, if there is a subgroup $J$ of $G$ such that $(\operatorname{ker} \tau) \cap J=\{0\}$ and $G /(J+\operatorname{ker} \tau)$ is finite. Finite index means the problem reduces to $G_{0}=J \oplus \operatorname{ker} \tau$, and since $\tau$ is essentially the projection onto $J$ followed by multiplication by a real number, it easily follows (from the splitting) that $\widehat{G}$ dense in the affine function space implies $\operatorname{ker} \tau$ has dense range in $\{\tau\}^{\perp}$.

However, the conjecture is not true in general. The following example illustrates a few interesting points.

Example 6. A simple dimension group with two pure traces, each ugly and having values in $\mathbf{Z}\left[\frac{1}{2}\right]$; moreover, the underlying abelian group is strongly indecomposable of rank two.

Let $G_{0}$ be the free abelian group on a countably infinite set, $\left\{x_{i}\right\}_{i=0}^{\infty}$. Let $k$ : $\mathbf{Z}^{+} \rightarrow\{0,1\}$ be a function such that the collection of initial segments of all $0-1$ strings of the form $k(i) k(i-1) \ldots k(0) 1$ includes all finite sequences infinitely often, and suppose $k(0)=0$. Define the rational numbers $\alpha_{0}=1$ and $\alpha_{i+1}=\left(\alpha_{i}+k(i)\right) / 2$, and now define two real-valued functions $\tau_{1}, \tau_{2}$ from $G_{0}$ via

$$
\tau_{1}\left(x_{i}\right)=\frac{1}{2^{i}}, \quad \tau_{2}\left(x_{i}\right)=\alpha_{i} .
$$

(i) The range of $T=\left(\tau_{1}, \tau_{2}\right): G_{0} \rightarrow \mathbf{R}^{2}$ is dense. Let $J$ denote the closure of the image of $G_{0}$. We observe that $T\left(x_{i}\right)=\left(2^{-i}, \alpha_{i}\right)$. For any real number $\beta$ in the interval $[0,1]$, there exists an infinite subset $S_{\beta}$ of $\mathbf{Z}^{+}$such that $\lim _{s \in S_{\beta} \rightarrow \infty} \alpha_{s}$ $=\beta$. Then $(0,1 / 2)$ and $(0,1 / e)$ are limit points of $\left\{T\left(x_{s}\right)_{S_{1 / 2}}\right\}$ and $\left\{T\left(x_{s}\right)_{S_{1 / e}}\right\}$ respectively, so that $\{0\} \times\left(\frac{1}{2} \mathbf{Z}+\frac{1}{e} \mathbf{Z}\right)$ is contained in $J$, whence $\{0\} \times \mathbf{R}$ is in $J$. This means that $\left(2^{-i}, 0\right) \in J$, whence the closure of the subgroup it generates contains $\mathbf{R} \times\{0\}$. Since $J$ is a group, $J=\mathbf{R}^{2}$.

(ii) With the strict ordering from $T, G_{0}$ becomes a simple dimension group, and the only pure traces are $\tau_{1}$ and $\tau_{2}$.

(iii) ker $\tau_{1}=\left\langle\left\{2 x_{i}-x_{i-1}\right\}\right\rangle$. Let $g=\sum_{j=0}^{k} m_{j} x_{j} \rightarrow 0$ under $\tau_{1}$ with $m_{k} \neq 0$. Write $m_{k} x_{k}=\left\lfloor m_{k} / 2\right\rfloor\left(2 x_{k}-x_{k-1}\right)+\epsilon(k) x_{k}+\left\lfloor m_{k} / 2\right\rfloor x_{k-1}$, where $\epsilon(k) \in\{0,1\}$. This reduces $g$ to $\sum \epsilon(i) x_{i}$. Since this maps under $\tau$ both to zero and to the binary number $\sum \epsilon(i) 2^{-i}$, it follows that all the $\epsilon(i)=0$, so that $g$ is in the group $\left\langle\left\{2 x_{i}-x_{i-1}\right\}\right\rangle$.

(iv) The range of $\tau_{2}$ on $\operatorname{ker} \tau_{1}$ is cyclic. Since $2 \alpha_{i}-\alpha_{i-1} \in \mathbf{Z}$, we thus have $\tau_{2}\left(2 x_{i}-x_{i-1}\right) \in \mathbf{Z}$, so $\tau_{2}\left(\operatorname{ker} \tau_{2}\right) \subseteq \mathbf{Z}$.

(v) Finally, $\tau_{1}\left(G_{0}\right)=\mathbf{Z}\left[1 / 2^{i}\right] \subseteq \mathbf{Q}$, and since $\tau_{2}\left(\operatorname{ker} \tau_{1}\right)$ is cyclic, $\tau_{1}$ is in fact ugly, not good. 
This example can be modified to a somewhat more startling one. We check that $\operatorname{ker} \tau_{2}=\left\langle\left\{2 x_{i+1}-x_{i}\right\}_{k^{-1}(0)} \cup\left\{2 x_{j+1}-x_{j}-x_{0}\right\}_{k^{-1}(1)}\right\rangle$, so that the image of $\operatorname{ker} \tau_{2}$ is also discrete. Now $\operatorname{ker} \tau_{1} \cap \operatorname{ker} \tau_{2}=\operatorname{Inf}\left(G_{0}\right)$ (since $\tau_{1}$ and $\tau_{2}$ are the only pure traces on $G_{0}$ ), and it is easy to check that $\operatorname{ker} \tau_{1} \cap \operatorname{ker} \tau_{2}$ is spanned by $\left\{2 x_{i+1}-x_{i}\right\}_{k^{-1}(0)} \cup\left\{2 x_{j+1}-x_{j}-\left(2 x_{l+1}-x_{l}\right)\right\}_{j, l \in k^{-1}(1)}$. Let $G$ be $G_{0} / \operatorname{Inf}\left(G_{0}\right)$, and let $\bar{\tau}_{i}$ be the traces corresponding to $\tau_{i}$. Then $\bar{T}:=\left(\bar{\tau}_{1}, \bar{\tau}_{2}\right): G \rightarrow \mathbf{R}^{2}$ is the affine representation mapping, and is a one to one map from $G$ to $\mathbf{Z}[1 / 2] \oplus \mathbf{Z}[1 / 2]$ (with the strict ordering on the latter). In particular, $G$ is rank two, and each of $\operatorname{ker} \bar{\tau}_{i}$ is cyclic (and not zero). Since $T\left(G_{0}\right)=\bar{T}(G)$ is dense in $\mathbf{R}^{2}$, it follows that $G$ is indecomposable, in fact every finite index subgroup is indecomposable as an abelian group.

\section{EXTENSIONS}

This section deals with extensions of traces defined on order ideals of a dimension group $G$ to $G$ itself-whether goodness persists under this process. The following is elementary.

Lemma 4.1. Let $G$ be a dimension group with order unit $u$, and $J$ an order ideal in $G$. Let $\sigma$ be a trace on $J$. Then $\sigma$ extends to a trace on $G$ if and only if

$$
\sup \{\sigma(a) \mid a \in J, \quad 0 \leq a \leq u\}<\infty .
$$

If $\sigma$ is pure and it extends, then it can be extended to a pure trace.

The following generalizes BK11, BKMS10, which applied to stationary systems.

Proposition 4.2. Let $G$ be a dimension group with an order unit, let $J$ be an order ideal of $G$, let $\sigma$ be a pure trace of $J$, and let $\tau$ be a pure trace of $G$ extending $\sigma$. Then $\tau$ is good if and only if

(i) $\sigma$ is good and

(ii) $\sigma\left(J^{+}\right)=\tau\left(G^{+}\right)$.

Proof. Necessity. (i) Select $a, b$ in $J^{+}$with $\sigma(a)<\sigma(b)$. Then $\tau(a)<\tau(b)$, so there exists $0 \leq c \leq b$ in $G$ such that $\tau(a)=\tau(c)$; but $0 \leq c \leq b$ and $b$ belongs to the order ideal $J$, so $c$ belongs to $J$.

(ii) Normalize $\tau$ (and correspondingly $\sigma$ ) so that $\tau(u)=1$. Suppose $r \in \tau\left(G^{+}\right) \backslash$ $\{0\}$; there exists $a$ in $G^{+}$such that $\tau(a)=r$. Since $\sigma$ is not zero, there exists $b_{0}$ in $J^{+}$such that $\sigma\left(b_{0}\right) \neq 0$, hence there exists a positive integer $n$ such that $n \sigma\left(b_{0}\right)>r$; set $b=n b_{0}$ in $J^{+}$. Since $\tau$ is good, there exists $0 \leq c \leq b$ (so $c$ belongs to $J^{+}$) such that $r=\tau(a)=\tau(c)=\sigma(c)$, hence $r \in \sigma\left(J^{+}\right)$.

Sufficiency. Select $a, b$ in $G^{+}$with $\tau(b)>\tau(a)>0$. By (ii), there exists $a^{\prime}, b^{\prime}$ in $J^{+}$such that $\sigma\left(a^{\prime}\right)=\tau(a)$ and $\sigma\left(b^{\prime}\right)=\tau(b)$. By purity of $\tau$, with $\epsilon<\tau(b)-\tau(a)$, there exists $0 \leq b^{\prime \prime} \leq b^{\prime}, b$ such that $\tau\left(b^{\prime \prime}\right)>\tau(b)-\epsilon>\tau(a)$. From $b^{\prime \prime} \leq b^{\prime}$, $b^{\prime \prime}$ belongs to $J$. Applying goodness of $\sigma$ to $b^{\prime \prime}, a^{\prime}$, there exists $0 \leq c \leq b^{\prime \prime}$ with $\sigma(c)=\sigma\left(a^{\prime}\right)$. Thus $c \leq b$ and $\tau(c)=\tau(a)$.

Suppose $\tau$ is a pure trace of $G$. If there exists a minimal order ideal $I$ such that $\tau(I) \neq 0$, then $\tau$ is good if and only if $\tau \mid I$ is good and $\tau\left(I^{+}\right)=\tau\left(G^{+}\right)$. As $I$ is a minimal order ideal and the notion of order ideal is transitive, $I$ is simple as a dimension group. So we have necessary and sufficient conditions for $\tau$ to be good 
(assuming $I$ is minimal and $\tau(I) \neq 0$ ), namely, $\tau\left(I^{+}\right)=\tau\left(G^{+}\right)$and either $I=\mathbf{Z}$, or ker $\tau \mid I$ has dense image in $\{\tau \mid I\}^{\perp}$ (with respect to one or any order unit of $I$ ).

Part of the problem in trying to give a general characterization of good traces for nonsimple dimension groups is that the approximately divisible condition does not behave well on taking order ideals, and the corresponding density even less well. However, we note that the proof of Proposition 1.7 shows that if $b$ is an order unit and the density condition holds, then $b$ is $\tau$-good. This has a consequence: if $\tau$ is a pure trace, and for every order ideal with (relative) order unit $(I, b)$ (that is, $I$ is the order ideal generated by $b$ ), $\operatorname{ker} \tau \mid I$ has dense image in $\{\tau \mid I\}^{\perp}$ (the order ideal of Aff $S(I, b)$ consisting of the functions vanishing at $\tau \mid I)$, and $\tau\left(I^{+}\right)=\tau\left(G^{+}\right)$, then $\tau$ is good.

In this result, we need only do this for one choice of order unit $b$ for each $I$. Moreover, we can weaken this further by using the generating result. Namely, let $H \subseteq G^{+}$be a generating set. Look at all the order ideals with order unit of $G$; we can restrict our interest to the order ideals generated by each element of $H$.

In general, an order ideal $I$ (with order unit) in a dimension group $G$ need not be approximately divisible when $G$ is. One trivial condition that forces order ideals (with order unit) to be approximatively divisible is that $G$ be divisible (for then the order ideals are themselves divisible), or 2-divisible, or have similar conditions (e.g., $G \cong G_{0} \otimes U$, where $U$ is a noncyclic subgroup of the rationals). When every (or sufficiently many) order ideal is approximately divisible, goodness of the restriction of a trace forces the density property. In this case, we would obtain necessary and sufficient conditions for $\tau$ to be a good trace.

For $h$ a positive element of $G$, let $I(h)$ denote the order ideal of $G$ generated by $h$; that is,

$$
I(h)=\{g \in G \mid \text { there exists } K \in \mathbf{N} \text { such that }-K h \leq g \leq K h\} .
$$

Proposition 4.3. Let $G$ be a dimension group, and let $\tau$ be a pure trace. Let $H$ be a generating subset of $G^{+}$.

(a) If for all $h$ in $H$ such that $\tau(h) \neq 0$, (i) $\tau\left(I(h)^{+}\right)=\tau\left(G^{+}\right)$and (ii) the image of $\operatorname{ker} \tau \mid I(h)$ is dense in the order ideal $\{\tau \mid I(h)\}^{\perp}$ of Aff $S(I(h), h)$, then $\tau$ is good.

(b) Suppose that all order ideals of $G$ with order units are approximatively divisible and $\tau$ is good. Then for all order ideals $(I, b)$ with order unit such that $\tau \mid I \neq 0$, it follows that (i) $\tau\left(I^{+}\right)=\tau\left(G^{+}\right)$and (ii) the image of $\operatorname{ker} \tau \mid I$ is dense in the order ideal $\{\tau \mid I\}^{\perp}$ of Aff $S(I, b)$.

These conditions are still unwieldy in general, but work perfectly well if (for example) $G$ has only finitely many order ideals, or if there exists a unique minimal order ideal not killed by $\tau$.

Let $\tau$ be a trace on the dimension group $G$. Define $\operatorname{ker}^{+} \tau$ to be abelian group generated by $\operatorname{ker} \tau \cap G^{+}$(that is, the set of differences of such elements). It is an order ideal of $G$, and is the largest order ideal contained in $\operatorname{ker} \tau$. We may thus form $\bar{G}=G / \operatorname{ker}^{+} \tau$, the dimension group obtained as the quotient by an order ideal; then $\tau$ induces a trace on $\bar{G}$ via $\bar{\tau}\left(g+\operatorname{ker}^{+} \tau\right)=\tau(g)$. It is routine that $\tau$ is pure (as a trace of $G$ ) if and only if $\bar{\tau}$ is pure (as a trace of $\bar{G}$ ). It is equally routine (in fact the argument is almost identical and obvious) that the same result holds for goodness. 
Lemma 4.4. Suppose $G$ is a dimension group, $\tau$ is a trace thereon, and $I$ is an order ideal of $G$ such that $I \subseteq \operatorname{ker} \tau$. If $H=G / I$ with the quotient ordering, and $\tilde{\tau}$ denotes the trace induced on $H$ by $\tau$, then $\tau$ is good (as a trace of $G$ ) if and only if $\tilde{\tau}$ is good (as a trace of $H$ ).

Proof. $(\Rightarrow)$ Given $A, B$ in $H^{+}$such that $\tilde{\tau}(A)<\tilde{\tau}(B)$, there exist $a$ and $b$ in $G^{+}$ whose images in $H$ are respectively $A$ and $B$. Apply goodness to $b$ and $a$, obtaining $0 \leq c \leq b$ such that $\tau(c)=\tau(a)=\tilde{\tau}(A)$; then $C:=c+I$ is the desired element in $H$.

$(\Leftarrow)$ Suppose $a$ and $b$ are in $G^{+}$with $0<\tau(a)<\tau(b)$. Let $A$ and $B$ be their respective images in $H^{+}$. By goodness of $\tilde{\tau}$, there exists $C$ in $H^{+}$such that $C \leq B$ and $\tilde{\tau}(C)=\tilde{\tau}(A)=\tau(a)$. There exists $c_{1}$ in $G^{+}$such that $C=c_{1}+I$. There exists $f$ in $H^{+}$such that $c_{1} \leq b+f$ (from the quotient ordering). By Riesz decomposition, there exist $0 \leq c \leq b$ and $0 \leq f_{1} \leq f$ such that $c_{1}=c+f_{1}$. Obviously, $\tau(c)=\tau\left(c_{1}\right)=\tilde{\tau}(A)=\tau(a)$, so $c$ is the desired element.

Corollary 4.5. If $\tau$ is a trace such that $\operatorname{ker}^{+} \tau=\operatorname{ker} \tau$, then $\tau$ is good. In particular, this applies if $\tau$ is a pure discrete trace.

Proof. If equality holds, then $\bar{G}$ is order isomorphic (via $\bar{\tau}$ ) to a subgroup of the reals, hence is totally ordered and hence has unique trace, which necessarily equals $\bar{\tau}$, and the latter is thus good. The preceding result then applies. For a pure discrete trace, $\operatorname{ker}^{+} \tau=\operatorname{ker} \tau$ Goo86].

More important than the corollary (the condition on $\operatorname{ker} \tau$ seldom holds) is that we can combine this with our previous conditions to check whether $\tau$ is good. Given a pure trace $\tau$ on $G$, factor out $\operatorname{ker}^{+} \tau$, try to verify the previous conditions for the presumably less complicated $\bar{G}$. For example, if the latter has sufficiently many order ideals with order units such that the density condition on the relative kernels of $\bar{\tau}$ applies, and the range of $\bar{\tau}$ on the order ideals equals that on the whole group, then $\bar{\tau}$ (and thus $\tau$ ) is good.

Example 7. (a) A dimension group with a pure trace that is order unit good but not good; (b) an extended homomorphism of dimension groups $G \rightarrow H$ together with a pure trace $\tau$ that is good, but its extension to $H$ is not.

Proof. Let $K$ and $K^{\prime}$ be dense subgroups of the reals, and form an extension (in the category of dimension groups) of $K$ by $K^{\prime}$, following the prescription in Han82a. (developed further in GH82]). Set $G\left(K, K^{\prime}\right)=K \oplus K^{\prime}$ with the following ordering:

$$
\left.G\left(K, K^{\prime}\right)^{+}=\left\{(k, 0) \in K \oplus\{0\} \mid k \in \mathbf{R}^{+}\right\} \cup\left\{(k, l) \in K \oplus K^{\prime}\right) \mid k+l, l \in \mathbf{R}^{++}\right\} .
$$

That this is a dimension group with exactly two pure traces, given by $\tau:(k, l) \mapsto$ $k+l$ and $\sigma:(k, l) \mapsto l$, is folklore, but is discussed in Han82a; moreover, $J:=$ $K \oplus\{0\}$ is the only proper order ideal of $G\left(K, K^{\prime}\right)$, and the quotient by $J$ is $K^{\prime}$. Since $K$ is totally ordered and simple, $\tau \mid J$ is good. It is easy to check directly that $G\left(K, K^{\prime}\right)$ is approximately divisible (this also follows from GH80, which asserts that a dimension group with order unit and no discrete traces is approximately divisible).

Next we look at

$$
\operatorname{ker} \tau=\left\{(k,-k) \mid k \in K \cap K^{\prime}\right\} .
$$

We note that $\{\tau\}^{\perp}=\left\{(r,-r) \in \mathbf{R}^{2}\right\} \subset \mathbf{R}^{2}$. If $K \cap K^{\prime}=\{0\}$, then $\tau$ is bad; if $K \cap K^{\prime}$ is cyclic, then $\operatorname{ker} \tau$ cannot be dense in $\{\tau\}^{\perp}$, so that $\tau$ is not order unit 
good, let alone good. However, if $K \cap K^{\prime}$ is dense in $\mathbf{R}$, then $\sigma(\operatorname{ker} \tau)=K \cap K^{\prime}$ is dense in the reals, so that $\tau$ is order unit good. Thus we have,

(i) $\tau$ is order unit good if and only if $K \cap K^{\prime}$ is dense in $\mathbf{R}$.

Since $\tau(G)=K^{\prime}+K$ but $\tau(J)=K$, we also have from Lemma 4.1 that

(ii) $\tau$ is good if and only if $K^{\prime} \subseteq K$.

Next, we observe that if $K^{\prime} \subseteq K^{\prime \prime}$, then the inclusion $G\left(K, K^{\prime}\right) \subseteq G\left(K, K^{\prime \prime}\right)$ is an extended homomorphism, since the traces extend uniquely.

(a) Suppose that $K^{\prime}$ strictly contains $K$; then $\tau$ is not good by (ii), but is order unit good by (i).

(b) The inclusion $G:=G(K, K) \subset H:=G\left(K, K^{\prime}\right)$ (where $K^{\prime}$ is as in (a)) is extended; however, $\tau$ restricted to $G(K, K)$ is good by (ii), but by (i), its extension to $\tau$ as a pure trace on $G\left(K, K^{\prime}\right)$ is not good.

\section{ORdERED TENSOR PRODUCTS}

If $G$ and $H$ are partially ordered abelian groups, then we may form their ordered tensor product (over $\mathbf{Z}$; this can be done over other ordered rings as well), $G \otimes H$, whose underlying group is that of their tensor product as abelian groups, with positive cone generated by elements of the form $\left\{g \otimes h \mid g \in G^{+}, h \in H^{+}\right\}$. That this $i s$ a partially ordered abelian group is not entirely trivial [GH86], but is trivial if both are dimension groups, and in the latter case is also a dimension group (also trivial to show).

Suppose both $G$ and $H$ have order units, $u$ and $v$ respectively. Then $u \otimes v$ is an order unit for $G \otimes H$, and the trace space is easily determined, although a little awkward. What is not awkward, however, is the pure trace space. This is the cartesian product of the pure trace space, that is, $\partial_{e} S(G \otimes H, u \otimes v)=$ $\partial_{e} S(G, u) \times \partial_{e} S(H, v)$; the identification is via $(\sigma, \tau) \mapsto \sigma \otimes \tau$ (the latter acts via $\sigma \otimes \tau(g \otimes h)=\sigma(g) \tau(h))$. That is, every pure trace of the tensor product is a tensor product of pure traces, and every such tensor product is pure. Now we can ask when $\sigma \otimes \tau$ is good.

If $G$ and $H$ are simple (respectively, approximately divisible), then so is $G \otimes H$. Thus we can apply (some) results above. For example, suppose $G$ and $H$ are simple, and that the two traces $\sigma$ and $\tau$ are both good with respect to their respective groups. We show $\sigma \otimes \tau$ is good.

Lemma 5.1. Suppose $G$ is an approximately divisible dimension group with order unit. If $\tau$ is an order unit good trace, then for all $g$ in $G^{+} \backslash \operatorname{ker} \tau$, there exists a positive element $g^{\prime}$ of $G$ such that $\tau\left(g^{\prime}\right)=\tau(g)$ and $\left\|\widehat{g}^{\prime}\right\| \leq 2 \tau(g)$.

Proof. By the density of $G$ in its affine representation (with respect to a fixed order unit), there exists $b$ in $G$ such that $\tau(g) \mathbf{1}<\widehat{b} \leq 2 \tau(g) \mathbf{1}$ (possible since $\tau(g) \neq 0$ ). Then $\widehat{b}$ being strictly positive implies $b$ is an order unit of $G$, in particular, $b$ belongs to $G^{+}$, and $\tau(b)>\tau(g)$. Apply order unit goodness to $b$ and $g$; there exists $g^{\prime}$ in $G^{+}$such that $\tau\left(g^{\prime}\right)=\tau(g)$ and $g^{\prime} \leq b$. The latter entails $\widehat{g}^{\prime} \leq \widehat{b} \leq 2 \tau(g) \mathbf{1}$, so $\left\|\widehat{g}^{\prime}\right\| \leq 2 \tau(g)$.

Proposition 5.2. Let $G$ and $H$ be approximately divisible dimension groups with order units and with pure traces $\sigma$ and $\tau$ respectively. If $\tau$ and $\sigma$ are order unit good, then the pure trace $\sigma \otimes \tau$ of $G \otimes H$ is order unit good. In particular, if $G$ and $H$ are simple and $\sigma$ and $\tau$ are both good, then $\sigma \otimes \tau$ is good. 
Remark. If merely $G$ is approximately divisible (and both have an order unit), then $G \otimes H$ is approximately divisible. It is not immediately clear how to show that in this case, $\sigma \otimes \tau$ is good if both $\sigma$ and $\tau$ are.

Proof. It is immediate that $G \otimes H$ is approximately divisible. We apply the density criterion to show that $\operatorname{ker} \sigma \otimes \tau$ is dense in $\{\sigma \otimes \tau\}^{\perp}$, and then Proposition 1.7 applies. When $G$ and $H$ are simple, so is $G \otimes H$, and thus order unit good is the same as good.

Fix order units for each of $G$ and $H$, and use the corresponding affine representations; the tensor product of the order units gives an order unit for the tensor product of the groups. Given $g$ in $G^{+}$and $h$ in $H^{+}$, there exist $g^{\prime}, h^{\prime}$ in $G^{+}$and $H^{+}$respectively such that $\sigma\left(g^{\prime}\right)=\sigma(g), \tau\left(h^{\prime}\right)=\tau(h)$, and $\left\|\widehat{g}^{\prime}\right\| \leq 2 \sigma(g)$, and $\|\widehat{h}\| \leq 2 \tau(h)$. Then $g^{\prime} \otimes h^{\prime}$ is positive and $\left\|\widehat{g^{\prime} \otimes h^{\prime}}\right\| \leq 4 \sigma(g) \tau(h)$ with respect to the affine representation for $G \otimes H$; moreover, $\sigma \otimes \tau\left(g^{\prime} \otimes h^{\prime}\right)=\sigma \otimes \tau(g \otimes h)$.

Applying this to all the elements in a sum $z=\sum g_{j} \otimes h_{j}$ (with $g_{j} \in G^{+}$and $h_{j} \in H^{+}$), we can find positive elements $g_{j}^{\prime}$ and $h_{j}^{\prime}$ such that with $z^{\prime}=\sum g_{j}^{\prime} \otimes h_{j}^{\prime}$, we have $\sigma \otimes \tau\left(z^{\prime}\right)=\sigma \otimes \tau(z)$ and $\left\|\widehat{z}^{\prime}\right\|<4 \sigma \otimes \tau(z)$. This is more than enough to verify one of the density criteria (Proposition 2.2) for $\sigma \otimes \tau$ : if $\sigma \otimes \tau\left(z_{i}\right) \rightarrow 0$, then $\left\|\widehat{z}_{i}^{\prime}\right\| \rightarrow 0$.

Here is a rather surprising general result about tensor products of traces.

Proposition 5.3. Let $\sigma, \tau$ be normalized pure traces on the simple dimension groups with order unit, $(G, u),(H, v)$, respectively, and suppose moreover that $\tau$ is good, and $\sigma\left(G^{+}\right) \tau\left(H^{+}\right) \subseteq \tau\left(H^{+}\right)$. Then $\sigma \otimes \tau$ is a good pure trace of $G \otimes H$.

Remark. The condition says that $\tau(H)$ is a unital module over the subring of the reals generated by $\sigma(G)$ (since the traces are normalized, 1 belongs to both $\sigma(G)$ and $\tau(H))$.

Proof. Suppose $z_{i}=\sum_{j} g_{j i} \otimes h_{j i}$ belong to $(G \otimes H)^{+}$(with $g_{j i}$ in $G^{+}$and $h_{j i}$ in $H^{+}$) and $\sigma \otimes \tau\left(z_{i}\right) \rightarrow 0$. Since $\sigma \otimes \tau\left(z_{i}\right)=\sum_{j} \sigma\left(g_{i j}\right) \tau\left(h_{i j}\right)$, and the latter belongs to the set of finite sums of elements of $\sigma\left(G^{+}\right) \tau\left(H^{+}\right)$, and hence belongs to $\tau\left(H^{+}\right)$, there exist $h_{i}$ in $H^{+}$such that $\tau\left(h_{i}\right)=\sigma \otimes \tau\left(z_{i}\right)$, hence $\tau\left(h_{j}\right) \rightarrow 0$. Since $\tau$ is good, there exists $h_{i}^{\prime}$ in $H^{+}$such that $\tau\left(h_{i}\right)=\tau\left(h_{i}^{\prime}\right)$ and $\left\|\widehat{h}_{i}^{\prime}\right\|<2 \tau\left(h_{i}\right)$; thus $\widehat{h}_{i}^{\prime} \rightarrow 0$ uniformly, and it is immediate that if $w_{i}=u \otimes h_{i}^{\prime}$, then $\widehat{w}_{i} \rightarrow 0$ uniformly with respect to the affine representation of $(G \otimes H, u \otimes v)$. Obviously $\sigma \otimes \tau\left(w_{i}\right)=$ $\tau\left(h_{i}^{\prime}\right)=\tau\left(h_{i}\right)=\sigma \otimes \tau\left(z_{i}\right)$, and the density criterion is verified. Simplicity of the tensor product allows us to apply Corollary 1.8 .

Suppose in the preceding we change the order unit of $G$, say replacing it by $u^{\prime}$ where $\sigma\left(u^{\prime}\right)=\lambda$. Then $\sigma$ is replaced by $\sigma^{\prime}:=\sigma / \sigma\left(u^{\prime}\right)$, and of course goodness of $\sigma \otimes \tau$ is equivalent to that of $\sigma^{\prime} \otimes \tau$ (since goodness is defined without reference to any order unit). If $\sigma\left(G^{+}\right) \tau\left(H^{+}\right) / \lambda \subset \tau\left(H^{+}\right)$, then $\sigma^{\prime}\left(G^{+}\right) \tau\left(H^{+}\right) \subset \tau\left(H^{+}\right)$, whence (assuming $\tau$ is good) it follows that $\sigma^{\prime} \otimes \tau$ is good, and thus $\sigma \otimes \tau$ is good. Thus the condition can be weakened to the following, which simply says that $\tau(H)$ is order isomorphic to a module over the ring generated by $\sigma(G)$.

Corollary 5.4. Let $\sigma, \tau$ be normalized pure traces on the simple dimension groups with order unit, $(G, u),(H, v)$, respectively, and suppose moreover that $\tau$ is good, and $\sigma\left(G^{+}\right) \tau\left(H^{+}\right) \subseteq \lambda \tau\left(H^{+}\right)$for some real $\lambda>0$ in $\sigma\left(G^{+}\right)$. Then $\sigma \otimes \tau$ is a good pure trace of $G \otimes H$. 
There is a necessarily weak result in the opposite direction. If $J$ is a subgroup of $\mathbf{R}$ containing 1, let $F\langle J\rangle$ denote the smallest subfield of $\mathbf{R}$ containing $J$.

Lemma 5.5. Suppose $\sigma$ and $\tau$ are pure normalized traces on the simple dimension groups $(G, u)$ and $(H, v)$ respectively. Suppose that $\tau$ is bad (that is, $\operatorname{ker} \tau=\{0\}$ and $H$ has more than one trace), and satisfies the following additional property:

Whenever $\left\{\tau\left(h_{i}\right)\right\}$ is linearly independent over $\mathbf{Q}$ (for $\left.h_{i} \in H\right)$, then $\left\{\tau\left(h_{i}\right)\right\}$ is linearly independent over $F=F\langle\sigma(G)\rangle$.

Then $\sigma \otimes \tau$ is not good.

Proof. We show that $\operatorname{ker} \sigma \otimes \tau=(\operatorname{ker} \sigma) \otimes H$ - for which we may assume that $H$ is divisible. If $\tau^{\prime}$ is a normalized trace on $H$ distinct from $\tau$, then $\sigma \otimes \tau^{\prime}$ kills $\operatorname{ker} \sigma \otimes \tau$, and thus the latter cannot have dense image in $\{\sigma \otimes \tau\}^{\perp}$.

The argument is routine. Suppose $w=\sum_{i=1}^{l} g_{i} \otimes h_{i}$ is in $\operatorname{ker} \sigma \otimes \tau$, and $l$ is the least number of terms to represent $w$. If $l=1$, then $0=\sigma \otimes \tau(w)=\sigma\left(g_{1}\right) \tau\left(h_{1}\right)$; if $\tau\left(h_{1}\right)=0$, then $h_{1}=0$ and thus $w=0$; if $\sigma\left(g_{1}\right)=0$, then $w \in(\operatorname{ker} \sigma) \otimes H$.

If $l>1$, then $0=\sum_{i} \sigma\left(g_{i}\right) \tau\left(h_{i}\right)$. None of $\tau\left(h_{i}\right)$ can be zero (otherwise the corresponding $h_{i}$ would be zero, and we would obtain a shorter representation), and we claim $\left\{\tau\left(h_{i}\right)\right\}$ is linearly independent over $\mathbf{Q}$. If not, by relabelling there exist rational numbers $q_{i}$ such that $\tau\left(h_{l}\right)=\sum_{i=1}^{l-1} q_{i} \tau\left(h_{i}\right)$. Hence there exists a positive integer $N$ such that $N q_{i}$ are all integers and $\tau\left(N h_{l}-\sum\left(N q_{i}\right) h_{i}\right)=0$; as $\tau$ is one to one, this forces $N h_{l}=\sum_{i=1}^{l-1} N q_{i} h_{i}$. Then

$$
\begin{aligned}
N w & =g_{l} \otimes N h_{l}+\sum_{i=1}^{l-1} g_{i} \otimes N h_{i} \\
& =\sum_{i=1}^{l-1}\left(g_{i}+g_{l}\right) \otimes\left(\left(N+N q_{i}\right) h_{i}\right) .
\end{aligned}
$$

Since $H$ is divisible, we may find $j_{i}$ in $H$ such that $N j_{i}=\left(N+N q_{i}\right) h_{i}$, so that $w=\sum_{i=1}^{l-1}\left(g_{i}+g_{l}\right) \otimes j_{i}$, a representation with fewer terms. Hence $\left\{\tau\left(h_{i}\right)\right\}$ is linearly independent over $\mathbf{Q}$.

By hypothesis, $\left\{\tau\left(h_{i}\right)\right\}$ is linearly independent over $F$. However, $0=\sigma \otimes \tau(w)=$ $\sum \sigma\left(g_{i}\right) \tau\left(h_{i}\right)$. Since $\sigma\left(g_{i}\right)$ belongs to $F$, this forces all $\sigma\left(g_{i}\right)=0$, so that $w \in$ $($ ker $\sigma) \otimes H$.

The hypotheses are ridiculously strong. However, it is not clear how to significantly weaken them. For example, if the linear independence hypothesis is reduced to $F\langle\sigma(G)\rangle \cap F\langle\sigma(H)\rangle=\mathbf{Q}$, the result fails, as illustrated by the following example.

Example 8. A simple dimension group $(G, u)$ with a bad pure trace $\tau$ together with a subfield $E$ of the reals such that $F\langle\tau(G)\rangle \cap E=\mathbf{Q}$, but the pure trace $1 \otimes \tau$ of $E \otimes G$ is good.

Proof. Pick an irreducible cubic over the rationals with three real roots, $r, s, t$ for which $\mathbf{Q}[r]$ is not Galois; hence the splitting field must have Galois group $S_{3}$, and $s \notin \mathbf{Q}[r]$. Form the subgroup of $\mathbf{R}^{2}, G=\left\langle(1,1),(r, s),\left(r^{2}, s^{2}\right)\right\rangle$; this is free of rank three, and a dense subgroup of $\mathbf{R}^{2}$. Therefore, with the strict ordering, it is a dimension group (cf. [EHCLS80]; it can alternatively be described as $\mathbf{Z}[r]$ equipped with the inherited ordering from the field $\mathbf{Q}[r]$ induced by two of the three real embeddings). Since each of $\left\{1, r, r^{2}\right\}$ and $\left\{1, s, s^{2}\right\}$ is linearly independent over the 
rationals, both pure traces (arising from the coordinates) are bad. Let $\tau=\tau_{2}$, the projection on the second coordinate.

Now set $E=\mathbf{Q}[r]$ equipped with the total ordering inherited from $\mathbf{R}$. We note that $r+s+t \in \mathbf{Q}$, so that $\mathbf{Q}[r, s]$ is the splitting field, and its dimension is six. Hence $s$ satisfies a quadratic over $\mathbf{Q}[r]$. This immediately yields a nonzero kernel for $1 \otimes \tau_{2}$ on $E \otimes G$, and since the tensor product is a vector space over $\mathbf{Q}[r]$ (induced by the first factor), the kernel is a rational vector space, and therefore the only other trace, $1 \otimes \tau_{1}$, restricted to the kernel has dense range in $\mathbf{R}$, and therefore $1 \otimes \tau_{2}$ is good.

Not relevant to the hypotheses of the lemma, but it is immediate that $1 \otimes \tau_{1}$ is good, by Proposition 5.3.

Other combinations (for example, both $\tau$ and $\sigma$ not good, but $\sigma \otimes \tau$ good) are interesting, and almost anything can happen. As an easy example, suppose $G$ is a simple dimension group with two pure traces and no infinitesimals, and one of the traces, $\tau$, has the property that $\operatorname{ker} \tau$ is nonzero and cyclic. Then as we have seen, $\tau$ is not good. However, if $H=\mathbf{Q}$, then the induced trace $\tau \otimes 1$ has kernel $\mathbf{Q}$, and as there are no infinitesimals, and $G \otimes \mathbf{Q}$ still has just two pure traces, $\tau \otimes 1$ is good. In other words, tensoring with the rationals has improved $\tau$ to a good trace. However, if $\operatorname{ker} \tau=0$, then the kernel of $\tau \otimes 1$ is still zero, so $\tau \otimes 1$ is not good. We can arrange an example where $G$ is free of rank three with two pure traces, with one trace having nonzero kernel and the other not, so that one of the traces improves to good and the other one does not, on tensoring with the rationals.

More interestingly, we can easily construct an example of a simple dimension group $G$ such that none of its pure traces are good, but on $G \otimes G$ all of them are good. In this example (about the simplest possible), $G$ is free of rank three with two pure traces, so that $G \otimes G$ has $2 \times 2=4$ pure traces.

Example 9. A simple dimension group $G$ all of whose pure traces are ugly (and thus not good) such that all pure traces of $G \otimes G$ are good.

Let $G=\langle(0,1),(1,0),(\alpha, \beta)\rangle$ inside $\mathbf{R}^{2}$ with $\{1, \alpha, \beta\}$ linearly independent over the rationals; equipped with the strict ordering, this is Example 1 . Then $G$ is a simple dimension group, and its pure traces are the coordinate maps, call them $\tau_{1}$ and $\tau_{2}$. We already know that both traces are ugly. The four pure traces on $G \otimes G$ are $\left\{\tau_{i} \otimes \tau_{j}\right\}_{i, j=1,2}$.

We will identify $\mathbf{R}^{2} \otimes \mathbf{R}^{2}$ with $\mathbf{R}^{4}$, via $(a, b) \otimes(c, d) \mapsto(a c, a d, b c, b d)$. It is relatively easy to calculate enough of each $\operatorname{ker} \tau_{i} \otimes \tau_{j}$ to show that they are dense in the copy of $\mathbf{R}^{3}$ obtained by ignoring the entry corresponding to $(i, j)$.

With this identification, $G \otimes G$ is the subgroup of $\mathbf{R}^{4}$ generated by the following nine elements:

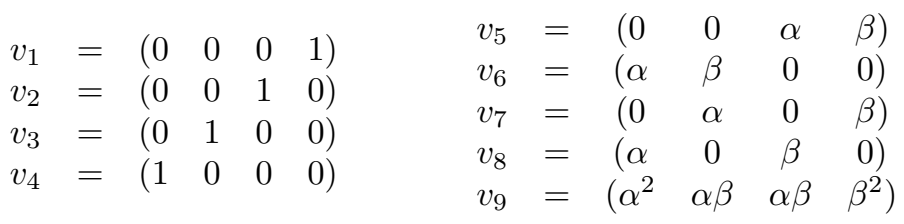

The four pure traces, denoted $\phi_{k}(k=1,2,3,4)$, are the coordinate projections. Just by looking at the locations of zeros, we see $\left\{v_{1}, v_{2}, v_{3}, v_{5}, v_{7}\right\} \subseteq \operatorname{ker} \phi_{1}$ (of course, $v_{8}-v_{6}$ also belongs, but we do not need it). Goodness will occur if this 
subgroup is dense in the copy of $\mathbf{R}^{3}$ obtained by deleting the first coordinate. The presence of the real basis $\left(\left\{v_{1}, v_{2}, v_{3}\right\}\right)$ makes density relatively easy to calculate.

The closure of the group span of $\left\{v_{1}, v_{2}, v_{5}\right\}$ is $0 \times \mathbf{R}^{2}$ (we ignore the leftmost coordinate, which is automatically zero). Hence $(\alpha, 0,0)$ (arising from $\left.v_{7}\right)$ is in the closure, and together with $v_{3}$, this yields $\mathbf{R} \times 0 \times 0$ in the closure of ker $\phi_{1}$. Since the closure of a group is a group, the closure is all of $\mathbf{R}^{3}$ and the trace $\phi_{1}$ is thus good.

For $\phi_{2}$, we have $\left\{v_{1}, v_{2}, v_{4}, v_{5}, v_{8}\right\} \subseteq \operatorname{ker} \phi_{2}$. Delete the second coordinate, and again we check density: $\left\{v_{2}, v_{4}, v_{8}\right\}$ generates a dense subgroup of $0 \times \mathbf{R}^{2}$, and using this and $v_{8}$ (and recalling that the closure of group is a group), we see that $(\alpha, 0,0)$ belongs to the closure of $\operatorname{ker} \phi_{2}$, and therefore (with $v_{1}$ ) $\mathbf{R} \times 0 \times 0$ contained in the closure, and again this means that the closure is all of $\mathbf{R}^{3}$.

Flipping the second and third coordinates, and then interchanging $\alpha$ with $\beta$ yields the same result for $\phi_{3}$ (from that for $\phi_{2}$ ). Similarly, flipping the first and fourth coordinates and interchanging $\alpha$ and $\beta$ yields the same for $\phi_{4}$ as that of $\phi_{1}$.

So all four pure traces of $G \otimes G$ are good. We did not even use $v_{9}$.

Still open: are all pure traces of $G \otimes G \otimes G$ good (same $G$ as in Example 9)? This is true if $\alpha$ is a cubic algebraic integer and $\beta=\alpha^{2}$, as we will see below. In other cases, it is not so clear.

Another choice for $G$ as free rank three and simple has $G=\left\langle(1,1),(r, s),\left(r^{2}, s^{2}\right)\right\rangle$, where $r$ and $s$ are two of three real roots of an irreducible cubic (so that $G$ is the ring of algebraic integers, $\mathbf{Z}[\alpha]$, where $\alpha$ satisfies a cubic with all roots real, with the ordering from two of the three real embeddings; this type of example was discussed in [EHCLS80, and later in Goo86] and Han13]). In this case, there is a symmetry that causes $\operatorname{ker} \phi_{1}=\operatorname{ker} \phi_{4}$ and $\operatorname{ker} \phi_{2}=\operatorname{ker} \phi_{3}$, so that none of the pure traces are $\operatorname{good}\left(\phi_{5-i}\right.$ vanishes on ker $\phi_{i}$, so density of the kernel in $\left\{\phi_{i}\right\}^{\perp}$ is impossible).

If $G$ is generated by three elements of $\mathbf{R}^{2}$ such that the set of six real numbers among its coordinates is algebraically independent, then all four traces of the tensor product have zero kernel, and so are all not good.

In Example 9, we can easily arrange $\sigma\left(G^{+}\right) \tau\left(G^{+}\right) \nsubseteq \tau(G)$ or $\sigma\left(G^{+}\right) \tau\left(G^{+}\right) \subseteq$ $\tau(G)$, as we wish, by ensuring respectively that $\{1, \alpha, \beta, \alpha \beta\}$ be linearly independent, or $\left\{\alpha^{2}, \beta^{2}, \alpha \beta\right\}$ be in the $\mathbf{Z}$-span of $\{1, \alpha, \beta\}$; e.g., $\alpha$ a cubic algebraic integer, and $\beta=\alpha^{2}$. If the last condition holds, then all pure traces of $G \otimes G \otimes G$ are good, since the condition in Proposition 5.3 is now satisfied.

Now we consider an interesting example arising from the tensor product of two polynomial rings with strict ordering inherited from points of $\mathbf{R}$. As in Example 5 , let $I$ and $J$ be compact subsets of $\mathbf{R}$ each having at least two points (typically $I$ and $J$ will be intervals, but need not be). Form the polynomial rings $G=\mathbf{Q}[x]$ and $H=\mathbf{Q}[y]$, and impose on them the strict ordering viewing the elements as functions on $I$ and $J$ respectively. Then each is a simple dimension group (as in Example 5), and their pure traces are the point evaluations $\left\{\sigma_{\alpha}\right\}_{\alpha \in I},\left\{\tau_{\beta}\right\}_{\beta \in J}$.

Example 10. Good pure traces on $\mathbf{Q}[x, y]$ with the strict ordering from $I \times J$, where $I$ and $J$ are compact subsets of $\mathbf{R}$.

The tensor product is naturally order and ring isomorphic to the polynomial ring in two variables, $\mathbf{Q}[x, y]$, equipped with the strict ordering from restricting to $I \times J$. To see this, the natural map $f \otimes g \mapsto f g$ extends to a ring isomorphism, it is positive, and the map induces a bijection on the pure traces. Since both $G \otimes H$ 
and $\mathbf{Q}[x, y]$ are simple, it follows that the map must be an order isomorphism. So we can identify $G \otimes H$ with $\mathbf{Q}[x, y]$ in the obvious way.

The pure traces of the latter must be multiplicative, and of course are exactly evaluations at points of $R=I \times J$ ( $R$ stands for rectangle, but neither $I$ nor $J$ need be intervals). For $z=(\alpha, \beta)$ in $I \times J$, let $\phi_{\alpha, \beta}$ be a point evaluation at $z$; of course, this corresponds to $\sigma_{\alpha} \otimes \tau_{\beta}$ after our identification.

We can ask when $\phi_{\alpha, \beta}$ is good. Since we are dealing with Bauer simplices here (taking the constant functions $\mathbf{1}$ as the order units for the respective dimension groups), the affine function spaces are respectively the continuous function rings $C(I, \mathbf{R}), C(J, \mathbf{R})$ and $C(R, \mathbf{R})$ equipped with the supremum norm, and $\left\{\sigma_{\alpha}\right\}^{\perp}$ is just the ideal of $C(I, \mathbf{R})$ consisting of continuous functions that vanish at $\alpha$, which we denote $\operatorname{Ann}(\alpha)$ (and similarly for $\left\{\tau_{\beta}\right\}^{\perp}$ and $\left\{\phi_{\alpha, \beta}\right\}^{\perp}$ ).

We have already determined when $\sigma_{\alpha}$ (respectively $\tau_{\beta}$ ) is a good trace: this occurs if and only if $\alpha$ is algebraic and no other roots of its (monic) irreducible polynomial over the rationals, $p_{\alpha}$, belong to $I$.

From the earlier result, for $(\alpha, \beta)$ in $R, \phi_{\alpha, \beta}$ is good if both $\sigma_{\alpha}$ and $\tau_{\beta}$ are good. We want to analyze the general case for points of $R$, but we do not obtain complete results. Nonetheless, interesting phenomena are going on. The rings $\mathbf{Q}[\alpha], \mathbf{Q}[\beta]$, and $\mathbf{Q}[\alpha, \beta]$ that appear are the rational subalgebras of the reals generated by the indicated elements. If both $\alpha$ and $\beta$ are algebraic, all three rings are subfields of R. Of course, $\sigma_{\alpha}(G)=\mathbf{Q}[\alpha], \tau_{\beta}(H)=\mathbf{Q}[\beta]$, and $\phi_{\alpha, \beta}(\mathbf{Q}[x, y])=\mathbf{Q}[\alpha, \beta]$.

- If $\phi_{\alpha, \beta}$ is good and $R$ is a rectangle (so $I$ and $J$ are intervals), and $(\alpha, \beta)$ belongs to the interior of $R$, then $\alpha$ and $\beta$ are algebraic over $\mathbf{Q}$.

Proof. If $\{\alpha, \beta\}$ is algebraically independent, then $\operatorname{ker} \phi_{\alpha, \beta}$ is $\{0\}$, contradicting $\phi_{\alpha, \beta}$ being good. By interchanging $x$ with $y$ if necessary, we may assume that $\alpha$ is transcendental, and $\beta$ is algebraic over the field $\mathbf{Q}(\alpha)$.

Let $f(x, y)=\sum_{j} y^{j} g_{j}(x)$ be the polynomial of minimal degree in $y$ with $g_{j} \in$ $\mathbf{Q}(x)$ such that $f(\alpha, \beta)=0$. Then $\operatorname{ker} \phi_{\alpha, \beta} \subseteq f \mathbf{Q}(x)[y]$. By multiplying by a suitable polynomial in $x$ (the product of the denominators of the $g_{j}$ ), we may assume that $g_{j}$ themselves are in $\mathbf{Q}[x]$, and moreover, since $\alpha$ is transcendental, this does not induce any new zeros on $(\alpha, y)$. (We subsequently use the fact that since $\alpha$ is transcendental, none of the denominators can vanish at $\alpha$; hence there exists a neighbourhood of $\alpha$ on which none of the denominators hit zero.)

We see that $f_{y}:=D_{y} f=\sum j y^{j-1} q_{j}(x)$, so by minimality of degree in $y$, $f_{y}(\alpha, \beta) \neq 0$. By the implicit function theorem [the first time we can recall using it!], there exists a continuous (actually analytic, but we don't require that) function $(x(t), y(t))$ on a neighbourhood of $\beta$ with $x(\beta)=\alpha$ such that $f(x(t), y(t))$ vanishes thereon (the leading term does not vanish on a neighbourhood of $\alpha$ either, take the intersection). Since $(\alpha, \beta)$ is in the interior of $R$, at least one point on the path other than $(\alpha, \beta)$, call it $\left(\alpha^{\prime}, \beta^{\prime}\right)$, will belong to $R$. Then $\operatorname{ker} \phi_{\alpha, \beta}$ will be killed by $\phi_{\alpha^{\prime}, \beta^{\prime}}$, since $f$ vanishes along the curve. Hence $\operatorname{ker} \phi_{\alpha, \beta}$ is not good.

This is a simplified version of the higher-dimensional case, which will be discussed later.

What happens on the boundary? As our colleague Damien Roy pointed out, the boundary of $I \times J$ (if $I$ and $J$ are intervals) can behave miserably. For example, if the upper left corner of $R$ is located at $(e, e)$ (where $e$ is transcendental), then ker $\phi_{e, e}=(x-y) \mathbf{Q}[x, y]$, and since the line $x=y$ hits $R$ only at $(e, e)$, it follows 
that the closure of $\operatorname{ker} \phi_{e, e}$ in $C(R, \mathbf{R})$ is $(e, e)^{\perp}$, hence $\phi_{e, e}$ is good. This is the first example wherein both $\sigma$ and $\tau$ have zero kernel (are bad), but $\sigma \otimes \tau$ is good. This can also occur on an edge; e.g., if $\left(e, e^{2}\right)$ is a point on the upper horizontal edge of $R$, then ker $\phi_{e, e^{2}}$ is $\left(x^{2}-y\right) \mathbf{Q}[x, y]$, and the zero set of $x^{2}-y$ has intersection with $R$ consisting only of the point $\left(e, e^{2}\right)$, and thus $\phi_{e, e^{2}}$ is good.

In contrast, $\phi_{\alpha, \beta}$ is bad if and only if $\{\alpha, \beta\}$ is algebraically independent.

Because of this pathology on the boundary, we assume from now on that $\alpha$ and $\beta$ are algebraic over $\mathbf{Q}$.

- If $\mathbf{Q}[\alpha] \subseteq \mathbf{Q}[\beta]$ and $\tau_{\beta}$ is good, then $\phi_{\alpha, \beta}$ is good. This is an immediate consequence of Proposition 5.3.

In particular, if $\mathbf{Q}[\alpha]=\mathbf{Q}[\beta]$, then sufficient for $\phi_{\alpha, \beta}$ to be good is that either $\sigma_{\alpha}$ or $\tau_{\beta}$ be good. We will improve on this, but before doing so, we obtain an easy result.

If $K$ and $L$ are subfields of a field $M$ with each containing a subfield $N$, we say they are disjoint (with respect to $N$ ) if $K \otimes_{N} L$ is naturally isomorphic to $K L$, the field they generate inside $M$; equivalently, $\operatorname{dim}_{N} K L=\left(\operatorname{dim}_{N} K\right)\left(\operatorname{dim}_{N} L\right)$ (and there are other equivalent formulations; for example, $K \otimes_{N} L$ is a field). In our case, we will restrict to $N=\mathbf{Q}, K=\mathbf{Q}[\alpha]$, and $L=\mathbf{Q}[\beta]$; then $K L=\mathbf{Q}[\alpha, \beta]$, and disjointness occurs if and only if $p_{\beta}$ (the irreducible polynomial of $\beta$ over $\mathbf{Q}$ ) is irreducible over $\mathbf{Q}[\alpha]$. This is stronger than merely $K \cap L=\mathbf{Q}$ (in fact, $K$ and $L$ could be unequal but isomorphic, in which case their tensor product will not be a field). Disjointness is very closely related to the property discussed in Lemma 5.5.

- Assume that $\mathbf{Q}[\alpha]$ and $\mathbf{Q}[\beta]$ are disjoint; then $\phi_{\alpha, \beta}$ is good if and only if both $\sigma_{\alpha}$ and $\tau_{\beta}$ are good.

Proof. Let $\mathcal{I}$ be the ideal of $\mathbf{Q}[x, y]$ generated by $\left\{p_{\alpha}(x), p_{\beta}(y)\right\}$ (that is, the polynomials in $\mathbf{Q}[x]$ and $\mathbf{Q}[y]$ that are the monic irreducible polynomials of $\alpha$, respectively $\beta$, over $\mathbf{Q}$ ). Then $\mathcal{I}$ is contained in $\operatorname{ker} \phi_{\alpha, \beta}$, and obviously $\mathbf{Q}[x, y] / \mathcal{I}$ has dimension $\left(\operatorname{deg} p_{\alpha}\right)\left(\operatorname{deg} p_{\beta}\right)$; however, this is the same as $\operatorname{dim}_{\mathbf{Q}} \mathbf{Q}[\alpha, \beta]$, the image of $\phi_{\alpha, \beta}$. Thus, $\mathcal{I}=\operatorname{ker} \phi_{\alpha, \beta}$.

If $\phi_{\alpha}$ were not a good trace of $G=\mathbf{Q}[x]$, then $p_{\alpha}$ would have another root, $\alpha^{\prime}$, in $\mathcal{I}$. Then $\phi_{\alpha^{\prime}, \beta}$ kills $\mathcal{I}$, and therefore $\mathcal{I}$ cannot be dense in $(\alpha, \beta)^{\perp}$, so that $\phi_{\alpha}$ is not good. Similarly, $p_{\beta}$ not good implies $\phi_{\alpha, \beta}$ is not good. The converse is a consequence of 5.2 .

- General criterion. If $\alpha$ and $\beta$ are both algebraic over $\mathbf{Q}$, and $p(x, y)$ is such that $p(\alpha, y)$ is the monic irreducible polynomial of $\beta$ over $\mathbf{Q}[\alpha]$, then $\operatorname{ker} \phi_{\alpha, \beta}=\left(p_{\alpha}(x), p(x, y)\right)$. In particular, $\phi_{\alpha, \beta}$ is good if and only if for every algebraic conjugate $\alpha^{\prime}$ of $\alpha$ in $I$, and every algebraic conjugate $\beta^{\prime}$ of $\beta$ over $\mathbf{Q}[\alpha]$ in $J, p\left(\alpha^{\prime}, \beta^{\prime}\right)=0$ implies $\left(\alpha^{\prime}, \beta^{\prime}\right)=(\alpha, \beta)$.

This is a consequence of a more general result (obtained later) about generating sets for annihilating ideals, but is too difficult to work with, so we consider a number of special cases.

Now we give sufficient conditions for $\phi_{\alpha, \beta}$ to be good; these are also necessary when $\mathbf{Q}[\alpha] \subseteq \mathbf{Q}[\beta]$ (still assuming they are algebraic). We will see interesting phenomena after we cut through the technicalities.

If $f$ is a polynomial in one variable (either $x$ or $y$ ), $Z(f)$ will denote the set of its roots in $\mathbf{C}$. Suppose that $K:=\mathbf{Q}[\alpha] \cap \mathbf{Q}[\beta]$ is nontrivial; that is, the intersection is not the rationals. By the primitive element theorem, there exists $\theta$ in $K$ such 
that $K=\mathbf{Q}[\theta]$. We can express $\theta$ uniquely in each of the forms, $\theta=\sum_{i=0}^{s} q_{i} \alpha^{i}$ and $\theta=\sum_{j=0}^{t} r_{j} \beta^{j}$, where $q_{i}$ and $r_{j}$ are all rational, $q_{s} r_{t} \neq 0, s<\operatorname{deg} p_{\alpha}$, and $t<\operatorname{deg} p_{\beta}$. Set $f \equiv f_{\theta}=\sum q_{i} x^{i}$ and $h \equiv h_{\theta}=\sum r_{j} y^{j}$. Then $f \in \mathbf{Q}[x] \subset \mathbf{Q}[x, y]$, $h \in \mathbf{Q}[y] \subset \mathbf{Q}[x, y]$, and $f(\alpha)=\theta=h(\beta)$. Let $\mathcal{I}$ denote the ideal of $\mathbf{Q}[x, y]$ generated by $\left\{p_{\alpha(x)}, p_{\beta}(y), f(x)-h(y)\right\}(\mathcal{I}$ sometimes depends on the choice of $\theta)$. Then $\mathcal{I}$ is contained in $\operatorname{ker} \phi_{\alpha, \beta}$, frequently equalling it.

Lemma 5.6. Sufficient for $\mathcal{I}=\left(p_{\alpha}(x), p_{\beta}(y), f(x)-h(y)\right)$ to be dense in $(\alpha, \beta)^{\perp}$ is the following set of conditions:

(i) $f^{-1}(f(\alpha)) \cap Z\left(p_{\alpha}\right) \cap I=\{\alpha\}$;

(ii) $h^{-1}(h(\beta)) \cap Z\left(p_{\beta}\right) \cap J=\{\beta\}$;

(iii) $f\left(Z\left(p_{\alpha}\right) \cap I\right) \cap h\left(Z\left(p_{\beta}\right) \cap J\right)=\{\theta\}=\{f(\alpha)\}$.

These guarantee that $\phi_{\alpha, \beta}$ is good. When $\mathcal{I}=\operatorname{ker} \phi_{\alpha, \beta}$, these conditions are also necessary for $\phi_{\alpha, \beta}$ to be good.

Remark. All the conditions refer to the behaviour of finite sets (the zeros of $p_{\alpha}$ in $I$ and the zeros of $p_{\beta}$ in $J$ ) under $f$ and $h$ respectively. They simplify drastically in special cases.

Proof. We shall prove density of $\mathcal{I}$ in $(\alpha, \beta)^{\perp}$; this will force density of ker $\phi_{\alpha, \beta}$, which in turn yields that $\phi_{\alpha, \beta}$ is good. Let $\overline{\mathcal{I}}$ denote the closure of $\mathcal{I}$ in $C(R, \mathbf{R})$. Since $\mathcal{I}$ is an ideal of $\mathbf{Q}[x, y]$ contained in $\operatorname{ker} \phi_{\alpha, \beta}, \overline{\mathcal{I}}$ is a closed ideal of $C(R, \mathbf{R})$, obviously contained in $(\alpha, \beta)^{\perp}$. Hence to show equality, it suffices to show that for all points $v$ of $R \backslash(\alpha, \beta)$, evaluation at $v$ does not kill $\mathcal{I}$.

Write $v=(\gamma, \delta) \in R=I \times J$, and suppose $\phi_{\gamma, \delta}(\mathcal{I})=\{0\}$. Evaluating the generators, $p_{\alpha}(x), p_{\beta}(y)$, and $f(x)-h(y)$ at $v$, we obtain (a) $\gamma \in Z\left(p_{\alpha}\right) \cap I$, (b) $\delta \in Z\left(p_{\beta}\right) \cap J$, and $(\mathrm{c}) f(\gamma)=h(\delta)$.

If $\gamma \neq \alpha$ but $\delta=\beta$, we deduce $f(\gamma)=f(\alpha)$, contradicting (i). If $\gamma=\alpha$ and $\delta \neq \beta$, then $h(\delta)=h(\beta)$, contradicting (ii).

If $\gamma \neq \alpha$ and $\delta \neq \beta$, we note by (i) and (ii) that $f(\gamma) \neq f(\alpha)=h(\beta) \neq h(\delta)$, and $f(\gamma)=h(\delta)$ contradicts (iii). Hence $\mathcal{I}$ is dense in $\phi_{\alpha, \beta}$.

Now suppose $\mathcal{I}=\operatorname{ker} \phi_{\alpha, \beta}$ and at least one of (i)-(iii) is violated. We will find $v$ in $R \backslash\{(\alpha, \beta)\}$ such that evaluation at $v$ kills $\mathcal{I}$; hence $\operatorname{ker} \phi_{\alpha, \beta}$ is not dense, so by Proposition 1.7, $\phi_{\alpha, \beta}$ is not good. If (i) fails, there exists $\alpha^{\prime}$ in $Z\left(p_{\alpha}\right) \cap I$ such that $\alpha^{\prime} \neq \alpha$ and $f\left(\alpha^{\prime}\right)=f(\alpha)=h(\beta)$. Set $v=\left(\alpha^{\prime}, \beta\right) \in I \times J$; obviously, $\phi_{\alpha^{\prime}, \beta}\left(p_{\alpha}(x)\right)=\phi_{\alpha^{\prime}, \beta}\left(p_{\beta}(y)\right)=0$, and $\phi_{\alpha^{\prime}, \beta}(f(x)-h(y))=f\left(\alpha^{\prime}\right)-h(\beta)=0$, so $\phi_{\alpha^{\prime}, \beta}$ kills $\mathcal{I}$.

If (ii) fails, there exists $\beta^{\prime}$ in $Z\left(p_{\beta}\right) \cap J$, unequal to $\beta$, such that $h\left(\beta^{\prime}\right)=h(\beta)$; set $v=\left(\alpha, \beta^{\prime}\right) \in I \times J$, and as in the previous case, we see that $\phi_{\alpha, \beta^{\prime}}$ kills $\mathcal{I}$.

If (iii) fails, there exist $\alpha^{\prime}$ in $Z\left(p_{\alpha}\right) \cap I$ and $\beta^{\prime}$ in $Z\left(p_{\beta}\right) \cap J$ such that $f\left(\alpha^{\prime}\right)=h\left(\beta^{\prime}\right)$. Then $v=\left(\alpha^{\prime}, \beta^{\prime}\right)$ is the desired point.

The ideal $\mathcal{I}$ depends on the choice of $\theta$, and it might be useful to examine the larger ideal obtained by considering all choices of $\theta$. However, still left unconsidered are the cases wherein $\mathbf{Q}[\alpha] \cap \mathbf{Q}[\beta]=\mathbf{Q}$ and the two fields are not disjoint.

Corollary 5.7. Suppose $\mathbf{Q} \neq \mathbf{Q}[\alpha] \subseteq \mathbf{Q}[\beta]$; with unique choice of $h$ of degree less than $\operatorname{deg} p_{\beta}$ such that $\alpha=h(\beta)$, define $\mathcal{I}$ to be the $\mathbf{Q}[x, y]$-ideal generated by $\left\{p_{\alpha}(x), p_{\beta}(y), x-h(y)\right\}$. Then $\mathcal{I}=\operatorname{ker} \phi_{\alpha, \beta}$. Moreover, $\phi_{\alpha, \beta}$ is good if and only if for all $\beta^{\prime} \neq \beta$ in $Z\left(p_{\beta}\right) \cap J$, (ii E iii) $h\left(\beta^{\prime}\right) \notin Z\left(p_{\alpha}\right) \cap I$. 
Proof. We observe that the codimension of $\mathcal{I}$ is at most $\operatorname{deg} p_{\beta}$, but this is the dimension of $\mathbf{Q}[\beta]=\mathbf{Q}[\alpha, \beta]=\phi_{\alpha, \beta}(\mathbf{Q}[x, y])$; since $\mathcal{I} \subseteq \operatorname{ker} \phi_{\alpha, \beta}$, equality holds.

Now condition (i) of the previous result is automatic, and (ii) and (iii) translate to the combined condition hypothesized.

There is one more easy case: if $\alpha$ is rational (that is, $\mathbf{Q}[\alpha]=\mathbf{Q}$ ); in particular, $\sigma_{\alpha}$ is good. Then $\operatorname{ker} \phi_{\alpha, \beta}$ is the ideal generated by $\left\{x-\alpha, p_{\beta}(y)\right\}$. Now it is obvious that $\phi_{\alpha, \beta}$ is good if and only if $\phi_{\beta}$ is. This is also covered by the disjointness result.

Corollary 5.8. (a) Suppose that $\alpha=\beta^{k}$ for some positive integer $k<\operatorname{deg} p_{\beta}$. Then $\phi_{\alpha, \beta}$ is good if and only if no kth power of a root in $J$ of $p_{\beta}$ other than $\beta$ equals a root of $p_{\alpha}$ in $I$, and if $k$ is even, $-\beta$ is not in $J \cap Z\left(p_{\beta}\right)$.

(b) If $\alpha \in I \cap J$, then $\phi_{\alpha, \alpha}$ is good if and only if $I \cap J \cap Z\left(p_{\alpha}\right)=\{\alpha\}$.

(c) If $\alpha / \beta=q \in \mathbf{Q} \backslash\{0\}$, then $\phi_{\alpha, \beta}$ is good if and only if $q^{-1} I \cap J \cap Z\left(p_{\beta}\right)=\{\beta\}$.

Proof. We verify conditions (ii \& iii) of Corollary 5.7. (a) Here $h(y)=y^{k}$, so $h(y)=\alpha$ has only nonreal roots (other than $\beta$ ) if $k$ is odd; otherwise it has $-\beta$ as a zero. (b) Here $h(y)=y$. (c) Here $h(y)=q y$.

This allows us to construct many examples. For instance (always assuming $\alpha$ is algebraic), suppose $\alpha \in I \cap J$, and none of its other algebraic conjugates belong to $I \cap J$, but $Z\left(p_{\alpha}\right) \cap I$ and $Z\left(p_{\alpha}\right) \cap J$ both contain at least two elements (one of which is $\alpha$ ). Then neither $\sigma_{\alpha}$ nor $\tau_{\alpha}$ is good, but their tensor product, $\phi_{\alpha, \alpha}$, is good. Specifically, we can arrange this by setting $I=[a, b], J=[c, d]$, and $p$ an irreducible rational polynomial with at least three real roots, $\alpha_{2}<\alpha<\alpha_{1}$ with $\alpha_{2}<a<\alpha<\alpha_{1}<b$ and $c<\alpha_{2}<\alpha<d<\alpha_{1}$, such that all other real roots are either bigger than $b$ or less than $c$.

For example, the irreducible polynomial of $\alpha=\sqrt{2}+\sqrt{3}$ is degree four, and the four roots, all real, are $\pm \sqrt{2} \pm \sqrt{3}$. By varying the locations of $a \leq \alpha \leq b$ and $c \leq \alpha \leq d$, various phenomena can be observed.

Corollary 5.9. Suppose $\tau_{\beta}$ is good. Then $\phi_{\alpha, \beta}$ is good if $f\left(\alpha^{\prime}\right) \neq f(\alpha)$ for all $\alpha^{\prime} \neq \alpha$ in $Z\left(p_{\alpha}\right) \cap I$.

Proof. We have already seen that $\tau_{\beta}$ is good if and only if $Z\left(p_{\beta}\right) \cap J$ is the singleton $\{\beta\}$. Thus conditions (ii) and (iii) are automatically satisfied, and the hypothesized condition is a reformulation of (i).

This example suggests more examples. Let $M$ be a compact subset of $\mathbf{R}^{n}$, preferably regular (equalling the closure of its interior), but not necessarily. Form $A=\mathbf{Q}\left[x_{1}, \ldots, x_{n}\right]$; viewed as a ring of functions on $M, A$ will be norm dense in $C(M, \mathbf{R})$, so equipped with the strict ordering, $A$ is a simple dimension group and also an ordered ring. For $\alpha=\left(\alpha_{1}, \ldots, \alpha_{n}\right)$ in $M$, define the point evaluation at $\alpha$, $\phi_{\alpha}$; as before, this is a pure trace of $A$, and they are all of this form.

Then $\phi_{\alpha}$ will be a good trace if and only if the zero set of ker $\phi_{\alpha}$ contains no points of $M$ other than $\alpha$ (this comes from the density criterion). If we use the notation $I_{\mathbf{Q}}(S)$ for the ideal of $\mathbf{Q}\left[x_{1}, \ldots, x_{n}\right]$ consisting of those elements that vanish at a subset $S$ of $M$ (of course, if $F$ is a subfield of the complexes, then we can define $I_{F}$ similarly, as an ideal in $\left.F\left[x_{1}, \ldots, x_{n}\right]\right)$, then $\phi_{\alpha}$ is good if and only if

$$
Z\left(I_{\mathbf{Q}}(\alpha)\right) \cap M=\{\alpha\}
$$


(recall that for $U$ a set of polynomials, $Z(U)$ denotes the set of complex zeros common to all elements of $U$ ).

Modulo the usual obnoxious boundary behaviour, we have a consequence of the implicit function theorem, again.

Proposition 5.10. Let $M$ be a compact subset of $\mathbf{R}^{n}$, and let $A=\mathbf{Q}\left[x_{1}, \ldots, x_{n}\right]$ equipped with the strict ordering as functions on $M$. Suppose that $\alpha=\left(\alpha_{1}, \ldots, \alpha_{n}\right)$ is in the interior of $M$. If the pure trace of $A, \phi_{\alpha}$ (evaluation at $\alpha$ ) is good, then all $\alpha_{i}$ are algebraic over the rationals.

This requires the following well known result ([Mum76, Chapter I]) on generating sets for annihilator ideals.

Proposition 5.11. Let $K$ be a subfield of $\mathbf{R}$, and let $b=\left(\beta_{1}, \ldots, \beta_{s}\right)$ be a point in $\mathbf{R}^{s}$ all of whose entries are algebraic over $K$. There exist polynomials $\left\{f_{i}\right\}_{i=1}^{s}$ of the polynomial ring $K\left[y_{1}, \ldots, y_{s}\right]$ with the following properties:

(i) $f_{i} \in K\left[y_{1}, y_{2}, \ldots, y_{i}\right]$ for all $1 \leq i \leq s$;

(ii) $\frac{\partial f_{i}}{\partial y_{i}}(b) \neq 0$ for all $1 \leq i \leq s$;

(iii) $I_{K}(b)$ is the ideal of $K\left[y_{1}, \ldots, y_{s}\right]$ generated by $\left\{f_{1}, \ldots, f_{s}\right\}$.

Proof. Define the following polynomials. $F_{i} \in K\left[\beta_{1}, \ldots, \beta_{i-1}\right]\left[y_{i}\right]$ (so $\left.F_{1} \in K\left[y_{1}\right]\right)$, via $F_{i}$, is the monic irreducible polynomial, in $y_{i}$, of $\beta_{i}$ over $K\left[\beta_{1}, \ldots, \beta_{i-1}\right]$. Now define $f_{i} \in K\left[y_{1}, \ldots, y_{i}\right]$ by replacing every occurrence of $\beta_{j}$ in $F_{i}$ by $y_{j}$ (for every $j<i$ ); we may also assume (by using the algebraicity of $\beta_{j}$ ) that the degree of $y_{j}$ in $f_{i}$ is at most $K\left[\beta_{1}, \ldots, \beta_{j}\right]: K\left[\beta_{1}, \ldots, \beta_{j-1}\right]-1$. (This last is not necessary for the argument here, but would be useful if we made it into an algorithm.) Then $f_{i}\left(\beta_{1}, \ldots, \beta_{i-1}, y_{i}\right)=F_{i}\left(y_{i}\right)$.

Each $f_{i}$ is in $K\left[y_{1}, \ldots, y_{i}\right] \subseteq K\left[y_{1}, \ldots, y_{s}\right]$, so we can evaluate them at points of $\mathbf{R}^{s}$. Let $b^{(i)}$ be the truncation to the first $i$ coordinates. Then $f_{i}(b)=F_{i}\left(b^{(i)}\right)=0$. In particular, $f_{i} \in I_{K}(b)$.

Let $h \in K\left[y_{1}, \ldots, y_{s}\right]$ be such that $h(b)=0$. Then $h_{s}\left(y_{s}\right):=h\left(b^{(i-1)}, y_{s}\right)$ vanishes at $y_{s}=\beta_{s}$, so that as elements of $K\left[\beta_{1}, \ldots, \beta_{s-1}\right]\left[y_{s}\right], F_{s}$ divides $h_{s}$. That is, there exists $G_{s} \in K\left[\beta_{1}, \ldots, \beta_{s-1}\right]$ such that $h_{s}=F_{s} G_{s}$. We may form $g_{s}$ in $K\left[y_{1}, \ldots, y_{s}\right]$ as before, so that $G_{s}\left(y_{s}\right)=g_{s}\left(b^{(s-1)}, y_{s}\right)$.

Now form $h^{(s-1)}=h_{s-1}\left(y_{s-1}, y_{s}\right)-f_{s}\left(b^{s-2}, y_{s-1}, y_{s-1}, y_{s}\right) g_{s}\left(b^{(s-2)}, y_{s-1}, y_{s}\right)$. We can rewrite this as $\sum y_{s}^{l} \sum c_{l}\left(y_{s-1}\right)$, where $c_{l}$ belongs to $K\left[\beta_{1}, \ldots, \beta_{s-2}\right]\left[y_{s-1}\right]$. Plugging in $y_{s-1}=\beta_{s-1}$, we obtain that each $c_{l}\left(\beta_{s-1}\right)$ must be zero (since the identity is true for all values of $y_{s}$ ), hence $F_{s-1}$ divides each one of those. Thus we have $h^{(s-1)}\left(y_{s-1}, y_{s}\right)=F_{s-1} G_{s-1}$, where $G_{s-1}$ belongs to $K\left[\beta_{1}, \ldots, \beta_{s-2}, y_{s-1}, y_{s-2}\right]$. As before, we can find $g_{s-1}$ with coefficients in $K\left[y_{1}, \ldots, y_{s}\right]$ such that

$$
g_{s-1}\left(b^{s-2}, y_{s-2}, y_{s-1}\right)=G_{s-1} .
$$

Then

$$
\begin{aligned}
h\left(b^{(s-2)}, y_{s-1}, y_{s}\right) & -f_{s}\left(b^{(s-2)}, y_{s-1}, y_{s-1}, y_{s}\right) g_{s}\left(b^{(s-2)}, y_{s-1}, y_{s}\right) \\
& -f_{s-1}\left(b^{(s-2)}, y_{s-1}, y_{s}\right) g_{s-1}\left(b^{(s-2)}, y_{s-1}, y_{s}\right)
\end{aligned}
$$

vanishes identically. 
We can continue this process, obtaining successively $g_{s-j} \in K\left[y_{1}, \ldots, y_{s}\right](j=$ $0,1,2, \ldots)$ such that

$$
\begin{aligned}
& h\left(b^{(s-j-1)}, y_{s-j}, \ldots, y_{s}\right) \\
& \quad-\sum_{k=0}^{j-1} f_{s-k}\left(b^{(s-k-1)}, y_{s-k}, \ldots, y_{s-j}\right) g_{s-k}\left(b^{(s-k-1)}, y_{s-k}, \ldots, y_{s}\right)
\end{aligned}
$$

vanishes identically. This continues until we run out of coordinates, and we obtain $h=\sum f_{s-j} g_{s-j}$. This yields (iii); (i) is by construction, and (ii) follows from the fact that $\frac{d F_{i}}{d y_{i}}\left(\beta_{i}\right) \neq 0$, as the irreducible polynomial, $F_{i}\left(y_{i}\right)$, has no repeated roots.

Proof of of Proposition 5.10. If $\left\{\alpha_{1}, \ldots, \alpha_{n}\right\}$ is algebraically independent, then ker $\phi_{\alpha}=\{0\}$, so $\phi_{\alpha}$ could not be good. Hence after relabelling, we may assume that for some $r$ with $1 \leq r<n$, the set $\left\{\alpha_{1}, \ldots, \alpha_{r}\right\}$ is algebraically independent (over $\mathbf{Q}$ ), and each of $\beta_{k}:=\alpha_{r+k}(k>0)$ is algebraic over the function field, $K=\mathbf{Q}\left(\alpha_{1}, \ldots, \alpha_{r}\right)$. Let $a=\left(\alpha_{1}, \ldots, \alpha_{r}\right)$. We may find the polynomials (with coefficients from $K$ ) $f_{i}$ satisfying the conditions above (where $y_{i}:=x_{r+i}$ ). We can thus write each

$$
f_{i}:=\sum y^{w} \frac{A_{w i}}{B_{w i}}
$$

where $w$ is an $i$-tuple in $\left(\mathbf{Z}^{+}\right)^{i}$ (and $y^{w}$ is the corresponding monomial in the $y$ 's), $A_{w i}$ and $B_{w i}$ are elements of $\mathbf{Q}\left[\alpha_{1}, \ldots, \alpha_{r}\right]$ such that none of $B_{w i}$ are zero, and at least one of $A_{w i}$ is not zero. Because $a$ is algebraically independent, there exist unique $a_{w i}, b_{w i}$ in $\mathbf{Q}\left[x_{1}, \ldots, x_{r}\right]$ such that $a_{w i}(a)=A_{w i}$ and $b_{w i}(a)=B_{w i}$; set $f_{i}^{0}$ to be the resulting elements of $\mathbf{Q}\left(x_{1}, \ldots, x_{r}\right)\left[y_{1}, \ldots, y_{s}\right]$. Set $q=\prod_{w, i} b_{w i}$. Since $a$ is algebraically independent, $q(a) \neq 0$. Hence there exists a neighbourhood, $N$, of $a$ in $\mathbf{R}^{r}$ such that $q$ has no zeros in $N \times \mathbf{R}^{n-r}$; of course, each $q f_{i}^{0}$ belongs to $\mathbf{Q}\left[x_{1}, \ldots, x_{r} ; y_{1}, \ldots, y_{i}\right]$. It easily follows that if $h \in \mathbf{Q}\left[x_{1}, \ldots, x_{r}, y_{1}, \ldots, y_{s}\right]$ and $h(a, b)=0$, then there exists $p \in \mathbf{Q}\left[x_{1}, \ldots, x_{r}\right]$, with no zeros on $N$, such that $p h$ is in the ideal of $\mathbf{Q}\left[x_{1}, \ldots, x_{r}, y_{1}, \ldots, y_{s}\right]$ generated by $\left\{q f_{i}^{0}\right\}$.

Define the map $\left(q f_{i}^{0}\right): N \times \mathbf{R}^{s} \rightarrow \mathbf{R}^{s}$ by $v \mapsto\left(q f_{1}^{0}(v), q f_{2}^{0}(v), \ldots, q f_{s}^{0}(v)\right)$. This is analytic on $N \times R^{s}$ and sends $(a, b)$ to $\mathbf{0}$.

Calculating the Jacobian matrix of the map, $J=\left(\frac{\partial q f_{i}^{0}}{\partial y_{j}}\right)$, we see that $J$ is an upper diagonal matrix, whose entries on the diagonal do not vanish at $(a, b)$. Hence $J(a, B)$ is invertible, so we may apply the implicit function theorem. There exist an open neighbourhood $V \subseteq N$ in $\mathbf{R}^{r}$ of $a$, an open neighbourhood $W$ of $b$ in $\mathbf{R}^{s}$, together with an analytic function $g: V \rightarrow W$ with $g(a)=b$ and such that the graph of $g$ is $\left\{(v, w) \in V \times W \mid\left(q f_{i}^{0}\right)(v, w)=\mathbf{0}\right\}$.

Since $\alpha$ is in the interior of $M$, there exists a point in $M$ of the form $\left(a^{\prime}, b^{\prime}\right):=$ $\left(\alpha_{1}^{\prime}, \ldots, \alpha_{r}^{\prime}, g\left(\alpha_{1}^{\prime}, \ldots, \alpha_{r}^{\prime}\right)\right)$ unequal to $(a, b)$. Hence $f_{i}^{0}\left(a^{\prime}, b^{\prime}\right)=0$ for all $i$. If $h$ in $\mathbf{Q}\left[x_{i}, y_{j}\right]$ vanishes at $(a, b)$, we saw previously that $p h$ vanishes where all the $f_{i}^{0}$ do, and since $\left(a^{\prime}, b^{\prime}\right)$ is in $N \times \mathbf{R}^{s}, p$ vanishes nowhere on this neighbourhood, and thus $h\left(a^{\prime}, b^{\prime}\right)=0$. In particular, $\phi_{a^{\prime}, b^{\prime}}$ kills ker $\phi_{a, b}$, hence $\phi_{a, b}$ is not good.

The argument proves a bit more; since there is at least a curve of zeros in the neighbourhood, $Z\left(I_{\mathbf{Q}}(\alpha)\right) \cap M$ contains a continuum when not all the entries of $\alpha$ are algebraic and $\alpha$ is in the interior of $M$ - so there are uncountably many pure traces that kill ker $\phi_{\alpha}$. 
One consequence of the existence of the generating set is that if $\alpha=\left(\alpha_{i}\right)$ is an algebraic point, then the closure of $\operatorname{ker} \phi_{\alpha}$ in $C(M, \mathbf{R})$ is of codimension at most $t:=\operatorname{dim}_{\mathbf{Q}} \mathbf{Q}\left[\alpha_{1}, \ldots, \alpha_{n}\right]-1$ in the ideal Ann $(\alpha)$ of $C(M, \mathbf{R})$. Another aspect is that we need only check the generating set on at most $t$ points to decide whether $\phi_{\alpha}$ is good.

The problem with the boundary would go away in the weird case that for every nonalgebraic boundary point of $M$, there are no analytic curves whose intersection with $M$ consists locally exactly of the boundary point.

\section{IMPURE TRACES}

Some results on goodness require $\tau$ to be pure. If $\tau$ is not pure, then it might still be worthwhile to study goodness, because it requires some relations among the pure traces in the support.

Lemma 6.1. Let $G$ be an approximately divisible dimension group with order unit, and let $\tau_{1}$ and $\tau_{2}$ be pure normalized traces. Let $\lambda$ be a real number in the open unit interval, and define $\tau=\lambda \tau_{1}+(1-\lambda) \tau_{2}$. If $\lambda \tau_{1}(G) \cap(1-\lambda) \tau_{2}(G)=\{0\}$, then $\tau$ is not good.

Remark. If $G$ is countable, then the set of $\lambda$ for which $\lambda \tau_{1}(G) \cap(1-\lambda) \tau_{2}(G)=\{0\}$ is cocountable, so that goodness hardly ever occurs for these combinations.

Remark. As is clear from the proof, $\tau_{i}$ need not be pure, but simply different enough that they can be separated by elements of the affine function space.

Proof. Select positive real numbers $r$ and $s$ such that $r>\lambda s /(1-\lambda)$. There exists $h$ in the affine function space such that $h\left(\tau_{1}\right)=-s$ and $h\left(\tau_{2}\right)=r$. For all sufficiently small $\epsilon$, perturbations of $r$ and $s$ independently, by at most $\epsilon$ will guarantee that the perturbed $-s$ is negative, the perturbed $r$ is still positive, and the perturbed $r$ and $s$ will still satisfy the inequality $r^{\prime}>\lambda s^{\prime} /(1-\lambda)$. As $G$ is approximately divisible, we may find $g$ in $G$ such that $\|\widehat{g}-h\|<\epsilon$. Then $\left|\tau_{1}(g)+s\right|,\left|\tau_{2}(g)-r\right|<\epsilon$ (so $\tau_{1}(g)<0$ and $\tau_{2}(g)>0$ ) and

$$
\tau_{2}(g)>-\frac{\lambda \tau_{1}(g)}{1-\lambda}
$$

We may write $g=b-a$, where $b$ and $a$ are in $G^{+}$. Then $\tau_{2}(b)>\tau_{2}(a), \tau_{1}(a)>$ $\tau_{1}(b)$ (so $\tau_{1}(a)>0$, and thus $\tau(a)>0$ ), and, most importantly, (*) ensures that $\tau(a)<\tau(b)$. If $0 \leq c \leq b$ and $\tau(c)=\tau(a)$, the latter implies $0=\lambda \tau_{1}(c-a)+$ $(1-\lambda) \tau_{2}(c-a)$. The hypothesis on the intersection forces $\tau_{i}(c-a)=0$, whence $\tau_{1}(c)=\tau_{1}(a)>\tau_{1}(b)$, a contradiction.

Some convex combinations of pure traces are good, e.g., consider $G=\mathbf{Q} \oplus \mathbf{Q}$ with the strict ordering from the obvious inclusion in $\mathbf{R}^{2}$ : a trace given as $\lambda \tau_{1}+(1-\lambda) \tau_{2}$ with $0 \leq \lambda \leq 1$, a weighted average of the two coordinates, is good, if and only if $\lambda$ is rational.

On the other hand, the following trivial result shows that a lot more convex combinations, even with rational choices for $\lambda$, are ruled out.

Lemma 6.2. Let $G=\lim G_{\alpha}$ be a dimension group expressed as a direct limit of ordered abelian groups over a direct set, with maps $\phi_{\alpha}: G_{\alpha} \rightarrow G$. Let $\tau$ be a trace of $G$ such that for all $\alpha, \tau \circ \phi_{\alpha}$ is a good trace of $G_{\alpha}$. Then $\tau$ is good. 
Proof. Pick $a$ and $b$ in $G^{+}$such that $0<\tau(a)<\tau(b)$. There exists (by directedness) $\alpha$ such that $\phi_{\alpha}^{-1}(a) \cap G_{\alpha}^{+}$and $\phi_{\alpha}^{-1}(b) \cap G_{\alpha}^{+}$are both nonempty; choose one from each, say $A$ and $B$ respectively. As $\tau \circ \phi_{\alpha}$ is good, there exists $0 \leq C \leq B$ in $G_{\alpha}^{+}$ such that $\tau \circ \phi_{\alpha}(C)=\tau \circ \phi_{\alpha}(A)$. Then $c:=\phi_{\alpha}(C)$ is the desired element.

If $G$ is a dimension group, then of course it is a direct limit with the $G_{i}$ being simplicial ordered groups ( $\mathbf{Z}^{n(i)}$ with coordinatewise ordering), so if $\tau$ is a trace on $G$, it induces a trace on each of the simplicial ones, necessarily a positive linear combination of the coordinates. Thus if $\tau$ is not good, infinitely many of those traces must themselves be not good (in fact, it is quite easy to find lots of rational combinations of pure traces on simplicial groups that are not good).

\section{GoOdNESS EN MASSE AND REFINABILITY}

Suppose $(G, u)$ is a dimension group with order unit, and $U$ is a set of normalized traces, that is, a subset of $S(G, u)$ (in view of subsequent definitions, we usually assume that $U$ is closed, compact, and convex). We say (the set of traces) $U$ is weakly good if whenever $a$ and $b$ are in $G^{+}$and $\inf _{\tau \in U}(\tau(b)-\tau(a))>0$, then there exists $c \in[0, b]$ such that $\tau(c-a)=0$ for all $\tau$ in $U$. We say $U$ is weakly order unit good if we restrict $b$ to be order units of $G$, and we drop the "weakly" from the definition if $a$ is allowed to vary over all of $G$, not just $G^{+}$, but require $\inf _{\tau \in U} \tau(a)>0$ as well.

Other definitions are possible, and possibly more interesting, e.g., if we only require $\tau(b)-\tau(a)>0$ for all $\tau$ in not necessarily compact $U$, or even $\tau(b) \geq \tau(a)$ for all $\tau$ in $U$. Even in the simple case, these notions are different.

For example, if $U=S(G, u)$, goodness is automatic (a consequence of unperforation; note that none of the individual traces need be good themselves). If $U$ is a singleton, it reverts to the definition of good (or order unit good) that we previously used. The proofs of some results carry over to the general case.

For $Y$ a subset of $G$ (typically of the form $Y=\operatorname{ker} U$ for some subset $U$ of $S(G, u)$ ), we define $Z(Y)=\{\phi \in S(G, u)|\phi| Y \equiv 0\}$. This is a compact convex subset of $S(G, u)$. There is also a generally smaller compact convex set associated to $U, \tilde{U}=L(U) \cap S(G, u)$, where $L(U)$ is the closure of the affine span of $U$ (we shall discuss this construction in more detail later). Then $U$ is good implies $\tilde{U}$ is good, and in this case, $Z(\operatorname{ker} U)=\tilde{U}$ (Lemma 7.8).

The notions of ugly and bad apply to subsets $U$ of $S(G, u)$ as well: $U$ is ugly if the image of ker $U$ is discrete in $\operatorname{Aff} S(G, u)$ and $U$ is good for the group $G \otimes \mathbf{Q} ; U$ is bad if $\operatorname{ker} U=\operatorname{Inf}(G)$, equivalently, $Z(\operatorname{ker} U)=S(G, u)$.

Lemma 7.0 (cf., Lemma 1.1). Let $(G, u)$ be a simple dimension group and let $U$ be a subset of $S(G, u)$.

(a) If for all $a, b \in[0, u]$ such that $0 \ll \widehat{a}|U \ll \widehat{b}| U$ there exists $c \in[0, b]$ such that $\widehat{a}|U=\widehat{c}| U$, then $U$ is good.

Proof. Virtually idential to the proof of Lemma 1.1(a).

Let $U$ be a subset of $S(G, u)$ and define $\operatorname{ker} U=\bigcap_{\tau \in U} \operatorname{ker} \tau$. This is a subgroup of $G$ (frequently zero); define $Z \equiv Z(\operatorname{ker} U)=\{\sigma \in S(G, u) \mid \sigma(\operatorname{ker} U)=0\}$. Then $Z$ is compact, convex, and contains $U$ (it can be bigger than the closed convex hull of $U$ ), and in fact, $Z=S(G, u) \cap \mathcal{L}$ where $\mathcal{L}$ is a closed flat (the intersection of the zero sets of all elements of $\operatorname{ker} U$ ). Note that if for all $\tau$ in $U$ we have 
$\tau(b)-\tau(a) \geq \delta>0$, then the same is true for all $\tau$ in $Z$ (thus goodness of $U$ implies that of $Z$ ). If $Z$ happens to be a face of $S(G, u)$, we will write it as $F$. Let $U^{\vdash}=\{h \in \operatorname{Aff} S(G, u)|h| U=0\}$. This is an order ideal in Aff $S(G, u)$ if the closed convex hull of $U$ is a face of $S(G, u)$, and in that case we write $F^{\perp}$ instead of $U^{\vdash}$.

Let $G=$ Aff $K$ for some Choquet simplex $K$, equipped with the strict ordering. Then we can ask which subsets $U$ of $K$ are good. There is a connection with a classical result on faces: $U$ is good (with respect to $G=\operatorname{Aff} K$ ), equivalently $\tilde{U}$ is good, if and only if for all $f, g \in(\operatorname{Aff} K)^{++}$,

$$
\begin{aligned}
& f|\tilde{U} \ll g| \tilde{U} \quad \text { implies there exists } h \in(\operatorname{Aff} K)^{++} \text {such that } h \ll g \\
& \text { and } h|\tilde{U}=f| \tilde{U} \text {. }
\end{aligned}
$$

A well known result in Choquet theory (e.g., Goo86, 11.25]) asserts that if $F$ is a compact convex subset of $K$, then $F$ is a face if and only if for all $f, g \in(\operatorname{Aff} K)^{+}$,

$$
\begin{aligned}
& f|F \leq g| F \quad \text { implies there exists } h \in(\text { Aff } K)^{+} \text {such that } h \leq f, g \\
& \text { and } h|F=f| F .
\end{aligned}
$$

The second property implies the first (there exists $\epsilon>0$ such that $g^{\prime}=g-\epsilon \mathbf{1} \gg f$ and $f^{\prime}=f-\epsilon \mathbf{1} \gg 0$, and apply the second property to the pair $g^{\prime}, f^{\prime}$, then add $\epsilon \mathbf{1}$ to the resulting $h$ ). The important difference between these two properties is the extra condition that $h \leq f$. It forces the compact convex set $F$ to be a closed face; however, singleton sets $U$ can be good (and these are not faces unless the singleton consists of an extreme point), and we will give constructions of more complicated compact convex sets that are good but not faces. We will also show that $U$ is good for the simple dimension group $(G, u)$ if and only if $\operatorname{ker} U$ has dense image in $\tilde{U}^{\vdash}:=\{h \in \operatorname{Aff} S(G, u)|h| \tilde{U} \equiv 0\}$ and $\tilde{U}$ is good for Aff $S(G, u)$ with the strict ordering.

There is an elementary technicality which can lead to confusion. Let $(H, u)$ be a dimension group. Let $L$ be a subgroup of $H$ with the following properties:

(i) $L \cap H^{+}=\{0\}$;

(ii) for $b$ in $H$ and $n$ a positive integer, if $n b \in L$, then $b \in L$;

(iii) if $b \in H$ and $n b \in L+H^{+}$for some positive integer $n$, then $b \in L+H^{+}$.

In applications, $L=\operatorname{ker} U$ for $U \subset S(G, u)$.

Form the quotient group $H / L$. Impose on it the pre-ordering given by $a+L \geq 0$ if there exists $a^{\prime} \in H^{+}$such that $a-a^{\prime} \in L$. Condition (i) is equivalent to this pre-ordering being a partial ordering; that is, something that is both positive and negative must be zero. Condition (ii) asserts that the quotient group is torsion-free, and condition (iii) is simply a restatement of $H / L$ being unperforated with respect to this ordering (there is some redundance, since unperforation implies torsionfreeness). We are not assuming $L$ is an order ideal; indeed this would defeat the purpose. Typically, $L=\operatorname{ker} U$ where $U \subseteq S(H, u)$. Then (i) and (ii) hold automatically, but (iii) need not, even when $H$ is simple. Appendix B discusses sufficient and necessary conditions for $G / \operatorname{ker} U$ to be unperforated.

If we take the rank 5 critical group $H=\left\langle e_{i} ; \sum_{i=1}^{4} \alpha_{i} e_{i}\right\rangle$ with the strict ordering inherited from $\mathbf{R}^{4}$, with $\left\{1, \alpha_{1}, \alpha_{2}, \alpha_{3}, \alpha_{4}\right\}$ linearly independent over $\mathbf{Q}$, then $H$ is a simple dimension group, with pure traces given by the four coordinate maps, $\tau_{i}$. Let $L=(1,1,-1,-1) \mathbf{Z}$; this is the intersection of the kernels of the four traces $\phi_{1}=\tau_{1}+\tau_{3}, \phi_{2}=\tau_{1}+\tau_{4}, \phi_{3}=\tau_{2}+\tau_{3}$, and $\phi_{4}=\tau_{2}+\tau_{4}$. In particular $H / L$ is 
partially ordered via the quotient ordering. However, we note that $2(1,1,0,0)=$ $(1,1,1,1)+(1,1,-1,-1)$, so $2(1,1,0,0)+L$ is in the positive cone of $H / L$. However, for no integer (positive or negative) is $(1,1,0,0)+m(1,1,-1,-1)$ in $H^{+}$. So $H / L$ is not 2-unperforated. Incidentally, the pure trace space of $H / L$ is $\left\{\phi_{i}\right\}$; since there is an affine relation $\phi_{1}+\phi_{4}=\phi_{2}+\phi_{3}$, the trace space is not a simplex. In this example, $U=\left\{\phi_{i}\right\}$ and $Z(\operatorname{ker} U)$ agrees with $\tilde{U}$ and is the convex hull of $\left\{\phi_{i}\right\}$. We will see more examples of this sort in Appendix B.

However, if (i) holds and both $H$ and $L$ are rational vector spaces, then $H / L$ is torsion-free, and also $H / L$ is unperforated; that is, (iii) holds. The importance of this lies in the following result.

Lemma 7.1. Let $(H, u)$ be an approximately divisible dimension group, and let $L$ be a subgroup of $H$ satisfying (i)-(iii) above. Let $h$ be an element of $H$. If for all traces $\psi$ of $(H, u)$ such that $\psi(L)=0, \psi(h)>0$, then there exists an order unit $v$ of $H$ such that $v-h \in L$.

In particular, this applies if $L$ is of the form $\bigcap_{\tau \in U} \operatorname{ker} \tau$ for some subset $U \subset$ $S(H, u)$ such that $\left(\bigcap_{\tau \in U} \operatorname{ker} \tau\right) \cap H^{+}=\{0\}$ and $H$ is a rational vector space.

Proof. Since $H$ is approximately divisible, its image in Aff $S(H, u)$ is dense, and thus we may impose the strict ordering on $H, H^{++} \cup\{0\}$; that is, take the order units of the original ordering (those with strictly positive image in Aff $S(H, u)$ and make them, together with 0 , the new positive cone. The new ordering on $H$ is also a dimension group ordering (since $S(G, u)$ is a Choquet simplex). The trace space is unchanged, and (i), (ii), (iii) remain in force, since $H^{++} \subseteq H^{+}$.

Impose the quotient ordering on $H / L$ arising from the strict ordering on $H$. Now we examine the normalized traces of $(H / L, u+L)$. If $\phi$ is a trace, then since $\pi: H \rightarrow H / L$ is by construction order preserving, $\phi \circ \pi$ is a trace of $H$, and obviously $\phi \circ \pi(L)=0$. Conversely, if $\psi: H \rightarrow \mathbf{R}$ is a normalized trace of $H$ such that $\phi(L)=0$, then we can construct $\phi \in S(H / L, u+L)$ such that $\phi \circ \pi=\psi$ in the obvious fashion: since $\psi$ kills $L, \psi$ induces a group homomorphism $\phi: H / L \rightarrow \mathbf{R}$ defined as $\phi(a+L)=\psi(a)$; this is obviously well defined, and, moreover, if $a+L \geq 0$ (in $H / L$ ), then there exists $a^{\prime} \geq 0$ (in $H$ ) such that $a-a^{\prime} \geq 0$. Then $\psi(a)=\psi\left(a^{\prime}\right) \geq 0$, and thus $\phi$ is positive, i.e., a trace, and normalization is automatic. It is now routine that the map $S(H / L) \rightarrow S(H, u) \cap L^{\vdash}$ (the latter is the set of traces killing $L)$ given by $\phi \mapsto \phi \circ \pi$ is an affine homeomorphism.

Finally, suppose $\psi(h)>0$ for all $\psi$ in $S(H, u) \cap L^{\vdash}$. Then $\phi(h+L)>0$ for all $\phi \in S(H / L, u+L)$, and since $H / L$ is unperforated, $h+L$ is a positive element of $H / L$, and obviously nonzero. By definition of the quotient ordering, there exists $v$ in $H^{++} \cup\{0\}$ such that $v-h \in L$. Obviously $v \neq 0$ (evaluate at any $\psi$ ), so $v \in H^{++}$; that is, $v$ is an order unit with respect to the original ordering on $H$.

If $L=\bigcap_{\tau \in U} \operatorname{ker} \tau$, then (i) occurs by hypothesis, and (ii) is obvious, and when $H$ is a rational vector space, (iii) is a consequence of (i) and (ii).

From a subset $U$ of $S(G, u)$, we first form $\operatorname{ker} U=\bigcap_{\tau \in U} \operatorname{ker} \tau$, a subgroup of $G$. Reasonably general conditions under which $G / \operatorname{ker} U$ is (or is not) unperforated are given in Appendix B. Next, we form $Z(\operatorname{ker} U)=\{\sigma \in S(G, u) \mid \sigma(\operatorname{ker} U)=0\}$. Then $U \subseteq Z(\operatorname{ker} U)$, and the latter is of the form $S(G, u) \cap W$ where $W$ is a closed flat (the intersection of the zero sets of all the elements of ker $U$ ); it is also a closed convex subset of $S(G, u)$, but as we saw earlier, it need not be a 
simplex itself. Finally, we form a closed convex real vector subspace of Aff $S(G, u)$, $Z(\operatorname{ker} U)^{\vdash}=\{h \in \operatorname{Aff} S(G, u)|h| Z(\operatorname{ker} U) \equiv 0\}$. The representation $g \mapsto \widehat{g}$ sends $\operatorname{ker} U=\operatorname{ker}(Z(\operatorname{ker} U))$ to $Z(\operatorname{ker} U)^{\vdash}$.

A measure $\mu$ on $X$ is refinable if for all clopen $A$, whenever $\mu(A)=\sum \alpha_{i}$ where $\left\{\alpha_{i}\right\}$ is a finite set of elements in the clopen value set $(S(\mu):=\{\mu(B) \mid B$ is clopen $\})$, there exist pairwise disjoint clopen sets $V_{i}$ such that $\mu\left(V_{i}\right)=\alpha_{i}$ and $A=\bigcup V_{i}$. Good measures are refinable, but the converse fails. Refinability is related to goodness en masse, as observed in Proposition 7.6 and Lemma 7.8.

This definition translates directly to dimension groups. Let $(G, u)$ be a dimension group with order unit; a normalized trace $\tau$ is refinable with respect to $u$ if for all $b, a_{1}^{\prime}, \ldots, a_{n}^{\prime}$ in $[0, u]$ such that $\tau(b)=\sum \tau\left(a_{i}^{\prime}\right)$, there exist $a_{i} \in G^{+}$such that $\tau\left(a_{i}\right)=\tau\left(a_{i}^{\prime}\right)$ and $b=\sum a_{i}$. If $\tau$ is refinable with respect to all order units (that is, the $b$ and $a_{i}^{\prime}$ are allowed to be arbitrary elements of $\left.G^{+}\right)$, then we simply say $\tau$ is refinable. It probably is the case that refinability with respect to a single order unit implies refinability.

Analogous to goodness, we can define refinability for a set of traces. For a dimension group with order unit $(G, u)$, and $U \subseteq S(G, u)$, we say that $U$ is refinable (with respect to $u$ ) if whenever $b, a_{i}^{\prime} \in[0, u]$ and $\tau(b)=\sum \tau\left(a_{i}^{\prime}\right)$ for all $\tau$ in $U$, there exist $a_{i} \in G^{+} \operatorname{such}$ that $b=\sum a_{i}$. If we let $L=\operatorname{ker} U=\bigcap_{\sigma \in U} \operatorname{ker} \sigma$, then the hypothesis is obviously $b-\sum a_{i}^{\prime} \in L$, and this is equivalent to $\widehat{b} \mid U=$ $\sum \widehat{a}_{i}^{\prime} \mid U$, and this in turn is equivalent to $\widehat{b}\left|Z=\sum \widehat{a}_{i}^{\prime}\right| Z$ where $Z=Z(\operatorname{ker} U)=$ $\{\sigma \in S(G, u) \mid \sigma(\operatorname{ker} U)=0\}$. Note that refinability of $\tau$ is equivalent to refinability of $Z(\operatorname{ker} \tau)$. The latter is of the form $S(G, u) \cap \mathcal{L}$ where $\mathcal{L}$ is a closed flat. We will see below that refinability of $\tau$ forces $Z(\operatorname{ker} \tau)$ to be a simplex, a fairly severe restriction.

If $\operatorname{ker} U=\{0\}$, then $Z(\operatorname{ker} U)=S(G, u)$, which of course is good; therefore, $U$ is refinable. This generalizes the trivial fact that a bad trace ( $\operatorname{ker} \tau=\{0\})$ is refinable; see Lemma 7.3 below.

An element $b$ of $G^{+}$is called refinable with respect to $\tau$ if whenever $\tau(b)=\sum \tau\left(a_{i}^{\prime}\right)$ with $a_{i}^{\prime} \in G^{+}$, there exist $a_{i}$ in $G^{+}$such that $b=\sum a_{i}$. Obviously $\tau$ is refinable if and only if every $b$ in $G^{+}$is refinable with respect to $\tau$. We say $\tau$ is weakly refinable if there exists a generating set $H$ of the positive cone such that every member of $H$ is refinable with respect to $\tau$. Unlike the corresponding concepts for goodness, it is not clear that weak refinability implies refinability. First, however, we have a skirmish of the definitions.

Lemma 7.2. Suppose $(G, u)$ is a dimension group, $\tau$ is a trace that is group-like with respect to $u$, and $b$ is an element of $[0, u]$ that is refinable with respect to $\tau$. Then $b$ is $\tau$-good.

Proof. Recall that $\tau$ is group-like with respect to $u$ asserts that $\tau([0, u])=\tau(G) \cap$ $[0, \tau(u)]$. Suppose $a$ is an element of $G$ such that $0<\tau(a), \tau(b-a)<\tau(b) \leq \tau(u)$. Since $u$ is $\tau$-good, there exist $c, c^{\prime} \in[0, u]$ such that $\tau(c)=\tau(a)$ and $\tau\left(c^{\prime}\right)=\tau(b-a)$. Thus $\tau(b)=\tau(c)+\tau\left(c^{\prime}\right)$, and now we can apply refinability of $b$ to obtain $d$ and $d^{\prime}$ in $G^{+}$such that $b=d+d^{\prime}$, and $d-c, d^{\prime}-c^{\prime} \in \operatorname{ker} \tau$. In particular, $d$ belongs to $[0, b]$ and $\tau(d)=\tau(a)$, so $b$ is $\tau$-good.

Not only are good traces refinable, so are the bad ones (by a completely trivial argument)! 
Lemma 7.3. Let $(G, u)$ be a dimension group with order unit, and let $\tau$ be a trace. Any of the following conditions is sufficient for $\tau$ to be refinable:

(a) $\tau$ is good.

(b) $\operatorname{ker} \tau=\operatorname{Inf}(G)$ and $G$ is simple.

(c) $\operatorname{ker} \tau=\{0\}$.

Proof. (a) Given $b, a_{i}^{\prime} \in[0, u]$, we may assume that $\tau(b)>0$ (or else just replace $a_{1}^{\prime}$ by $b$ and the rest by 0 ) and relabel so that $\tau(b)>\tau\left(a_{1}^{\prime}\right)$ (if $\tau(b)=\tau\left(a_{1}^{\prime}\right)$, set $a_{1}=b$ and the rest zero); by goodness, there exists $a_{1} \in[0, b]$ such that $\tau\left(a_{1}\right)=\tau\left(a_{1}^{\prime}\right)$, and apply the same procedure to $\tau\left(b-a_{1}\right)=\sum_{i \geq 2} \tau\left(a_{i}^{\prime}\right)$. The process stops when (after relabelling) $\tau\left(\sum_{i \geq l} a_{i}^{\prime}\right)=0$, in which case we set $a_{l}=b-\sum_{i \leq l-1} a_{i}^{\prime}$ and $a_{l+j}=0$ for $j>0$.

(b) Since $\tau(b)=\sum \tau\left(a_{i}^{\prime}\right)$, we have $b-\sum a_{i}^{\prime}:=k$ is an infinitesimal; replace one of the nonzero $a_{j}^{\prime}$ by $a_{j}=a_{j}+k$, so that $a_{j}$ is still an order unit, and thus positive, and set all the other $a_{i}^{\prime}=a_{i}$.

(c) Obviously $\tau(b)=\sum \tau\left(a_{i}^{\prime}\right)$ implies $b=\sum a_{i}^{\prime}$.

As an elementary exercise, if $G$ is a simple dimension group with exactly two pure traces and $\operatorname{Inf}(G)=0$, then a refinable trace is either good or bad. This fails with three pure traces (Example 11, $G^{\prime}$ ).

Corollary 7.4. Let $(G, u)$ be a dimension group with order unit, and let $\tau$ be a trace of $G$. The following are equivalent:

(a) $\tau$ is good,

(b) $\tau$ is both group-like and refinable with respect to $u$,

(c) $\tau$ is both group-like and weakly refinable with respect to $u$.

Proof. (a) $\Longrightarrow$ (b) is an immediate consequence of Lemma 7.2 and the definitions, and $(\mathrm{b}) \Longrightarrow(\mathrm{c})$ is trivial.

(c) $\Longrightarrow$ (a). Let $H$ be a generating set for $G^{+}$consisting of refinable elements. We observe that $H(u):=H \cap[0, u]$ is also a generating set: if $g \in G^{+}$and $g \leq n u$, there exist $u_{i} \in[0, u]$ such that $g=\sum u_{i}$. Since each $u_{i}$ is represented as a sum of elements of $H$, each of which must lie in $[0, u], H(u)$ is a generating set. By Lemma 7.2 , every element of $H(u)$ is $\tau$-good, and so by Lemma 1.3, all elements of $G^{+}$are $\tau$-good, and thus $\tau$ is good.

The following is immediate from the definitions.

Lemma 7.5. Let $(G, u)$ be a dimension group, and suppose that $L$ is a subgroup satisfying (i) and (ii), so that $G / L$ with the quotient ordering is a torsion-free partially ordered abelian group; let $\pi: G \rightarrow G / L$ denote the quotient map. Set $Z=\{\phi \in S(G, u) \mid \phi(L)=0\}$. The map $S(G / L, u+L) \rightarrow Z$ given by $\psi \mapsto \psi \circ \pi$ is an affine homeomorphism.

The following does not yield the expected result in which $G / \operatorname{ker} U$ is a dimension group if $U$ is refinable; this would require $G / \operatorname{ker} U$ to be unperforated, which is not clear at the moment. It is, however, simple and torsion-free. Simple torsionfree interpolation groups which are not unperforated exist; the first example was constructed by John Lawrence $[\mathrm{L}]$.

However, if $U$ is good (with respect to $G$ ) and $G$ is simple, then we will show that $G / \operatorname{ker} U$ is unperforated, en route to showing it is a simple dimension group. Let $J$ be a torsion-free partially ordered abelian group (which will be set to $G / \operatorname{ker} U$ ), not 
assumed to be unperforated, and let $a$ be an element of $J$. Define a (possibly empty) subset of $\mathbf{N}$ (the set of strictly positive integers), $P(a)=\left\{n \in \mathbf{N} \mid n a \in J^{+}\right\}$. We note that $P(a)$ is closed under addition, so if $P(a)$ contains a subset whose greatest common divisor is 1 , then $P(a)$ is cofinite. In general, $P(a)$ is either empty or of the form $k \mathbf{N} \backslash T$ for some positive integer $k$ and a finite set $T$.

If $J$ is simple, then $P(a)$ is either empty or cofinite. For nonzero $a$, if $P(a)$ is not empty, choose $n \in \mathbf{N}$ such that $n a \in J^{+}$; since $J$ is torsion-free, $n a$ is nonzero, and hence (being in $J^{+}$) is an order unit. Thus there exists a positive integer $N$ such that $a \leq N n a$. This implies $N n-1 \in P(a)$, and $\operatorname{since} \operatorname{gcd}(n, N n-1)=1$, $P(a)$ is cofinite.

Proposition 7.6. Suppose $(G, u)$ is a simple dimension group. Let $U$ be a collection of traces, and form $Z(\operatorname{ker} U)=\{\sigma \in S(G, u) \mid \sigma(\operatorname{ker} U)=0\}$.

(a) If $U$ or $Z(\operatorname{ker} U)$ is refinable, then the quotient group $G / \operatorname{ker} U$ satisfies Riesz interpolation, and thus $Z(\operatorname{ker} U)$ is a simplex.

(b) If $U$ is good, then $G / \operatorname{ker} U$ is unperforated.

(c) If $U$ is good, then $U$ is refinable.

(d) If $U$ is good, then $G / \operatorname{ker} U$ is a simple dimension group.

(e) If $Z(\operatorname{ker} U)$ is refinable and $G / \operatorname{ker} U$ is unperforated, then $\operatorname{ker} U$ has dense image in $Z(\operatorname{ker} U)^{\vdash}$.

(f) If $Z(\operatorname{ker} U)$ is refinable and $G / \operatorname{ker} U$ is unperforated, then $Z(\operatorname{ker} U)$ is good.

Proof. (a) It suffices to show $G / \operatorname{ker} U$ satisfies interpolation. By [Goo86, 2.1(c)], sufficient is that if $a_{i}, b_{i}$ are positive elements of $G / \operatorname{ker} U$ such that $b_{1}+b_{2}=a_{1}+a_{2}$, then we may find $a_{i j} \in(G / \operatorname{ker} U)^{+}$such that $b_{i}=\sum_{j} a_{i j}$ and $a_{j}=\sum_{i} a_{i j}$. If any of $\left\{a_{i}, b_{j}\right\}$ is zero, there is nothing to do. Since $a_{i}, b_{j}$ are in $(G / \operatorname{ker} U)^{+}$, by definition of the quotient ordering, there exist $A_{i}, B_{j} \in G^{++}$such that $a_{i}=A_{i}+\operatorname{ker} U$, $b_{j}=B_{j}+\operatorname{ker} U$. Then $B_{1}+B_{2}=A_{1}+A_{2}-k$ for some $k \in \operatorname{ker} U$. By refinability applied to the $A_{i}$, there exist positive elements $A_{i}^{\prime}$ such that $A_{i}-A_{i}^{\prime} \in \operatorname{ker} U$ and $B_{1}+B_{2}=A_{1}^{\prime}+A_{2}^{\prime}$. Now interpolation applies in $G$, yielding $A_{i j}$ in $G^{+}$satisfying the usual equations, and their images $a_{i j}=A_{i j}+\operatorname{ker} U$ satisfy the corresponding equations, yielding the Riesz interpolation property. By Lemma 7.5, the trace space of $G / \operatorname{ker} U$ is affinely homemorphic to $Z$ (via $\psi \mapsto \psi \circ \pi$ ), and it follows that $Z$ is a simplex.

(b) Let $a$ be a nonzero element of $J=G / \operatorname{ker} U$ with nonempty $P(a)$. Set $L=\operatorname{ker} U$. Suppose $m, n \in P(a)$ with $m>n$. We show that $m-n \in P(a)$. Since $m a$ and $n a$ are in the positive cone of $G / L$, from the definition of the quotient ordering, there exist $A, B$ in $G^{+}$such that $B+L=m a$ and $A+L=n a$. Since $G$ is simple, $\tau(B)$ is bounded below away from zero as $\tau$ varies over $S(G, u)$, and thus if $\tilde{\tau} \in S(G / L, u+L)$ corresponds to $\tau \in Z, \tilde{\tau}(m a)$ is bounded below away from zero. Hence $\tau(B)=\tilde{\tau}(m a)=m \tilde{\tau}(a)>n \tilde{\tau}(a)=\tau(A)$ and the difference is bounded below away from zero. Thus in $G$, we have $\widehat{B}|Z-\widehat{A}| Z$ is bounded below away from zero, that is, $\tau(B-A)$ is bounded below as $\tau$ varies over $U$ or $Z$. By goodness of $U$, there exists $A^{\prime} \in[0, B]$ such that $A-A^{\prime} \in \operatorname{ker} Z$. Thus $B-A^{\prime} \in G^{+}$, so its image in $G / L,(m-n) a$, belongs to $(G / L)^{+}$, so $m-n \in P(a)$. This forces $P(a)=k \mathbf{N}$ for some nonnegative integer $k$ (if $P(a)$ is nonempty, let $k$ be its smallest element, and use the division algorithm). Since $J$ is simple, $P(a)$ is either cofinite or empty, hence $k=1$, and thus $J$ is unperforated. 
(c) Suppose $a_{i}^{\prime}, b \in G^{+}$and $b-\sum_{i=1}^{n} a_{i}^{\prime}=k \in \operatorname{ker} U$. Assuming $a_{1} \neq 0$, we have $a_{1}^{\prime}$ is thus an order unit; hence there exists $\delta>0$ such that $\widehat{a}_{1} \gg \delta \mathbf{1}$. Hence $\widehat{b}\left|U-\widehat{a}_{n}\right| U \gg \delta \mathbf{1}$, whence $b-a_{n}^{\prime}+\operatorname{ker} U$ is an order unit of $G / \operatorname{ker} U$. We may thus lift $b-a_{n}^{\prime}+\operatorname{ker} U$ to an order unit $c^{\prime}$ of $G^{+}$; since $b-c^{\prime}+\operatorname{ker} U=a_{n}^{\prime}+\operatorname{ker} U$, goodness of $U$ applies. There thus exists $c \in[0, b]$ such that $c^{\prime}-c \in \operatorname{ker} U$. Now $b-c=\sum_{1}^{n-1} a_{i}+\left(k+c-a_{n}\right)$. The parenthesized term is in $\operatorname{ker} U$, and induction can now be applied.

(d) By (c) and (a), $G / \operatorname{ker} U$ satisfies interpolation; by (b), it is unperforated. Simplicity is automatic.

(e) Select $h$ in $Z^{\vdash}=\{f \in \operatorname{Aff} K|f| Z \equiv 0\}$. By approximate divisibility and consequent density of $G$ in Aff $K$, given $\epsilon>0$, there exists $g \in G$ such that $\|\widehat{g}-h\|<$ $\epsilon$. In particular, for all $\tau \in Z,|\tau(g)|<\epsilon$. By approximate divisibility again, there exists a (small) order unit, $e$, such that $\epsilon \mathbf{1}<\widehat{e}<2 \epsilon \mathbf{1}$. Hence $\tau(g+e)>0$ for all $\tau$ in $Z$, and $\|\widehat{g}+\widehat{e}-h\|<3 \epsilon$. Set $g_{1}=g+e$.

Since $\widehat{g}_{1} \mid Z \gg 0$, the element $g_{1}+\operatorname{ker} U$ is an order unit of the unperforated group $G / \operatorname{ker} U$ (ker $U$ automatically satisfies (i) and (ii), and by hypothesis (iii)); hence there exists an order unit $v$ of $G$ such that $\widehat{v}\left|Z=\widehat{g}_{1}\right| Z$. Let $f$ be a (small) order unit of $G$ such that $3 \epsilon<\widehat{f}<4 \epsilon$. Since $\widehat{g}_{1} \mid Z<3 \epsilon$, it follows that $(\widehat{f}-\widehat{v}) \mid Z$ is an order unit of $G / \operatorname{ker} U$. There thus exists an order unit $w$ of $G$ such that $(\widehat{f}-\widehat{v}) \mid Z=\widehat{w} Z$. Thus $(\widehat{f}-\widehat{w}-\widehat{v}) \mid Z \equiv 0$; hence there exists $k$ in $\operatorname{ker} U$ such that $f=w+v+k$. Since $f, w$, and $v$ are all order units, and hence are positive, we can apply refinability. There thus exist positive $w^{\prime}$ and $v^{\prime}$ such that $w^{\prime}-w \in \operatorname{ker} U, v^{\prime}-v \in \operatorname{ker} U$ and $f=w^{\prime}+v^{\prime}$. Thus $v^{\prime} \in[0, f]$, so $\left\|\widehat{v}^{\prime}\right\|<4 \epsilon$.

Now consider $g^{\prime}:=g_{1}-v^{\prime}$. Restricted to $Z$, its value is zero (since $v^{\prime}|Z=v| Z=$ $\left.g_{1} \mid Z\right)$, so $g^{\prime} \in \operatorname{ker} U$. On the other hand $\left\|\widehat{g}^{\prime}-h\right\| \leq\left\|\widehat{g}_{1}-h\right\|+\left\|v^{\prime}\right\|<3 \epsilon+4 \epsilon=7 \epsilon$. So ker $U$ has dense image in $Z(\operatorname{ker} U)^{\vdash}$.

(f) The quotient being unperforated implies that order units lift (Lemma 7.1). Suppose $a$ and $b$ are nonzero elements of $G^{+}$(therefore are order units) such that $\tau(b)-\tau(a)>0$ for all $\tau$ in $Z$. Since the set of traces of the quotient induce the elements of $Z, b-a+\operatorname{ker} U$ is an order unit of $G / \operatorname{ker} U$. Since order units lift, there exists an order unit $c$ in $G$ such that $c-b+a \in \operatorname{ker} U$. Hence $\tau(b)=\tau(a)+\tau(c)$ for all $\tau$ in $Z$. By refinability of $Z$, there exist $a^{\prime}, c^{\prime} \geq 0$ such that $b=a^{\prime}+c^{\prime}$; thus $a^{\prime} \in[0, b]$ and $a^{\prime}-a \in \operatorname{ker} U$.

It follows that for the rank 5 critical group given just before Lemma 7.1, the set $U$ consisting of the four extreme traces of $G / L$ (or its convex hull, a square in $\mathbf{R}^{4}$ ) is not a good subset of $S(G \otimes \mathbf{Q}, u$ ) (for any choice of order unit) even after tensoring $G$ with the rationals. On the other hand, all the individual traces are good with respect to $G \otimes \mathbf{Q}$.

A particular consequence of (b) is that if $Z(\operatorname{ker} U)$ is good, then $U$ is refinable (since $U$ is refinable if and only if $Z(\operatorname{ker} U)$ is refinable). The converse is almost true; it may be that refinability implies the quotient is unperforated. If $G$ is divisible, then $G / \operatorname{ker} U$ is automatically unperforated, and in this case, refinability of $U$ is equivalent to goodness of $Z(\operatorname{ker} U)$.

The difference between $U$ being good and $U$ being refinable should be clarified. We have seen that $U$ is refinable if and only if $Z(\operatorname{ker} U)$ is refinable, and the latter is of the form $K \cap \mathcal{L}$ where $K$ is the trace space, and $\mathcal{L}$ is a closed flat obtained as an intersection of zero sets of $\widehat{g}$ where $g \in U$, meaning $\mathcal{L}=\bigcap_{g \in \operatorname{ker} U} \widehat{g}^{-1}(0)$. There is a similar characterization for good $U$, involving a (generally) smaller flat. 
For a set $U \subset K$, define $L(U)$ to be the smallest closed flat containing $U$; that is, $L(U)$ is the closure of the set $\left\{\sum \alpha_{i} u_{i} \mid \alpha_{i} \in \mathbf{R} ; \sum \alpha_{i}=1 ; u_{i} \in U\right\}$. Define $\widetilde{U}=K \cap L(U)$. If $U$ is a singleton, then $L(U)=\widetilde{U}=U$, and in general, $\widetilde{U}$ is a compact convex set contained in $Z(\operatorname{ker} U)$. Alternatively, we could define $\widetilde{U}$ as $K \cap\left(\bigcap_{\{h \in \text { Aff } K|h| U \equiv 0\}} h^{-1}(0)\right)$. Both are of the form $K \cap \mathcal{L}$ where $\mathcal{L}$ is a closed flat.

There is a property related to goodness, which, however, does not seem to lend itself to any particular use. We say that a subset $U$ of a dimension group $(G, u)$ with order unit is poob (this is what handwritten good approximately looks like when viewed upside down) if for all $a$ and $b$ in $G^{+}$such that $\tau(b)-\tau(a) \geq \delta>0$ for some $\delta$ and all $\tau$ in $U$, there exists $b^{\prime}$ in $G^{+}$such that $\tau(b)=\tau\left(b^{\prime}\right)$ and $a \leq b^{\prime}$.

Proposition 7.7. Suppose that $(G, u)$ is simple and not cyclic, and $U$ is a subset of $S(G, u)$. If $\operatorname{ker} U$ has dense image in $Z(\operatorname{ker} U)^{\vdash}$, then $U$ and $Z(\operatorname{ker} U)$ are poob. In particular, if $U$ is good, then it is poob.

Remark. But not conversely! By Proposition 7.6(a), if $U$ is good, then $Z(\operatorname{ker} U)$ is a simplex, but we have already seen a finite-dimensional example for which $\operatorname{ker} U$ has dense image, but $Z(\operatorname{ker} U)$ is not a simplex.

Proof. Suppose $a$ and $b$ are in the positive cone of $G$ and $\tau(b)-\tau(a)>\delta>0$ for all $\tau \in U$ and some $\delta>0$. By definition, the same inequality holds for all $\tau$ in $Z(\operatorname{ker} U)$. Let $H=\operatorname{Aff} S(G, u)$ and $L=Z(\operatorname{ker} U)^{\perp}$. Applying Lemma 7.1 to $\widehat{b}-\widehat{a}$ (restricted to $Z(\operatorname{ker} U)$, this element is strictly positive), there exists $f \in \operatorname{Aff} S(G, u)$ such that $f \gg 0$ and $f|Z(U)=(\widehat{b}-\widehat{a})| Z(U)$; that is, $e:=f-(\widehat{b}-\widehat{a})$ is in $Z(U)^{\perp}$.

Given $\epsilon>0$, there exists (by the density hypothesis) $j \in \operatorname{ker} U$ such that $\|\widehat{j}-e\|<$ $\epsilon$, so that ||$\widehat{j}-\widehat{a}+\widehat{b}-\widehat{f} \mid<\epsilon$. Hence if $\epsilon$ is chosen less than $\inf _{\sigma \in S(G, u)} f(\sigma)$ (this is of course strictly positive, since $f$ is a continuous strictly positive function on a compact space), then $j-a+b$ is an order unit of $G$. In particular, $b+j \gg a$, so the former is positive, and we can set $b^{\prime}=b+j$.

If $U$ is good, then Lemma 7.8(a) and Proposition 7.6(a), (c) apply to yield density.

The following is almost immediate from the definitions.

Lemma 7.8. Let $(G, u)$ be an approximately divisible dimension group and $U$ be a subset of $S(G, u)$.

(a) If $U$ is a good subset of $S(G, u)$, then $\widetilde{U}$ is a good subset of $S(G, u)$, and in that case, $Z(\operatorname{ker} U)=\widetilde{U}$ and is a simplex.

(b) $U$ is a refinable subset of $S(G, u)$ if and only if $Z(\operatorname{ker} U)$ is a refinable subset of $S(G, u)$.

Proof. The only assertion that is not tautological is that if $U$ is good, then $Z(\operatorname{ker} U)$ $=\widetilde{U}$. Suppose $h \in \operatorname{Aff} K$ vanishes identically on $U$; we show $h$ is approximable by the images of elements of $\operatorname{ker} U$. By approximate divisibility, given $\epsilon>0$, there exists $g_{1} \in G$ such that $\left\|h-\widehat{g}_{1}\right\|<\epsilon$. Then $\left|\tau\left(g_{1}\right)\right|<\epsilon$ for all $\tau \in U$. There exist small order units $v$ and $b$ of $G$ such that $\epsilon \mathbf{1}<\widehat{v}<2 \epsilon \mathbf{1}$ and $4 \epsilon \mathbf{1}<\widehat{b}<5 \epsilon \mathbf{1}$ (again, by density of $\widehat{g}$ in Aff $K$, a consequence of approximate divisibility). Then $\widehat{v}+\widehat{g}_{1}$ is strictly positive on $U$. By goodness applied to $b$ and $v+g_{1}$, there exists $c \in[0, b]$ such that $\widehat{c}|U=\widehat{v}| U+\widehat{g}_{1} \mid U$, that is, $\tau(c)=\tau\left(v+g_{1}\right)$ for all $\tau \in U$. Since 
$0 \leq c \leq b$, it follows that $\|c\| \leq\|b\|<5 \epsilon$. Set $g=v+g_{1}-c$; this belongs to $\operatorname{ker} U$ and $\|\widehat{c}-h\| \leq\left\|\widehat{g}_{1}-h\right\|+\|\widehat{v}\|+\|\widehat{c}\|<8 \epsilon$.

Thus the image of $\operatorname{ker} U$ is dense in $U^{\vdash}$, and the equality $Z(\operatorname{ker} U)=\widetilde{U}$ follows.

While Lawrence's example of a simple torsion-free interpolation group is complicated, we can give an easy example of a simple torsion-free partially ordered group with exactly two pure traces (thus the trace space is a simplex) for which unperforation fails (unfortunately, it is not clear whether interpolation holds). Here $G$ is a simple dimension group with three traces, $U$ is a subset of $S(G, u), Z(U)$ is a line segment and $G / \operatorname{ker} U$ is not unperforated.

Consider the rank 4 critical group of the form $G=\left\langle e_{1}, e_{2}, e_{3} ; \sum \alpha_{i} e_{i}\right\rangle$ in $\mathbf{R}^{3}$ with strict ordering inherited from $\mathbf{R}^{3}$; the pure traces are the evaluations at the coordinates, $\tau_{i}$. Let $U=\left\{\left(2 \tau_{1}+3 \tau_{2}\right) / 5,\left(3 \tau_{1}+5 \tau_{3}\right) / 8\right\}$ (the denominators are normalizations at the order unit $(1,1,1)$; they can be dispensed with). Then $Z(\operatorname{ker} U)$ is a line segment running from a point on the edge joining $\tau_{1}$ to $\tau_{2}$ to a point on the edge joining $\tau_{1}$ to $\tau_{3}$, therefore a simplex. It is easy to calculate $\operatorname{ker} U=k \mathbf{Z}$ where $k=(15,-10,-9)$.

Now consider the element $g=(2,-1,-1)$. We note that $8 g-k=(1,2,1) \gg$ $(0,0,0)$, so that $8(g+\operatorname{ker} U) \in(G / \operatorname{ker} U)^{+}$. However, there is no integer $n$ such that all three coordinates of $(2,-1,-1)+n(15,-10,-9)$ are positive: the first coordinate would require $n \geq 0$, but the second requires $n<0$. So $G / \operatorname{ker} U$ is not 8-unperforated, even though $Z$ is a simplex. In addition, $17 g-2 k$ belongs to $G^{+}$, and this means that there exists $m$ such that for all $t \geq m, t(g+\operatorname{ker} U) \in(G / L)^{+}$ (we can take $m=112=(8-1) \times(17-1)$, probably much less). A similar but easier example is discussed in Appendix B.

What is missing from the litany of implications in Proposition 7.6 is that if $Z=Z(\operatorname{ker} U)$ has dense image in $Z(\operatorname{ker} U)^{\vdash}$ and $Z(\operatorname{ker} U)$ is a simplex, then $Z$ is good for $G$. This is false as we will see in Lemma 7.11 , but it can be reduced to a problem concerning Aff $K$ ( $K$ a Choquet simplex). If $Z$ happens to be a face, then it is good, as will be a consequence of an easy computation, and as we saw is a consequence of a liftability statement about faces in Choquet simplices. There are however lots of choices of $Z$ which are not faces, which we will show to be good.

Another question arising from this result is whether refinability of $U$ implies $G / \operatorname{ker} U$ is unperforated-which would imply that $U$ is refinable if and only if $Z(\operatorname{ker} U)$ is good.

The sets $Z(\operatorname{ker} U)$ create a Zariski-like topology on $S(G, u)$; this topology depends on $G$, not just the simplex $S(G, u)$ - the closed sets come from intersections of kernels of traces; that is, $Z(\operatorname{ker} U)$ is of the form $S(G, u) \cap \mathcal{L}$, where $\mathcal{L}$ is the closed flat obtained as the intersection of zero sets of elements of $\operatorname{ker} U$. Good closed sets are of the form $Z(\operatorname{ker} U)$, where the latter is a simplex and $\operatorname{ker} U$ is dense in $\tilde{U}^{\vdash}$ (not $Z^{\vdash}$ ), but these conditions are not sufficient. In case $G=$ Aff $K$ itself, this topology extends the facial topology (defined on $\partial_{e} K$ ).

Fix $Z \subseteq K$ (both simplices); $g \in \operatorname{ker} Z$ is equivalent to $\widehat{g} \mid Z \equiv 0$. Define $R(G, Z)=\left\{(a, b) \in G^{++} \times G^{++} \mid\right.$there exists $j \in \operatorname{ker} Z$ such that $\left.0 \ll \widehat{a}-\widehat{j} \ll \widehat{b}\right\}$.

Lemma 7.9. Let $(G, u)$ be a simple dimension group. Then $R(G, Z)$ is an open subset of $G^{++} \times G^{++}$with respect to the supremum pseudo-norm; explicitly, for 
all $(a, b) \in R(G, Z)$, there exists $\epsilon>0$ such that if $\|\widehat{a}-h\|,\|\widehat{b}-k\|<\epsilon$, then $(h, k) \in R(\operatorname{Aff} S(G, u), Z$ ) (as usual, $K=S(G, u)$; in addition, in this case, $\operatorname{ker} Z$ is replaced by $\left.Z^{\vdash}\right)$.

Proof. The condition for membership in $R$ is equivalent to $\widehat{a} \gg \widehat{j} \gg \widehat{a}-\widehat{b}$ (for some $j$ in ker $Z$ ). Hence if we pick $\epsilon<\min \left\{\inf _{\sigma \in K} \sigma(a-j), \inf _{\sigma \in K} \sigma(j-a+b)\right\} / 2$, we can use the image of the same $j, \widehat{j}$, for $(h, k)$. A particular consequence is that if $Z(\operatorname{ker} U)$ is good with respect to $G$, then it is also good with respect to Aff $S(G, u)$. The same argument also shows that if $\left(a^{\prime}, b^{\prime}\right) \in G^{++} \times G^{++}$, and $\left\|\widehat{a}-\widehat{a}^{\prime}\right\|$ and $\left\|\widehat{b}-\widehat{b}^{\prime}\right\|$ are sufficiently small, then $\left(a^{\prime}, b^{\prime}\right) \in R(G, Z)$.

Lemma 7.10. Let $(G, u)$ be a simple dimension group, let $U \subseteq S(G, u)$, and let $Z \equiv Z(\operatorname{ker} U)$ denote $\{\sigma \in S(G, u) \mid \sigma(\operatorname{ker} U)=0\}$. The following are equivalent:

(i) $Z$ is good as a family of traces on $\operatorname{Aff} S(G, u)$ (with respect to the strict ordering) and $\operatorname{ker} U$ has dense range in $Z^{\vdash}=\{f \in \operatorname{Aff} S(G, u)|f| Z \equiv 0\}$.

(ii) $Z$ is good as a family of traces on $G$.

Proof. (i) $\Longrightarrow$ (ii) We may replace $U$ by $Z$, since $\widehat{c} \mid Z$ is uniquely determined by $\widehat{c} \mid U$ for all $c$ in $G$. If $a, b \in G^{+}, 0 \ll \widehat{a}|Z \ll \widehat{b}| Z$, then from $Z$ being good as a set of traces on Aff $S(G, u)$, there exists $h$ such that $0 \ll h \ll \widehat{b}$ and $h|Z=\widehat{a}| Z$. Hence $R$ (Aff $S(G, u), Z)$ contains $(\widehat{a}, \widehat{b})$. There thus exists $k \in Z^{\vdash}$ such that $\widehat{a} \gg k \gg \widehat{a}-\widehat{b}$. For $\epsilon<\min \left\{\min _{\sigma \in S(G, u)}(\widehat{a}-k)(\sigma), \min _{\sigma \in S(G, u)}(k-\widehat{a}+\widehat{b})(\sigma)\right\}$, there exists $j$ in $\operatorname{ker} Z=\operatorname{ker} U \subset G$ such that $\|\widehat{j}-k\|<\epsilon$. Then $\widehat{a} \gg \widehat{j} \gg \widehat{a}-\widehat{b}$, and since $G$ is unperforated, $j \in R(G, U)$, hence the latter is nonempty. Thus $U$ is good.

(ii) $\Longrightarrow$ (i) Density follows from Proposition 7.6(a), (c). To show $Z$ is good relative to Aff $S(G, u)$, suppose $f, h \gg 0$ are elements of $\operatorname{Aff} S(G, u)$ such that $h|Z \ll f| Z$. By density of the image of $G$, given $\epsilon$, there exists $a \in G$ such that $h \gg \widehat{a}$ and $h-\widehat{a} \ll \epsilon \mathbf{1}$, and by making $\epsilon$ sufficiently small, we can ensure that $\widehat{a} \gg 0$, so that $a$ is an order unit. Next, we may approximate $f$ by $\widehat{b}$ with $b \in G$ such that $3 \epsilon \mathbf{1} \gg f-\widehat{b} \gg h-\widehat{a}$. In particular, if $\epsilon$ is sufficiently small, it will follow that $\widehat{b} \gg \widehat{a}$. By goodness of $U$ (or of $Z$ relative to $G$ ), there exists $j$ in $\operatorname{ker} U$ such that $\widehat{a} \gg \widehat{j} \gg \widehat{a}-\widehat{b}$. However, $h \gg \widehat{a}$ and $\widehat{a}-\widehat{b} \gg h-f$, yielding $h \gg \widehat{j} \gg h-f$, and thus $f \gg h-j \gg 0$.

By Lemma 7.10 and Proposition 7.6, deciding whether $Z \equiv Z(\operatorname{ker} U)$ is good with respect to $G$ boils down (assuming $\operatorname{ker} U$ has dense image in $Z^{\vdash}$ ) to whether $Z$ is good for the huge group Aff $S(G, u)$. For example, this is the case if $Z=\{\tau\}$ (a singleton) for any $\tau \in K$ (that is, any singleton is a good set of traces for Aff $K$ ). If $Z$ happens to be a face, then $Z$ being good is simply a consequence of the lifting property discussed near the beginning of this section; all that is really necessary is the interpolation property of the usual ordering.

An example wherein a face arises out of a simple dimension group occurs in our standard critical example, $G=\left\{e_{i}, \sum \alpha_{j} e_{j}\right\}$. Then ker $\tau_{1}$ is spanned by elements whose images in Aff $S(G, u)=\mathbf{R}^{n}$ are nonnegative, $\left\{e_{i}\right\}_{i \neq 1}$, and $Z\left(\operatorname{ker} \tau_{1}\right)$ is the face with vertices $\left\{\tau_{2}, \ldots, \tau_{n}\right\}$. Another rather odd example is the line segment from a vertex to a point in the relative interior of a facet. This is neither a face nor a singleton; however, not many other types of $Z$ can be good, in view of the next result. 
In the following, the point $v$ need not be an extreme point; it is, however, in a proper face of $K$.

Lemma 7.11. Suppose $K$ is a simplex and set $Z=K \cap \mathcal{L}$, where $\mathcal{L}$ is a closed flat. Suppose in addition that there exists a closed face, $F$, having more than one extreme point, such that $F \cap Z=\emptyset$, and a point $v$ in $K \backslash Z$ from which there exist two distinct line segments to $F$, both of which pass through $Z$. Then $Z$ is not good.

In particular, this applies if there exists a face $F$ of nonzero dimension such that $Z \cap F=\emptyset$ and $Z$ contains an interior point of $K$.

Proof. Since $F \cap Z$ is empty, $F$ is a closed face, and $Z$ is compact, there exists $b_{1} \in$ Aff $K$ such that $b_{1} \geq 0, b_{1} \mid F \equiv 0$, and $b_{1} \mid Z \gg 1$. Let $x_{1}$ and $x_{2}$ be the endpoints of the line segments in $F$ (joining $F$ to $v$ ), and let $z_{1}, z_{2}$ be the intersection points of the two lines with $Z$. We can write $z_{i}=\lambda_{i} x_{i}+\left(1-\lambda_{i}\right) v$ for some $\lambda_{i} \in(0,1)$. By interchanging $x_{1}$ with $x_{2}$ if necessary, we may assume that

$$
\frac{\lambda_{2}\left(1-\lambda_{1}\right)}{\lambda_{1}\left(1-\lambda_{2}\right)} \leq 1
$$

We may also find $a_{1} \in$ Aff $K$ such that $a_{1} \geq 0, a_{1}\left(x_{1}\right) \geq 3 / 4,0<a_{1}\left(x_{2}\right)<3 / 4-3 \epsilon$ for some $0<\epsilon<\min \left\{b_{1}(k)-1 \mid k \in Z\right\}$ and $a_{1} \mid Z \leq 1$. Hence $b:=b_{1}+\epsilon \mathbf{1}$ and $a:=a_{1}+(\epsilon / 2) 1$ satisfy $a, b \gg 0$ and $b|Z \gg a| Z$.

Suppose that $Z$ is good (for Aff $K$ ). Then there would exist $c \in$ Aff $K$ such that $0 \leq c \leq b$ and $c|Z=a| Z$. Let $K^{\prime}$ denote the triangle with vertices $\left\{x_{1}, x_{2}, v\right\}$ and let $Z^{\prime}$ be $K^{\prime} \cap Z$, which is easy to see is just the line segment joining $z_{1}$ to $z_{2}$. Then $c\left|Z^{\prime}=a\right| Z^{\prime}$ and $0 \ll c\left|K^{\prime} \ll b\right| K^{\prime}$. We show this is impossible. Denote $v$ by $x_{0}$.

Since $z_{i}=\lambda_{i} x_{i}+\left(1-\lambda_{i}\right) x_{0}(i=1,2)$, on writing $c\left(x_{i}\right)=a\left(x_{i}\right)-\delta_{i}(i=0,1,2)$, we find $0=\lambda_{i} \delta_{i}+\left(1-\lambda_{i}\right) \delta_{0}(i=1,2)$, whence $\delta_{i}=-\left(1-\lambda_{i}\right) \delta_{0} / \lambda_{i}(i=1,2)$. The condition that $c\left(x_{i}\right)>0$ yields three inequalities for $\delta_{0}$, computed from $\delta_{i}<a\left(x_{i}\right)$ :

$$
\max \left\{-\frac{\lambda_{1} a\left(x_{1}\right)}{\left(1-\lambda_{1}\right)},-\frac{\lambda_{2} a\left(x_{2}\right)}{\left(1-\lambda_{2}\right)}\right\}<\delta_{0}<a\left(x_{0}\right) .
$$

The conditions $c\left(x_{i}\right)<b\left(x_{i}\right)$ yield three more inequalities,

$$
a\left(x_{0}\right)-b\left(x_{0}\right)<\delta_{0}<\min \left\{\frac{\left(b\left(x_{1}\right)-a\left(x_{1}\right)\right) \lambda_{1}}{1-\lambda_{1}}, \frac{\left(b\left(x_{2}\right)-a\left(x_{2}\right)\right) \lambda_{2}}{1-\lambda_{2}}\right\} .
$$

Combining all of them, we see that

$$
\begin{aligned}
\max & \left\{a\left(x_{0}\right)-b\left(x_{0}\right),-\frac{\lambda_{1} a\left(x_{1}\right)}{1-\lambda_{1}},-\frac{\lambda_{2} a\left(x_{2}\right)}{1-\lambda_{2}}\right\} \\
& <\min \left\{a\left(x_{0}\right), \frac{\left(b\left(x_{1}\right)-a\left(x_{1}\right)\right) \lambda_{1}}{1-\lambda_{1}}, \frac{\left(b\left(x_{2}\right)-a\left(x_{2}\right)\right) \lambda_{2}}{1-\lambda_{2}}\right\} .
\end{aligned}
$$

In particular,

$$
\begin{aligned}
b\left(x_{1}\right) & >a\left(x_{1}\right)-\frac{\lambda_{2}\left(1-\lambda_{1}\right)}{\left(1-\lambda_{2}\right) \lambda_{1}} a\left(x_{2}\right) \\
& \geq a\left(x_{1}\right)-a\left(x_{2}\right)>\frac{3}{4}-\left(\frac{3}{4}-3 \epsilon\right)=3 \epsilon .
\end{aligned}
$$

This contradicts $b\left(x_{1}\right)=\epsilon$. 
Lemma 7.11 applies if $K$ is finite dimensional and $Z=K \cap \mathcal{L}$ is a codimension one simplex that misses at least three vertices and is not a face (for then the hyperflat must separate one of the vertices from the other two). In particular, if the hyperflat misses all the vertices, $Z$ is not good. It also applies for most (but not all) $Z$ that are line segments from one facet to another that miss all the vertices of $K$ (exceptions occur when the endpoints are in faces complementary to each other).

Here is an easy construction of a good $Z=K \cap \mathcal{L}$ that is neither a face nor a singleton, corresponding to the coproduct of two good subsets (for a discussion of coproducts of simplices in the category of simplices, see [Goo86, p 164]). Let $K$ be a simplex, and let $F$ and $F^{\prime}$ be disjoint closed faces. Select $W$, a subset of $F$ that is good with respect to $F$, and $W^{\prime}$ a subset of $F^{\prime}$ that is good with respect to $F^{\prime}$. Let $Z \equiv Z\left(W, F ; W^{\prime}, F^{\prime}\right) \subset K$ be the convex hull of $W \cup W^{\prime}$ inside $K$. The convex hull of $F \cup F^{\prime}$ will be denoted $F \vee F^{\prime}$, and is itself a face of $K$.

It is completely routine to verify that this construction yields good $Z$. If $a, b$ are strictly positive elements of Aff $K$ such that $a|Z \ll b| Z$, then of course $a|W \ll b| W$ and $a\left|W^{\prime} \ll b\right| W^{\prime}$, so by goodness of $W, W^{\prime}$ relative to $F, F^{\prime}$ respectively, there exist strictly positive $d \in \operatorname{Aff} F$ and $d^{\prime} \in$ Aff $F^{\prime}$ such that $d|W=a| W, d^{\prime} \mid W^{\prime}=$ $a \mid W^{\prime}$, and $d \ll b\left|F, d^{\prime} \ll b\right| F^{\prime}$. Since $F$ and $F^{\prime}$ are disjoint and closed, there exists $c \in \operatorname{Aff}\left(F \vee F^{\prime}\right)$ such that $c \mid F=d$ and $c \mid F^{\prime}=d^{\prime}$, and it is obvious that $0 \ll c\left|\left(F \vee F^{\prime}\right) \ll b\right|\left(F \vee F^{\prime}\right)$ and $c|Z=a| Z$. Since $F \vee F^{\prime}$ is itself a closed face of $K$, we may apply the extension result for closed faces to find $c^{\prime} \ll b$ such that $c^{\prime}\left|\left(F \vee F^{\prime}\right)=c\right|\left(F \vee F^{\prime}\right)$.

This generalizes the two-dimensional situation, wherein $K$ is a triangle and $Z$ is the line segment from a vertex to a point on the opposite edge. This allows us to obtain building blocks for good subsets of $K$. The building blocks are singleton subsets in the boundary and closed faces (disjoint from the face containing the singleton). The process can be iterated finitely many times (or simply deal with finitely many disjoint faces), but it is not clear that we can take the closure of the increasing union if infinitely many disjoint faces or singleton sets are involved.

Thus a line segment in the triangle from a vertex is the coproduct of a zerodimensional face (the vertex) and a point in the complementary edge. The smallest example for which we can construct a coproduct of two singletons that hits no vertices occurs in a tetrahedron. Let $K$ be such, with vertices $\left\{x_{0}, x_{1}, x_{2}, x_{3}\right\}$. Let $z_{1}$ be a point in the relative interior of the edge $F$ joining $x_{0}$ to $x_{1}$, and choose $z_{2}$ in the relative interior of the edge $F^{\prime}$ joinining $x_{2}$ to $x_{3}$. The construction yields a good line segment joining $z_{1}$ to $z_{2}$; it contains no vertices of $K$.

On the other hand, if the two building blocks are both faces, then the construction yields only a face. In particular, this means that in any sequence of building blocks (closed faces and singleton sets), we can combine all the closed faces in one (the closure of the convex hull of a union of faces is a face), and then we are simply adding singleton sets in disjoint faces (which have to be complementary to the previous ones).

It is also routine to verify that if $W$ and $W^{\prime}$ are intersections of $F$ and $F^{\prime}$ respectively with flats, then the resulting $Z\left(W, F ; W^{\prime}, F^{\prime}\right)$ is also of this form; that is, the closure of the affine span of $Z$ intersected with $K$ is just $Z$.

Conjecture. If $K$ is a simplex and $Z=K \cap \mathcal{L}$ where $\mathcal{L}$ is a closed flat, then $Z$ is good as a set of traces on Aff $K$ (with the strict ordering) if and only if $Z$ is obtained as the closure of an increasing union of $Z_{i}=K \cap \mathcal{L}_{i}$, where each $Z_{i}$ is obtained as a coproduct of $Z_{i-1}$ with a building block. 
If $K$ is finite dimensional, the conjecture is almost certainly true, and we can just forget the limit process.

Corollary 7.12. Suppose that $(R, 1)$ is a partially ordered ring that is a simple dimension group, with 1 as an order unit. Then every subset of $\partial_{e} S(R, 1)$ is refinable. In particular, every pure trace is refinable.

Remark 1. Returning to Example 10, where $R=\mathbf{Q}[x]$ with the strict ordering obtained from restriction to an interval $I \subseteq[a, b]$, we saw that the pure trace $\phi_{\alpha}$ ( $\alpha \in I$ ) is good if and only if $\alpha$ is algebraic and no other algebraic conjugates of $\alpha$ are in $I$, and $\phi_{\alpha}$ is bad if and only if $\alpha$ is transcendental. Both of these types of pure traces are obviously refinable, and the remaining pure traces $\left(\phi_{\alpha}\right.$, where $\alpha$ is algebraic and at least one other algebraic conjugate is in $I$ ) are neither good nor bad (nor ugly), but are refinable by this result.

Remark 2. The conclusion does not hold if we weaken the hypotheses to some of their consequences; for example, that $(G, u)$ be a simple dimension group for which $S(G, u)$ is a Bauer simplex or even finite dimensional. The simple dimension group $G^{\prime}$ of Example 11 (below) has a pure trace which is not refinable (nor ugly), but the pure trace space consists of three points. On the other hand, for simple dimension groups with exactly two traces, modulo the infinitesimals, every pure trace is either good, bad (hence refinable), or ugly (hence not refinable).

Proof. If $R=\mathbf{Z}$, the only trace is obviously good, hence refinable. Otherwise, $R$ has dense image in its affine representation. Let $U$ be a set of pure normalized traces (hence multiplicative). We show by routine arguments that $Z \equiv Z(\operatorname{ker} U)$ is a face of $S(R, 1)$ and then the image of $\operatorname{ker} U$ is dense in $Z(\operatorname{ker} U)^{\perp}$. Then by Proposition 7.7, $Z$ is order unit good. As $R$ is simple as a dimension group, $Z$ is good; therefore $Z$ is refinable, and thus $U$ is refinable.

Let $\tau$ be an element of $U$; since $\tau$ is pure, it is multiplicative (Proposition 3.1), hence $\operatorname{ker} \tau$, and therefore $\operatorname{ker} U$, is an ideal. Let $X=\partial_{e} S(R, 1)$; this is a compact space (since $R$ is a partially ordered ring with 1 as order unit), and we can identify $C(X, \mathbf{R})$ with $\operatorname{Aff}(S(R, 1)$ ) (and the probability measure space on $X$ with $S(R, 1)$ ). The closure, $J$ of $\operatorname{ker} U$ is a closed ideal of the closure of $R$, that is, of $C(X, \mathbf{R})$. Hence there exists compact $Y \subset X$ such that $J=$ Ann $Y:=\{f \in C(X, R)|f| Y \equiv 0\}$. There exists a unique face $F$ of $S(R, 1)$ such that $Y=F \cap X$ (always true for Bauer simplices), and it is easy to check that Ann $Y=F^{\perp}$.

Now $Z(\operatorname{ker} U)$ consists of normalized traces (that is, probability measures on $X$ ) that kill ker $U$, so that $F \subseteq Z$. On the other hand, if $\sigma \in Z(\operatorname{ker} U)$, then $\sigma(J)=0$, so $\sigma \in F^{\perp}$.

Example 11. Simple dimension groups with three pure traces; pure traces which are refinable but not good, etc.

Let $\alpha$ and $\beta$ be positive irrational numbers, and define the following four elements of $\mathbf{R}^{3}$ :

$$
\begin{aligned}
& v_{1}=\left(\begin{array}{lll}
0 & 0 & 1
\end{array}\right), \\
& v_{1}^{\prime}=\left(\begin{array}{lll}
0 & \beta & 1
\end{array}\right), \\
& v_{2}=\left(\begin{array}{lll}
\alpha & 1 & 0
\end{array}\right), \\
& v_{3}=\left(\begin{array}{lll}
1 & 0 & 0
\end{array}\right) .
\end{aligned}
$$


Let $G$ be the rational vector space spanned by $\left\{v_{1}, v_{2}, v_{3}\right\}$ and let $G^{\prime}$ be the rational vector space spanned by $\left\{v_{1}^{\prime}, v_{2}, v_{3}\right\}$. Let $\tau_{i}$ denote the projections on the $i$ th coordinate. Each of $G$ and $G^{\prime}$ is dense in $\mathbf{R}^{3}$, so equipped with the strict ordering inherited from $\mathbf{R}^{3}$, each is a simple dimension group, and $\left\{\tau_{i}\right\}$ is the set of pure traces (in both cases).

For $G$, we consider $U=\left\{\tau=\tau_{1}\right\}$. It is a pure trace whose kernel is spanned (as a rational vector space) by $v_{1}$, and since $\tau_{2}\left(v_{1}\right)=0$, it follows that $\operatorname{ker} \tau$ is not dense in $(\operatorname{ker} \tau)^{\vdash} \cong \mathbf{R}^{2}$. Hence $\tau$ is not good; neither is it bad, since $v_{1} \notin \operatorname{Inf}(G)=\{0\}$. On the other hand, $Z(\operatorname{ker} U)$ is the face spanned by $\tau_{1}$ and $\tau_{2}$, and is thus good (with respect to Aff $S(G, u)$ ). From Lemma 7.10, $Z(\operatorname{ker} U$ ) is good (with respect to $G$ ), therefore refinable, and thus $\tau$ is refinable; this also follows from an (easy) construction of coproducts below. In this case, $\tilde{U}=\left\{\tau_{1}\right\} \neq Z(\operatorname{ker} U)$.

In contrast, for $G^{\prime}$, again let $\tau=\tau_{1}$. Its kernel is $v_{1}^{\prime} \mathbf{Q}$, so this is a pure trace that is neither bad nor good. However, it isn't refinable either, because $\tau_{2}-\beta \tau_{3}$ kills $v_{1}^{\prime}$ (and thus ker $\tau$ ), but there is no trace $\sigma=r \tau_{2}+s \tau_{3}$ (where $r$ and $s$ are nonnegative real numbers) that kills $v_{1}^{\prime}$, so that $Z(\operatorname{ker} \tau)=\{\tau\}$ (in this case, $Z=Z(\operatorname{ker} \tau)$ is a face), and thus $Z^{\vdash}$ is two dimensional, but $\operatorname{ker} \tau$ is rank one, so the image of $\operatorname{ker} \tau$ is not dense in $Z^{\perp}$. (Since $G^{\prime}$ is a rational vector space, there is no difference between $Z(\operatorname{ker} U)$ being good or refinable.)

For both groups, $\tau_{2}$ is refinable but neither good nor bad, and $\tau_{3}$ is good (by the density criterion).

An easy sufficient condition for refinability comes from the following construction, similar to the one constructing good sets out of the building blocks of faces and singletons. Just as the ordered tensor product is the product within the category of simple dimension groups with order unit (and also in the category of dimension groups with order unit), we can find a coproduct in this category (which differs from the coproduct in dimension groups, that is, the direct sum), provided we exclude the cyclic case (ordered groups isomorphic to $\mathbf{Z}$ ). Let $G$ and $H$ be noncyclic simple dimension groups. Form the direct sum, $\mathcal{C} \equiv \mathcal{C}(G, H)=G \oplus H$, and impose on it the strict ordering; that is,

$$
\mathcal{C}^{+} \backslash\{(0,0)\}=\left\{(g, h) \in G \oplus H \mid g \in G^{++}, h \in H^{++}\right\} .
$$

It is easy to verify that $\mathcal{C}$ is simple and that the Riesz interpolation and unperforation properties hold (the former uses the strict interpolation property that holds for simple noncyclic dimension groups); thus $\mathcal{C}$ is a simple dimension group. The pure trace space of $\mathcal{C}$ is the disjoint union of the pure trace space of $G$ and of $H$ (the pure traces are precisely those of the form $\tau \oplus 0$ and $0 \oplus \sigma$ where $\tau$ varies over the pure traces of $G$ and $\sigma$ varies over the pure traces of $H$ ). Note however, the inclusions $G \rightarrow G \oplus\{0\} \subset \mathcal{C}$ and $H \rightarrow\{0\} \oplus H \subset \mathcal{C}$ are not order preserving. Instead, the projection maps $\mathcal{C} \rightarrow G$ and $\mathcal{C} \rightarrow H$ are. It is routine to verify that within the category of simple noncyclic dimension groups, this is the coproduct. [We have to exclude $\mathbf{Z}$ since, as is well known, no noncyclic simple dimension group can have a discrete trace, which is what would happen if we allow one of $G$ or $H$ to be cyclic - so the constructed group would not be a dimension group.]

This is a special case of a pullback of simple dimension groups (in Mat02, Matui discusses pushouts of dimension groups; these are dual to pullbacks). In this more general setting, we have two positive onto maps $\phi: G \rightarrow K$ and $\psi: H \rightarrow K$, where 
$K$ is a dimension group (possibly zero), and we form $\mathcal{C}=\{(g, h) \mid \phi(g)=\psi(h)\} \subseteq$ $G \oplus H$, with the strict ordering induced by the inclusion. For the resulting subgroup to be a dimension group, we require that if $\rho$ is a pure trace of $K$, then $\rho \circ \phi$ and $\rho \circ \psi$ are pure traces of their respective groups, or zero. The extremal trace space is then obtained by taking the union and identifying the traces that factor through the map to $K$. If $K$ is zero, we obtain $\mathcal{C}(G, H)$.

Lemma 7.13. Suppose $G$ and $H$ are noncyclic simple dimension groups, and $\tau$ is a pure refinable (good) trace of $G$. Then the extension of $\tau$ to a pure trace of $\mathcal{C}(G, H)$ given by $\tau \oplus 0$ (that is, $(g, h) \mapsto \tau(g))$ is refinable (good).

Proof. Fix an order unit $u$ (with respect to which $\tau$ is refinable) of $G$, and let $v$ be any order unit of $H$. Let $T$ denote $\tau \oplus 0$. Obviously $T([(0,0),(u, v)])=\tau([0, u])$, and if nonzero $a \in[0, u] \backslash\{u\}$ and we pick any element $v^{\prime} \in[0, v] \backslash\{0, v\}$ (such exist since $H$ is noncyclic), then $\left[a, v^{\prime}\right] \in[(0,0),(u, v)]$. It easily follows that refinability (goodness) of $T$ holds.

Referring back to Example 11, we can quickly show (again) that the trace $\tau_{1}$ of $G$ is refinable. Let $G_{0}=\mathbf{Q} v_{3}+\mathbf{Q} v_{2}$; equipped with the strict ordering as a subgroup of $\mathbf{R}^{2}$ (i.e., stripping the third coordinate), $G_{0}$ is a simple dimension group and the first coordinate projection, $p$, is a pure trace that is bad (since $\alpha$ is irrational), hence refinable. Now $G$ may be identified in the obvious way with $\mathcal{C}\left(G_{0}, \mathbf{Q}\right)$ (where $v_{3}$ yields the rational direct summand) and $p \oplus 0=\tau_{1}$, so the latter is refinable, by Lemma 7.13 .

\section{Appendix A. TEnsor PRODUCTS DyNAMiCALly}

If $(X, S)$ is a minimal zero-dimensional dynamical system (that is, $X$ is a Cantor set and $S$ is a self-homeomorphism), then the partially ordered group

$$
\mathrm{K}_{0}\left(C(X, \mathbf{C}) \times{ }_{S} \mathbf{Z}\right):=\mathrm{K}_{0}(X, S)
$$

has a natural pre-ordering (as all $\mathrm{K}_{0}$-groups do), which makes it into a simple dimension group, alternatively described by the quotient ordering on the cokernel of I $-S: C(X, \mathbf{Z}) \rightarrow C(X, \mathbf{Z})$. The image of the constant function 1 is the order unit with respect to which traces are normalized, and there is a natural affine homeomorphism between the invariant probability measures on $X$ and the normalized traces on the dimension group. Conversely, every simple dimension group arises in this fashion, even when we include a specific order unit as part of the data. The simple dimension group is a complete invariant for strong orbit equivalence. This and much more is due to GPS95.

If we take two dimension groups with order unit, $(G, u)$ and $(H, v)$, it is a natural construction to form the ordered tensor product, as in GH86]: on the tensor product over $\mathbf{Z}$ (that is, as abelian groups), $G \otimes_{\mathbf{Z}} H$ (the subscript $\mathbf{Z}$ is usually deleted), impose the positive cone generated by all pure tensors, $g \otimes h$, where $g \in G^{+}$and $h \in H^{+}$. That this is a partially ordered group is not entirely obvious in general, but for dimension groups, it is even a dimension group (from EHCLS80]), with $u \otimes v$ as designated order unit. If both $G$ and $H$ are simple, then so is $G \otimes H$.

The tensor product construction on dimension groups is perfectly natural, so at least in the case that the dimension groups are simple, they should correspond to some construction on the minimal dynamical systems that yielded them. Explicitly, if $(X, S)$ and $(Y, T)$ are minimal zero-dimensional dynamical systems corresponding 
respectively to simple dimension groups with order units, $(G, u)$ and $(H, v)$, then what minimal action does $(G \otimes H, u \otimes v)$ correspond to?

We answer this; however, the answer is not entirely satisfactory, since it only yields one of the many possible minimal $\mathbf{Z}$-actions on $X \times Y$ (necessarily orbit equivalent to the obvious $\mathbf{Z}^{2}$-action), and there is no obvious way to single out a natural one.

We do this by exploiting (as is done in GPS95]) Vershik's adic maps.

Pick a Bratteli diagram that represents $(G, u)$ (typically, at level zero, these have a single point at the apex). Let $X_{n}$ be the space of paths of length $n$ beginning at the top. This is a finite space, and we may form the path space, $X=\lim _{\leftarrow} X_{n}$, where the maps $\pi_{n}: X_{n+1} \rightarrow X_{n}$ are given by simply removing the last vertex and edge. Two paths are tail equivalent if they are composed of the same edges, with up to finitely many differences.

On each $X_{n}$, we define an equivalence relation $R_{n}$ simply by declaring two paths equivalent if they end at the same vertex. On each $R_{n}$-equivalence class, we try to impose a total ordering, $\prec_{n}$, in such a way that if the finite paths, given as sequences of edges, $p:=\left(e_{1}, e_{2}, \ldots, e_{n}\right) \prec_{n} q=\left(f_{1}, f_{2}, \ldots, f_{n}\right)$, then for every admissible edge $g_{n+1}$, the new paths satisfy $\left(p, g_{n+1}\right) \prec_{n+1}\left(q, g_{n+1}\right)$. If this is done, we can compare any two infinite paths that are tail equivalent to each other, inducing a partial ordering $\prec$ on $X$, and it is easy to check that if $p$ is an infinite path which is not maximal, it has an immediate successor, i.e., there exists $p^{\prime}$ such that $p \prec p^{\prime}$, and if $p \prec p^{\prime \prime}$ with $p \neq p^{\prime \prime}$, then $p^{\prime} \prec p^{\prime \prime}$.

Let $\mathcal{M}^{+}$and $\mathcal{M}^{-}$denote the collections of maximal and minimal paths respectively; both are compact. Vershik's adic map is given by $p \mapsto p^{\prime}$, defined on $X \backslash \mathcal{M}^{+}$ with range in $X \backslash \mathcal{M}^{-}$. As observed in GPS95], if $\mathcal{M}^{+}$and $\mathcal{M}^{-}$each consist of a single point, then the adic map can be extended to a minimal homeomorphism of $X$ by sending the maximal path to the minimal one.

To construct a minimal dynamical system that yields the tensor product of the dimension groups, we deal with the orderings a bit more generally. Let $(W, \prec)$ be a partially ordered set; an equivalence relation $R$ on $W$ is said to be compatible with $\prec$ if for elements $x, y$, and $z$, whenever $y R z$ and either $x \prec y$ or $y \prec x$, then $x R z$. For example, if $R$ is the trivial equivalence relation ( $x R y$ for all $x$ and $y$ ), this obviously holds, but it also holds if $R$ is the transitive closure of the relation given by $x \prec y$ or $y \prec x$. In fact, the latter is the minimal equivalence relation compatible with $\prec$. (It may turn out to be trivial, e.g., if $W$ has a minimal element less than or equal to every element of $W$.) Even better, the relation $x \prec y$ or $y \prec x$ could turn out to be an equivalence relation, and this is what happens in the motivating example.

If $(W, \prec, R)$ and $\left(Z, \prec^{\prime}, S\right)$ are partially ordered sets with respective compatible equivalence relations, then we can construct a new partially ordered set (with a compatible equivalence relation), which we call the $R$-lexicographic product, defined as follows. The underlying set is $W \times Z$, the equivalence relation is $R \times S$, and the ordering is given by

$$
(w, z) \prec^{\prime \prime}\left(w_{1}, z_{1}\right) \text { when } \begin{cases}z \prec^{\prime} z_{1} & \text { if } z \neq z_{1} \& w R w_{1}, \text { or } \\ w \prec w_{1} & \text { if } z=z_{1} .\end{cases}
$$

It is routine to verify that $W \times Z$ becomes a poset (this does not require the equivalence relation $S$ ) and, moreover, $R \times S$ is compatible with respect to $\prec$ ". We 
abbreviate this new object, $W \times_{R \text {-lex }} Z$. If $R$ is the trivial equivalence relation, then this is the usual lexicographic product.

In our situation, arising from Bratteli diagrams, we simply take $X_{n} \times_{R_{n} \text {-lex }} Y_{n}$, and then the new ordered compactum is $\lim _{\leftarrow} X_{n} \times{ }_{R_{n} \text {-lex }} Y_{n}$, with the products of the usual maps. Since inverse limits commute with finite direct products, the underlying toplogical space is just $X \times Y$; however, the ordering does not commute with inverse limits.

Now it is routine to check that the transition matrices (that is, obtained from $\left.X_{n+1} \times_{R_{n+1} \text {-lex }} Y_{n+1} \rightarrow X_{n} \times_{R_{n} \text {-lex }} Y_{n}\right)$ are exactly the matrix tensor products of the transition matrices; this is, if $\pi_{n}$ is induced by $M_{n}$ (a rectangular matrix with entries from the positive integers) and $N_{n}$ for $X_{n+1} \rightarrow X_{n}$ and $Y_{n+1} \rightarrow Y_{n}$ respectively, then the transition matrix on the new construction is simply $M_{n} \otimes N_{n}$ (the Kronecker product, in old-fashioned terminology).

Again it is routine to verify that the unique successor property holds, and so the adic map is defined on an open subset. Furthermore, the collection of maximal paths of the new thing is just the cartesian product, $\mathcal{M}_{X}^{+} \times \mathcal{M}_{Y}^{+}$, and the same applies to the minimal paths. Hence if each ordered Bratteli diagram just has one maximal and one minimal path, then so does the new object, and thus we create a minimal homeomorphism, call it $S \otimes T$, on an ordered Bratteli diagram, whose dimension group is the tensor product of the original dimension groups. Unfortunately, this is not canonical, in that we make many choices on how to order the product spaces; we picked the $R_{n}$-lexicographical order because it seemed reasonable.

The effect of the adic map in this construction is to sometimes choose $S \times 1$ and other times choose $1 \times T$, acting on a path. Hence to each element of $X \times Y$ we can associate an element of $\{0,1\}^{\mathbf{N}}$; its coordinate in the $n$th position will be a 0 if the $n$th iterate of the path under the $\mathbf{Z}$ action is given by $S \times 1$, otherwise the entry will be one. This gives a factor map from $S \otimes T$ to a subshift of the two-shift.

It is known that any product action of a finite number of minimal homeomorphisms is hyperfinite; that is, it is orbit equivalent to a single homeomorphism. The following observation exhibits this minimal homeomorphism explicitly. Let $B$ and $B^{\prime}$ be two Bratteli diagrams corresponding to simple dimension groups $G$ and $G^{\prime}$ respectively. Let $S$ and $T$ be minimal Vershik homeomorphisms defined on the path spaces of $B$ and $B^{\prime}$. Then the sets of ergodic (that is, extremal) invariant measures, $M(X, S)$ and $M\left(X^{\prime}, T\right)$, are naturally in bijection wtih $\partial_{e} S(G, u)$ and $\partial_{e} S\left(G^{\prime}, u^{\prime}\right)$ respectively. Construct the Bratteli diagram $B \otimes B^{\prime}$ and the Vershik map $S \otimes T$ acting on its path space as above. It is well known that the product action of $\mathbf{Z}^{2}$ on $X \times X^{\prime}$ generated by $S \times \mathrm{I}$ and $\mathrm{I} \times T$ is orbit equivalent to a $\mathbf{Z}$-action by a single minimal homeomorphism, say $V$. Then $V$ and $S \otimes T$ have the same sets of invariant ergodic probability measures, namely $M(X, S) \times M\left(X^{\prime}, T\right) \cong \partial_{e} S\left(G \otimes G^{\prime}, u \otimes u^{\prime}\right)$. Hence $S \otimes T$ is orbit equivalent to $V$, and therefore $S \otimes T$ is orbit equivalent to the $\mathbf{Z}^{2}$-action. This also applies to product $\mathbf{Z}^{n}$-actions, by induction.

The following is presumably well known; its proof is standard in this context.

Lemma A.1. Let $(X, S)$ and $(Y, T)$ be compact zero-dimensional dynamical systems, and let $\nu$ be a probability measure on $X \times Y$ extremal among measures invariant under the action of $\mathbf{Z}^{2}$ generated by $S \times 1$ and $1 \times T$. Then there exist an extremal $S$-invariant probability measure $\mu_{1}$ on $X$ and an extremal $T$-invariant probability measure $\mu_{2}$ on $Y$ such that $\nu=\mu_{1} \times \mu_{2}$ (the product measure). 
Proof. Define $\mu_{1}$ and $\mu_{2}$, measures on $X$ and $Y$ respectively, via $\mu_{1}(A)=\nu(A \times Y)$ and $\mu_{2}(B)=\nu(X \times B)$. These are invariant under $S$ and $T$ respectively, and their extremality (within the set of invariant measures) follows from the corresponding property of $\nu$.

For each clopen set $B$ of $Y$ such that $q:=\nu(X \times B) \neq 0$ (that is, $\mu_{2}(B) \neq 0$ ), define a measure on $X, \mu_{B}$, via $\mu_{B}(A)=\nu(A \times B) / \mu_{2}(B)$ (for $A$ an arbitrary clopen of $X)$. It is routine that this is an $S$-invariant measure, and $\mu_{B}(A) \leq \frac{1}{q} \mu_{1}(A)$ for all clopen sets $A$, and thus for all elements in the $\sigma$-algebra they generate. By extremality of $\mu_{1}$, we must have $\mu_{B}=\mu_{1}$. Hence $\nu(A \times B) / \mu_{2}(B)=\mu_{1}(A)$; that is, $\nu(A \times B)=\mu_{1}(A) \mu_{2}(B)$ for all clopen sets $B$ such that $\mu_{2}(B) \neq 0$.

If $\mu_{2}(B)=0$, then $\nu(A \times B) \leq \nu(X \times B)=0$; thus for all clopen sets $B$ in $Y$, we have $\nu(A \times B)=\mu_{1}(A) \mu_{2}(B)$. Let $\nu^{\prime}$ be the product measure on $X \times Y$ obtained from $\mu_{1}$ and $\mu_{2}$. Then $\nu$ agrees with $\nu^{\prime}$ on rectangles; since the rectangles generate the full $\sigma$-algebra generated by the clopen sets in the product, we must have $\nu=\nu^{\prime}=\mu_{1} \times \mu_{2}$.

Corollary A.2. Let $(X, S)$ and $(Y, T)$ be zero-dimensional compact dynamical systems, and form the space $(X \times Y,\langle S \times 1,1 \times T\rangle)$ (the natural action of $\mathbf{Z}^{2}$-not of $\mathbf{Z}$ (via $S \times T)$-on $X \times Y$ ). If $\alpha$ and $\beta$ are pure invariant measures on $X \times Y$ whose corresponding traces agree on $K_{0}\left(C(X \times Y) \times_{S, T} \mathbf{Z}^{2}\right)$, then $\alpha=\beta$.

Proof. Since $\alpha$ and $\beta$ are pure invariant measures, by the preceding, there exist $\mu_{i}, \nu_{i}$, pure invariant measures on the relevant sets such that $\alpha=\mu_{1} \times \nu_{1}$ and $\beta=\mu_{2} \times \nu_{2}$. If $\mu_{1} \neq \mu_{2}$, then there exists a clopen $A$ of $X$ such that $\mu_{1}(A) \neq \mu_{2}(A)$, so that $\mu_{1}\left(\chi_{A}\right) \neq \mu_{2}\left(\chi_{A}\right)$, and thus the corresponding traces on $\mathrm{K}_{0}\left(C(X) \times_{S} \mathbf{Z}\right)$ have different values at the equivalence class $\left[\chi_{A}\right]$. The natural map $\mathrm{K}_{0}\left(C(X) \times_{S}\right.$ $\mathbf{Z}) \otimes \mathrm{K}_{0}\left(C(Y) \times_{T} \mathbf{Z}\right) \rightarrow \mathrm{K}_{0}\left(C(X \times Y) \times{ }_{\langle S, T\rangle} \mathbf{Z}^{2}\right)$ is order preserving (but not ontothere is a free rank one contribution from $\left.\mathrm{K}_{1} \otimes \mathrm{K}_{1}\right)$, and $\alpha(A \times Y) \neq \beta(A \times Y)$ entails that the corresponding traces on $\mathrm{K}_{0}$ of the $\mathbf{Z}^{2}$ crossed product disagree on $\left[\chi_{A \times Y}\right]$. Hence $\mu_{1}=\mu_{2}$, and similarly, $\nu_{1}=\nu_{2}$, so that $\alpha=\beta$.

\section{APPENDix B. UnPERFORATION OF QUOTIENTS}

Unperforation of quotients by convex subgroups (of the form ker $U$ ) seems to be a significant contributor to the difference between goodness (of sets of traces) and refinability, as in Proposition 7.6(b) and (f), so it seemed worthwhile to investigate this in more detail. First, we give sufficient conditions for unperforation, generalizing from the divisible case. Then we show that many quotients $G / \operatorname{ker} U$ are very badly not unperforated.

Here is a sufficient condition for $G / L$ to be unperforated, where $L$ is a convex subgroup of $G$ such that $G / L$ is torsion-free. It is likely necessary as well in this context (simple dimension groups).

Proposition B.1. Let $(G, u)$ be a simple dimension group, and let $L$ be a convex subgroup of $G$ such that $G / L$ is torsion-free (as an abelian group). Sufficient for $G / L$ to be unperforated with respect to the quotient ordering is the following:

$(*)$ For all $k \in L$, for all $\epsilon>0$, there exist $k_{1}, k_{2}, k_{3} \in L$ such that

$$
\widehat{k}=2 \widehat{k}_{1}+\widehat{k}_{2}-\widehat{k}_{3} \quad \text { and } \quad \widehat{k}_{2}, \widehat{k}_{3} \geq-\epsilon \mathbf{1} .
$$

Remark. The coefficient 2 of $k_{1}$ can obviously be replaced by any integer exceeding 1 . 
Remark. The ostensibly weaker condition,

$\left(\frac{*}{2}\right)$ For all $k \in L$, for all $\epsilon>0$, there exist $k_{1}, k_{2}, k_{3} \in L$ such that

$$
\left\|\widehat{k}-\left(2 \widehat{k}_{1}+\widehat{k}_{2}-\widehat{k}_{3}\right)\right\|<\frac{\epsilon}{2} \quad \text { and } \quad \widehat{k}_{2}, \widehat{k}_{3} \geq-\frac{\epsilon}{2} \mathbf{1}
$$

is equivalent to $(*)$, since we can incorporate the difference, $k-\left(2 k_{1}+k_{2}-k_{3}\right)$, into either $k_{2}$ or $k_{3}$.

Proof. Since $G$ is simple, so is $G / L$ (with the quotient ordering); thus for $a$ in $G / L$, $P(a)$ (defined as before as $\left\{n \in \mathbf{N} \mid n a \in(G / L)^{++}\right\}$) is either empty, all of $\mathbf{N}$ (so $a \geq 0$ ), or cofinite. If the last holds, there exists a positive integer $l$ such that $2^{l} a \geq 0$. Hence it suffices to show that if $a$ is in $G / L$ and $2 a \geq 0$, then $a \geq 0$.

To this end, suppose $a=g+L$ and $2 a \geq 0$. Then there exist $k \in L$ and $p \in G^{++}$ such that $2 g+k=p$. Select $\epsilon<\inf _{\sigma \in S(G, u)} \sigma(p) / 2$, and apply $\left(^{*}\right)$ to $k$. Then $2 g+2 k_{1}+k_{2}=p+k_{3}$; define $h=g+k_{1}+k_{2}$ so that $2 \widehat{h}=2\left(\widehat{g}+\widehat{k}_{1}+\widehat{k}_{2}\right)=$ $\widehat{p}+\widehat{k}_{2}+\widehat{k}_{3}$. Hence $2 \widehat{h} \geq \widehat{p}-2 \epsilon \mathbf{1} \gg 0$. Thus $\widehat{h} \gg 0$, so that $h$ is an order unit, and $h+L=g+L=a$. So $a \in(G /:)^{++}$.

If $J$ is a closed subgroup of a Banach space, then the divisible subgroup of $J$ (the maximal rational subspace of $J$ ), $D(J)$, is a closed real vector space and the maximal real vector subspace of $J$. For $J$ a closed subgroup of Aff $K$, let $\mathcal{P}(J)$ be the subgroup of $J$ generated by $\{j \in J \mid \geq 0\}$. An elementary condition for $J$ to be a real vector space (that is, $J=D(J)$ ) when $J$ arises as the closure of the image of $\operatorname{ker} U$ (for $U$ a subset of $S(G, u)$ ), is that for $k \in \operatorname{ker} U$, the element $\widehat{k} / 2 \in \operatorname{Aff} S(G, u)$ can be uniformly approximated by images of elements of $\operatorname{ker} U$.

If $J=\mathcal{P}(J)$, then $Z(\operatorname{ker} J)$ is a closed face of $K$. If we take critical simple dimension groups of the form $G=\left\langle e_{i} ; \sum \alpha_{i} e_{i}\right\rangle$ (where $\left\{1, \alpha_{1}, \alpha_{2}, \ldots\right\}$ is linearly independent over the rationals), and set $U=\left\{\tau_{1}\right\}$ (consisting of the the trace obtained from the projection on the first coordinate), then $\operatorname{ker} U=\left\langle e_{i}\right\rangle_{i \neq 1}$. Each $\widehat{e}_{i}=e_{i}$ (a consequence of the notation), so is nonnegative as a function, and thus $J=\mathcal{P}(J)$ and it follows that the quotient group is $\mathbf{Z}+\alpha_{1} \mathbf{Z}$ with the ordering inherited from the reals.

On the other hand, if we alter this somewhat, there is a drastic change. Take $G=\left\langle e_{1}, e_{2} ;(\alpha, \beta)\right\rangle$ with $\{1, \alpha, \beta\}$ linearly independent over the rationals, so that $G$ is dense in $\mathbf{R}^{2}$. Set $\tau=\tau_{1}+\tau_{2}:(a, b) \mapsto a+b$; then $\operatorname{ker} \tau=(1,-1) \mathbf{Z}$. It turns out that $H=G / \operatorname{ker} \tau$ is very holey.

For example, we can construct for every positive integer $m$ an element $a_{m}$ in $H$ such that $P\left(a_{m}\right)=\mathbf{N} \backslash\{1,2,3, \ldots, m\}$ (that is, $n a_{m} \in G^{+}$if and only if $n \geq m+1$ ). To see this, by density of $G$ in $\mathbf{R}^{2}$, we may find $A_{m}=(r, s) \in G$ such that $1 /(m+1)<r<1 / m$ and $-1 /(2 m+1)<s<-1 /(2 m+2)$. Then $(m+1) A_{m}+$ $(1,-1) \gg 0$, but for no $j<m+1$ does there exist an integer $n$ (positive or negative) such that $j A_{m}+(n,-n) \gg 0$. Hence if $a_{m}=A_{m}+\operatorname{ker} \tau$, then $P\left(a_{m}\right) \cap$ $\{1,2, \ldots, m+1\}=\{m+1\}$, and if $l>m$, then $l \in P\left(a_{m}\right)$.

It is easy to describe the ordering on the quotient: $(r, s)$ has positive image if and only if both $r+s>0$ and the interval $[-s, r]$ contains an integer. In particular, if $r+s>1$, then $(r, s)$ has positive image in $G / \operatorname{ker} \tau$. It is plausible that as $a$ varies over the elements of $H$, the corresponding $P(a)$ run over all cofinite subsemigroups of $\mathbf{N}$. It would be interesting to decide whether interpolation holds for this example.

Corollary B.2. Let $L$ be a subgroup of the simple dimension group $(G, u)$ such that $L \cap G^{+}=\{0\}$ and $G / L$ is torsion-free. Let $J$ denote the closure of the image 
of $L$ in $\operatorname{Aff} S(G, u)$. Either of the following is sufficient for $G / L$ to be unperforated with respect to the quotient ordering:

(a) $J$ is a real vector space.

(b) $D(J)+\mathcal{P}(J)$ is dense in $J$.

Proof. In both cases, $\left(\frac{*}{2}\right)$ applies; in the first case, $k_{2}=k_{3}=0$.

Unbelievably, there is an example wherein Proposition B.1 applies but Corollary B.2 does not. This was originally constructed as a potential example of a singleton set $U$ that was refinable and not good with $U=Z(\operatorname{ker} U$ ) (no such examples are known); however, it turned out not to be refinable, as $G / \operatorname{ker} U$ is unperforated but ker $U$ does not have dense range in $Z(\operatorname{ker} U)^{\vdash}$.

Example 12. A simple dimension group with four pure traces together with a singleton set $U=\{\tau\}$ such that $G / \operatorname{ker} U$ is unperforated, but does not satisfy either sufficient condition of Corollary B.2.

Let $\{a, b, c, r, s\}$ be an algebraically independent set of real numbers (this is likely overkill). Define the following six elements of $\mathbf{R}^{4}$ :

$$
\begin{aligned}
& v_{1}=\left(\begin{array}{llll}
0 & 0 & 1 & -1
\end{array}\right), \\
& v_{2}=\left(\begin{array}{llll}
0 & 0 & s & -s
\end{array}\right), \\
& v_{3}=\left(\begin{array}{cccc}
1 & -1 & a & 0
\end{array}\right), \\
& v_{4}=\left(\begin{array}{cccc}
r & -r & 0 & b
\end{array}\right), \\
& v_{5}=\left(\begin{array}{llll}
1 & 0 & 1 & 1
\end{array}\right), \\
& v_{6}=\left(\begin{array}{llll}
c & 1 & 0 & 0
\end{array}\right) .
\end{aligned}
$$

Let $G$ be the subgroup of $\mathbf{R}^{4}$ generated by $\left\{v_{i}\right\}$. Let $\tau_{j}(j=1,2,3,4)$ be the coordinate projections, and set $\tau=\tau_{1}+\tau_{2}$. Then $\tau^{\vdash}=\{(x,-x, y, z) \mid x, y, z \in \mathbf{R}\}$.

We first observe that $\operatorname{ker} \tau=\left\langle v_{1}, v_{2}, v_{3}, v_{4}\right\rangle$ (an easy consequence of linear independence of $\{1, r, c\}$ over the rationals). Now let $J$ be the closure of $\operatorname{ker} \tau$. We immediately see that $v_{1} \mathbf{R} \subset J$ (from linear indepence of $\{1, s\}$ over $\mathbf{Q}$ ). Thus $J$ is the closure of the span of $\left\{v_{1} \mathbf{R}, v_{3}, v_{4}^{\prime}:=(r,-r,-b, 0)\right\}$. We note that $\left\{v_{1}, v_{3}, v_{4}\right\}$ is a real basis for $\tau^{\vdash}$. Hence $J=v_{1} \mathbf{R}+v_{3} \mathbf{Z}+v_{4}^{\prime} \mathbf{Z}$, expressed as a direct sum of a vector space and the discrete abelian group $\left\langle v_{3}, v_{4}\right\rangle$. In particular, $J \cong \mathbf{R} \oplus \mathbf{Z}^{2}$.

Now we show $G$ is dense in $\mathbf{R}^{4}$. Since $J$ is in the closure of $G$, we can add elements of $v_{1} \mathbf{R}$ to the generators of $G$ without affecting its closure. This shows that the elements $v_{4}^{\prime}$ and $v_{5}^{\prime}=(1,0,2,0)$ are in $J$. Now consider the subgroup of $\mathbf{R}^{3} \times\{0\}$ generated by $v_{3}, v_{4}^{\prime}, v_{5}^{\prime}, v_{6}$; delete the terminal zero of each, and calculate all four $3 \times 3$ determinants arising from this set of four vectors. The result is a linearly independent set over $\mathbf{Q}$ (a consequence of algebraic independence of $\{a, b, c, r\}$ ). Hence this subgroup is dense in $\mathbf{R}^{3} \times\{0\}$. Since $v_{1} \mathbf{R}+\left(\mathbf{R}^{3} \times\{0\}\right)=\mathbf{R}^{4}, G$ is dense in $\mathbf{R}^{4}$.

Hence $G$, equipped with the strict ordering, is a simple dimension group. Let $U=$ $\{\tau\}$, so $\operatorname{ker} U=\operatorname{ker} \tau$. Now $\mathcal{P}(J)=\{0\}$. This is obvious from the decomposition $J=v_{1} \mathbf{R} \oplus v_{3} \mathbf{Z} \oplus v_{4}^{\prime} \mathbf{Z}$, and since $D(J)=v_{1} \mathbf{R}$, the criterion in the corollary cannot be applied. We show that the hypothesis of Proposition B.1 does apply.

Select $k \in \operatorname{ker} \tau$. If $k$ has a nonzero component in $\left\langle v_{1}, v_{2}\right\rangle$, we can immediately reduce to the zero component there. Hence we can write $k=t v_{3}+u v_{4}=$ $(t a+u r,-(t a+u r), t u, u b)$ for integers $t, u$. Since $\{1, r\}$ is linearly independent 
over $\mathbf{Q}$, there exist integers $t^{\prime}, u^{\prime}$ such that $\left|(t+u r) / 2-\left(t^{\prime}+u^{\prime} r\right)\right|<\epsilon / 8$. Set $k^{\prime}=t^{\prime} v_{3}+u^{\prime} v_{4}$, so that $k-2 k^{\prime}=\left(2 \delta,-2 \delta,\left(t-2 t^{\prime}\right) a,\left(u-2 u^{\prime}\right) b\right)$ where $|\delta|<\epsilon / 8$. Next, we may find integers $m, n$ such that $\left|m+n s-\left(t-2 t^{\prime}\right) a / 2\right|<\epsilon / 8$. Set $k^{\prime \prime}=m v_{1}+n v_{2}$, so that $k-2 k^{\prime}-2 k^{\prime \prime}=\left(2 \delta,-2 \delta, 2 \eta,\left(u-2 u^{\prime}\right) b-2(m-n s)\right):=k^{\prime \prime \prime}$ where $|\eta|<\epsilon / 8$.

Let $f$ denote the fourth coordinate of $k^{\prime \prime \prime}$. Set $k_{1}=k^{\prime}+k^{\prime \prime}$. If $f \geq 0$, then $k^{\prime \prime \prime} \geq \min \{-2|\delta|,-2|\eta|\}$, so we can set $k_{2}=k-2 k^{\prime}-2 k^{\prime \prime}$ and $k_{3}=0$; if $f^{\prime}<0$, set $k_{3}=-k^{\prime \prime \prime}$ and $k_{2}=0$.

Hence $G / \operatorname{ker} \tau$ is unperforated. It is easy to see that $Z(\operatorname{ker} \tau)=\{\tau\}$, so $G / \operatorname{ker} \tau$ has unique trace, that induced by $\tau$. Being simple, unperforated, and with unique trace, $G / \operatorname{ker} \tau$ is order isomorphic to the subgroup $\tau(G)$ of the reals (with the relative ordering), in particular, $G / \operatorname{ker} \tau \cong \mathbf{Z}+c \mathbf{Z} \subset \mathbf{R}$.

In this example, and since $J \neq \tau^{\vdash}$ (as $J$ is not a real vector space), $\tau$ is not good; since $G / \operatorname{ker} \tau$ is unperforated, $Z(\operatorname{ker} \tau)$ is not refinable, and therefore $\tau$ is not refinable. Since $\operatorname{ker} \tau$ contains a real basis for $\tau^{\vdash}, \tau \otimes 1$ is a good trace of $G \otimes \mathbf{Q}$.

Now we analyze properties of the individual pure traces and their finite subsets. Among the cases considered, only one of the quotients $G / \operatorname{ker} U$ is holey (not unperforated). This is probably atypical.

$U=\left\{\tau_{1}\right\}$ We note that $\operatorname{ker} \tau_{1}=\left\langle v_{1}, v_{2}, v_{5}-v_{3}\right\rangle$; the closure is $v_{1} \mathbf{R} \oplus\left(v_{5}-v_{3}\right) \mathbf{Z}$, and it is easy to check that regardless of the value of $a, J=\mathcal{P}(J)$, and thus by Corollary B.2(b), $G / \operatorname{ker} \tau_{1}$ is unperforated. However, $\operatorname{ker} \tau_{1}$ does not contain a real basis for $\tau_{1}^{\perp}$, so even $\tau_{1} \otimes 1$ on $G \otimes \mathbf{Q}$ is not refinable.

$U=\left\{\tau_{2}\right\}$ We have ker $\tau_{2}=\left\langle v_{1}, v_{2}, v_{3}+v_{6}, v_{5}\right\rangle ;$ here $J \cong \mathbf{R} \oplus \mathbf{Z}^{2}$ and $J=\mathcal{P}(J)$, so Corollary B.2(b) applies again, and thus $G / \operatorname{ker} \tau_{2}$ is unperforated. As in the case with $\tau, \tau_{2}$ is not refinable.

$U=\left\{\tau_{3}\right\}$ or $\left\{\tau_{4}\right\}$ We note $\operatorname{ker} \tau_{3}=\left\langle v_{4}, v_{6}, v_{5}-v_{1}\right\rangle$ (since $\{1, a, s\}$ is rationally independent). Here $\operatorname{ker} \tau_{3}$ is discrete and contains a basis, so $\tau_{3}$ is ugly. It is routine to check that once again $J=\mathcal{P}(J)$, so the quotient is unperforated, and thus $\tau_{3}$ is not refinable. Similar computations reveal that $\tau_{4}$ has the same properties.

$U=\left\{\tau_{1}, \tau_{2}\right\}$ Then $J=v_{1} \mathbf{R}$, so $J=\mathcal{D}(J)$ and thus $G / \operatorname{ker} U$ is unperforated. In this case, $Z(\operatorname{ker} U)$ is the face spanned by $U$, and since $\operatorname{ker} U$ is not dense in $U^{\perp} \cong \mathbf{R}^{2}, U$ is not good (or refinable).

$U=\left\{\tau_{2}, \tau_{3}\right\}$ Then ker $U=\left\langle v_{5}-v_{1}\right\rangle$, which is discrete of rank one, hence $U$ is not refinable; however, $J=\operatorname{ker} U=\mathcal{P}(J)$, so $G / \operatorname{ker} U$ is unperforated.

$U=\left\{\tau_{3}, \tau_{4}\right\}$ Then ker $U=\left\langle v_{6}\right\rangle$, again discrete. Here $Z$ (ker $\left.U\right)$ is again the affine span of $U$, and it follows from the rank of $\operatorname{ker} U$ that $U$ is not refinable. However, there are now two cases to consider.

If $c>0$, then $J=\mathcal{P}(J)$, so $G / \operatorname{ker} U$ is unperforated. On the other hand, if $c<0$, there is a trace in the interior of the trace space that kills $\operatorname{ker} U$, and by Proposition B.4 below, $G / \operatorname{ker} U$ is not unperforated.

$U=\left\{\tau_{2}, \tau_{4}\right\}$ We see $\operatorname{ker} U=\left\langle v_{6}+v_{3}, v_{5}+v_{1}\right\rangle$, which is discrete and equal to $\mathcal{P}(J)$. Hence $\operatorname{ker} U$ is unperforated, and thus $U$ is not refinable; here $U$ is ugly.

$U=\left\{\tau_{1}, \tau_{3}\right\}$ or $\left\{\tau_{1}, \tau_{4}\right\}$ We observe that $\operatorname{ker} U=\{0\}$, and thus $Z(\operatorname{ker} U)=K$, hence $U$ is refinable. 
If $G$ is a simple dimension group and $L$ is a quotientable subgroup, then it is routine that $G / L$ is a dimension group if and only if $G / L$ is unperforated and $Z(L)$ is a simplex. In particular, if $J=\mathcal{P}(J)$, then $Z(L)$ is actually a face of the trace space, hence a simplex. The following is a slight improvement.

It is also a slightly more general statement than assuming $G$ has a stronger ordering in which it is still a dimension group, but for which $L$ is an order ideal. The candidate for the stronger ordering is typically the coordinatewise one (that is, first factor out the infinitesimal subgroup, and say $g \geq^{\prime} 0$ if $\widehat{g} \geq 0$; however, with respect to this ordering, $G$ does not always have interpolation).

We can strengthen the result on quotients by taking out one of the terms in $(*)$.

Proposition B.3. Let $(G, u)$ be a simple dimension group and $L$ be a convex subgroup of $G$ with the following property:

for all $l \in L$ and all $\epsilon>0$, there exist $l_{1}, l_{2} \in L$ such that $l=$ $l_{1}-l_{2}$ and $\widehat{l}_{i} \geq-\epsilon \mathbf{1}$.

Then $G / L$ is a dimension group. In particular, this applies if $J=\mathcal{P}(J)$, where $J$ is the closure of $L$ in the affine representation.

Proof. We know $G / L$ satisfies unperforation by Proposition B.1, so it suffices to prove Riesz decomposition holds. Pick $A, B, C$ in $(G / L)^{+}$such that $A \leq B+C$. If any of $A, B, C, A+B-C$ is zero, there is nothing to do. Otherwise, we can lift each of $A, B, C$ to order units, $a, b, c$ respectively. Then there exists $p \in G^{++}$and $l$ in $L$ such that $b+c-a=p+l$.

Select $\epsilon<\min \{\inf \widehat{p}, \inf \widehat{c}\}$. Applying the property with this choice of $\epsilon$, we have $b+\left(c+l_{2}\right)-a=\left(p+l_{1}\right)$; by the choice of $\epsilon$, each of the parenthesized terms is an order unit, and thus we may apply Riesz decomposition in $G$ to $a \leq b+\left(c+l_{2}\right)$. This yields a corresponding decomposition in $G / L$.

In contrast, here is a reasonably general result on the failure of unperforation for quotients by convex subgroups. It does not require simplicity, merely approximate divisibility. Compare with Lemma 7.11.

Proposition B.4. Suppose $(G, u)$ is an approximately divisible dimension group, with normalized trace space $K=S(G, u)$. Let $U$ be a subset of $K$ for which $Z=$ $Z(\operatorname{ker} U)$ satisfies $Z \cap \operatorname{Int}(K) \neq \emptyset$. If there exists a trace $\tau$ such that $\tau(\operatorname{ker} U)$ is nonzero and cyclic, then $G / \operatorname{ker} U$ is not unperforated.

Remark. The condition that $Z$ meet the interior of $K$ obviously rules out faces; however, there are good $Z$ (with respect to Aff $K$ ) that contain an interior point.

Remark. The proof actually exhibits a great deal of holeyness among the potential order units.

Proof. There exists $z \in Z$ and in the interior of $K$; hence the line segment joining $\tau$ to $z$ extends outside $Z$ but still within $K$. The affine span of this segment hits the boundary of $K$ at $\tau_{2} \in \partial K$ (neither $\tau_{2}$ nor $\tau$ need be pure). We thus have a decomposition $z=\lambda \tau+(1-\lambda) \tau_{2}$ for some $0<\lambda<1$. There exists $\delta \neq 0$ such that $\tau(\operatorname{ker} \tau)=\delta \mathbf{Z}$; without loss of generality, we may assume $\delta>0$. For any $v \in \operatorname{ker} U$, $z(v)=0$, so that $\tau_{2}(v)=-\lambda \tau(v) /(1-\lambda)$; in particular, $\tau_{2}$ restricted to $\operatorname{ker} U$ has discrete (and nonzero) range. 
Fix an element $k \in \operatorname{ker} U$ such that $\tau(k)=\delta$. Since $\tau_{2}(k)<0, k$ is not positive. Now we find $g$ in $G$ such that $g+k \gg 0$ but $\tau(g)<0$. By density of the range of $G$ in Aff $K$, there exists $j \gg 0$ such that $0 \ll \widehat{j} \ll(\delta / 2) \mathbf{1}$, and set $g=j-k$. Then $\tau(g)=\tau(j)-\delta<-\delta / 2$.

As $\tau_{2}(k)<0$ and $g+k$ is an order unit, it follows that $\tau_{2}(g)>0$. If $m$ is a sufficiently large positive integer, then

$$
\frac{|\tau(g)|}{m}<\frac{\delta}{3} \quad \text { and } \quad \frac{\tau_{2}(g)}{m}<\frac{\lambda \delta}{3(1-\lambda)} .
$$

Choose positive real $\epsilon$ small enough such that

$$
\epsilon<\min \left\{\frac{m \delta}{2}, \frac{m \lambda \delta}{2(1-\lambda)}, \frac{|\tau(g)|}{2}, \frac{\tau_{2}(g)}{2}, \min _{\sigma \in K} \sigma(g+k)\right\} .
$$

Again using density of $G$ in Aff $K$, we may find $g_{1} \in G$ such that $\left\|\widehat{g}_{1}-\widehat{g} / m\right\|<\epsilon / m$. Then $\left\|m \widehat{g}_{1}+\widehat{k}-(\widehat{g}+\widehat{k})\right\|<\epsilon<\min _{\sigma \in K} \sigma(g+k)$. Hence $m g_{1}+k \gg 0$, so $m g_{1}+k$ is an order unit in $G$, and thus $m g_{1}+\operatorname{ker} U$ is an order unit (in particular, it is positive) in $G / \operatorname{ker} U$.

Now we show $g_{1}+\operatorname{ker} U$ is not in the positive cone of $G / \operatorname{ker} U$. Suppose to the contrary that it is. If $b:=g_{1}+k_{1}$ is a positive element of $G$ but not an order unit for some $k_{1} \in \operatorname{ker} U$, we obtain an immediate contradiction: for no positive integer $l$ can $u \leq l b$, but this contradicts $m b$ being an order unit. Hence $g_{1}+k_{1} \gg 0$. There exists an integer $n$ (possibly negative) such that $\tau\left(k_{1}\right)=n \delta$; then $\tau_{2}\left(k_{1}\right)=-\lambda n \delta /(1-\lambda)$. Applying $\tau$ and $\tau_{2}$ to $g_{1}+k_{1}$, we obtain

$$
\tau\left(g_{1}\right)+n \delta>0 \quad \text { and } \quad \tau_{2}\left(g_{1}\right)-\frac{n \lambda \delta}{(1-\lambda)}>0 .
$$

Since $0<\lambda<1$ and $\delta>0$, this yields

$$
\frac{\tau_{2}\left(g_{1}\right)(1-\lambda)}{\lambda \delta}>n>-\frac{\tau\left(g_{1}\right)}{\delta} .
$$

Since $\left\|\widehat{g}_{1}-\widehat{g} / m\right\|<\epsilon / m$, it follows that

$$
\left|\tau_{2}\left(g_{1}\right)-\tau_{2}(g) / m\right|<\epsilon / m \text { and }\left|\tau\left(g_{1}\right)-\tau(g) / m\right|<\epsilon / m .
$$

The latter yields $\tau\left(g_{1}\right)<\tau(g) / m+\epsilon / m<\tau(g)(1-1 / 2) / m<0$, and the former yields

$$
\tau_{2}\left(g_{1}\right)<\frac{\epsilon}{m}+\frac{\tau_{2}(g)}{m}<\frac{\epsilon}{m}+\frac{\lambda \delta}{3(1-\lambda)}<\frac{5}{6} \frac{\lambda \delta}{1-\lambda} .
$$

Therefore $n<5 / 6$, so $n \leq 0$. However, $-\tau\left(g_{1}\right)>0$, so $n>0$, a contradiction.

Altering some of the numbers can increase the set of integers $r$ such that $r g_{1}+$ $\operatorname{ker} U$ is not positive.

By similar methods, we can at least obtain a necessary condition for refinable sets, which together with the previous results is tantalizingly close to the conjectural result that refinability of $U$ implies $G / \operatorname{ker} U$ is unperforated (which would imply that $Z(\operatorname{ker} U)$ is good).

Proposition B.5. Suppose that $(G, u)$ is an approximately divisible dimension group, and $U$ is a refinable subset of $S(G, u)$. If $\sigma$ is a trace of $G$, then either $\sigma(\operatorname{ker} U)$ is zero or dense in $\mathbf{R}$. 
Proof. If not, there exists $\tau \in S(G, u)$ such that $\tau(\operatorname{ker} U)=\delta \mathbf{Z}$ for some positive real number $\delta$. Select $k \in \operatorname{ker} U$ such that $\tau(k)=\delta$. There exist (lots of) order units $v$ such that $v \gg k$ (any sufficiently large integer multiple of any order unit will satisfy this); pick one of them, call it $v$, and suppose $\tau(v) / \delta=r>0$. Select an integer $N>r$. Now choose positive $\epsilon$ such that

$$
\epsilon<\min \left\{(N-r) \delta, \inf _{\sigma \in S(G, u)} \sigma(v), \inf _{\sigma \in S(G, u)} \sigma(v-k)\right\} .
$$

By density of the image of $G$ in $\operatorname{Aff} S(G, u)$, we may find $v^{\prime}$ in $G$ such that $\left\|\widehat{v}^{\prime}-\widehat{v} / N\right\|<\epsilon / N$. For any trace $\sigma, \sigma\left(v^{\prime}\right)>(\sigma(v)-\epsilon) / N>0$. Hence $v^{\prime}$ is an order unit. In addition, $\sigma\left(N v^{\prime}-k\right)>\sigma(v-k)-\epsilon>0$, so that $N v^{\prime}-k$ is also an order unit. Moreover, $\tau\left(v^{\prime}\right)<(\tau(v)+\epsilon) / N=(r \delta+\epsilon) / N<\delta$. Define $a_{i}=v^{\prime}$ for $i=1,2, \ldots, N$ and set $b=N v^{\prime}-k$. Then $b=\sum a_{i}-k$, where $b, a_{i}$ are all order units and $k \in \operatorname{ker} U$. By refinability, there exist $c_{i} \in G^{+}$such that $c_{i}-v^{\prime}=k_{i} \in \operatorname{ker} U$ and $b=\sum c_{i}$. Applying $\tau$, we have the following (with integer $n(i))$ :

$$
\begin{aligned}
\tau\left(c_{i}\right) & =\tau\left(v^{\prime}\right)+\tau\left(k_{i}\right)=\tau\left(v^{\prime}\right)+n(i) \delta, \\
N \tau\left(v^{\prime}\right)-\delta & =\tau(b)=\sum \tau\left(c_{i}\right) .
\end{aligned}
$$

This yields $\sum n(i)=-1$. Hence at least one of the $n(i)$, call it $n(j)$, is less than or equal to -1 . However, $\tau\left(c_{j}\right)=\tau\left(v^{\prime}\right)+n(j) \delta \leq \tau\left(v^{\prime}\right)-\delta<0$, contradicting positivity of $c_{j}$.

Corollary B.6. Let $(G, u)$ be a simple dimension group with finitely many pure traces, and let $U$ be a refinable subset of $S(G, u)$ such that any one of the following conditions holds:

(i) $Z \equiv Z(\operatorname{ker} U)$ is a face;

(ii) $Z$ contains an interior point of $K=S(G, u)$;

(iii) $Z(\operatorname{ker} U)$ is contained in a facet, but contains a relative interior point of that facet;

(iv) the smallest face containing $U, F$, is good for $G$.

Then $Z(\operatorname{ker} U)$ is good.

Remark. The hypothesis in (iv) that $F$ be a good subset for $G$ is not entirely satisfactory. What would be preferable is merely that $F$ be refinable (which would imply that $Z(\operatorname{ker} F)$ - which can strictly contain $F$ even though the latter is a faceis good). However, we were not able to prove this. Likely sufficient is that $F$ (the face generated by $U$ ) be of codimension one in a good face.

Proof. Since $U$ is refinable for $G$, so is it refinable for $G^{\prime}=G \otimes \mathbf{Q}$. In this case, $G^{\prime} / \operatorname{ker}_{G^{\prime}} U$ is unperforated (since it is a rational vector space), and since $Z(\operatorname{ker} U$ ) is refinable for $G^{\prime}$, from Proposition 7.6(e), the image of $\operatorname{ker}_{G^{\prime}} U=(\operatorname{ker} U) \otimes \mathbf{Q}$ is dense in $Z^{\vdash}$.

Let $J$ denote the closure of the image of $\operatorname{ker} U$. Since the pure trace space is finite dimensional, $J$ can be written as $D(J) \oplus W$, where $W$ is a discrete abelian group (therefore, free). Since $J \mathbf{R}=Z^{\vdash}$, any $\mathbf{Z}$-basis for $W$ together with a real basis for the real vector space $D(J)$ will constitute a real basis for $Z^{\vdash}$. By Proposition 7.6(f), it suffices to show that $G / \operatorname{ker} U$ is unperforated. 
(i) Now suppose $Z$ is a face. We claim that $W \subseteq P(J)$. The set of elements of $Z^{\perp}$ that are nonnegative (as functions on $S(G, u)$ ) forms a closed cone in $Z^{\perp}$ that contains an open ball (true for any closed face). By taking a ball of sufficiently large radius inside this cone, we can guarantee that it contains a fundamental parallelotope of $W$, that is, a $\mathbf{Z}$-basis of $W$. Thus $W \subseteq P(J)$.

Hence $J=D(J)+\mathcal{P}(J)$, so by Corollary B.2, $G / \operatorname{ker} U$ is unperforated, and this case is done.

(ii) Now assume that $Z$ contains an interior point, $z$, of $K=S(G, u)$. If $W$ is not zero, there exists a bounded linear functional on Aff $K, \rho$, such that $\rho(\operatorname{ker} \tau)=\delta \mathbf{Z}$ for some $\delta>0$. Since $z$ is an interior point of $K$, there exists a positive integer $N$ such that $\tau:=\rho+N z$ is a trace (a positive linear combination of the pure traces). Clearly $\tau(\operatorname{ker} U)=\rho(\operatorname{ker} \tau)$, so $\tau$ has nonzero cyclic image. By Proposition B.5, this contradicts refinability of $U$. Hence $W=\{0\}$ and thus $J$ is a real vector space. By Corollary B.2, $G / \operatorname{ker} U$ is unperforated.

(iii) Let the facet be given by vertices $\left\{\tau_{0}, \ldots, \tau_{n-1}\right\}$, and let $\tau_{n}$ denote the complementary vertex. We can write $\sigma=\sum_{i=0}^{n} r_{i} \tau_{i}$. The relative interior point of the facet belonging to $Z(\operatorname{ker} U)$ is written in the form $\phi=\sum_{i=0}^{n-1} R_{i} \tau_{i}$ where $R_{i}>0$.

By multiplication by -1 if necessary, we may assume $r_{n} \geq 0$. Then there exists $N$ such that all the coefficients of $\tau:=\sigma+N \phi$ are nonnegative, and thus $\tau$ is a trace. Since $\phi(\operatorname{ker} U)=0$, it follows that $\tau(\operatorname{ker} U)=\mathbf{Z} \delta$. Since $\tau$ is a trace, Proposition B.5 applies, contradicting refinability. Hence the discrete part of $J$ is zero, so $J$ is divisible; thus (as it contains a real basis for $\operatorname{ker} U^{\vdash}$ and is complete), $J=(\operatorname{ker} U)^{\vdash}$, and so $Z(\operatorname{ker} U)$ is good.

(iv) This is by induction on the dimension of $K$. If $F$ is $K$ or a facet, this is covered by (ii) or (iii).

Since $F$ is good, $G_{1}:=G / \operatorname{ker} F$ is itself a simple dimension group, and, of course, its trace space is naturally homeomorphic to $F$. Now $\operatorname{ker} F \subseteq \operatorname{ker} U$; let $V$ be the image of $\operatorname{ker} U$ in $G_{1}$ (it is $\operatorname{ker} U$ relative to $G_{1}$ ). Then $G / \operatorname{ker} U \cong G_{1} / V$. If $a_{i}, b \in G_{1}^{+}$, and $b=\sum a_{i}+k$ with $k \in V$, we can lift (since ker $F$ is good) each to $A_{i}, B \in G^{+}$such that $A_{i}+V=a_{i}$ and $B+V=b$, and then $B-\left(\sum A_{i}\right) \in \operatorname{ker} U$. By refinability of $U$, there exist $A_{i}^{\prime} \in G^{+}$such that $A_{i}^{\prime}-A_{i} \in \operatorname{ker} U$ and $B=\sum A_{i}^{\prime}$. Setting $a_{i}^{\prime}=A_{i}^{\prime}+V$, we see that $U$ is refinable for the dimension group $G / \operatorname{ker} F$.

We may replace $U$ by $Z(\operatorname{ker} U) \cap K$; the latter is compact convex, and since $F$ is the smallest face generated by $U, L(U)$ contains a point in the relative interior of $F$. Since $U$ is refinable relative to $G_{1}$, now Corollary B.6(ii) applies, so that $Z(\operatorname{ker} U)$ is good with respect to $G_{1}$. Thus $G_{1} / V$ is unperforated, and because of the order isomorphism with $G / \operatorname{ker} Z(\operatorname{ker} U)$, the latter is unperforated. Finally, by Proposition 7.6(f), $U$ refinable and $G / \operatorname{ker} U$ unperforated imply $Z(\operatorname{ker} U)$ is good.

Combining this with the earlier results, we see that when $G$ has finitely many pure traces and $Z(\operatorname{ker} U)$ is a face or contains an interior point, then $U$ is refinable if and only if $\operatorname{ker} U$ has dense image in $Z(\operatorname{ker} U)^{\vdash}$. In particular, if $\tau$ is a trace for which we could verify that $Z(\operatorname{ker} \tau)$ is a face, then $\tau$ is refinable if and only if $\operatorname{ker} \tau$ is dense $Z(\operatorname{ker} \tau)^{\vdash}$. This excludes all the pure traces of our now standard example, $G=\left\langle e_{i} ; \sum \alpha_{i} e_{i}\right\rangle$, from being refinable. The first case for which the conjecture that refinability of $U$ implies goodness of $Z(\operatorname{ker} U)$ is unknown occurs when $U$ is a singleton in the relative interior of an edge of a tetrahedron. 


\section{REFERENCES}

[Aki99] Ethan Akin, Measures on Cantor space, Proceedings of the 14th Summer Conference on General Topology and its Applications (Brookville, NY, 1999), Topology Proc. 24 (1999), no. Summer, 1-34 (2001). MR1876365(2002j:28002)

[Aki05] Ethan Akin, Good measures on Cantor space, Trans. Amer. Math. Soc. 357 (2005), no. 7, 2681-2722 (electronic), DOI 10.1090/S0002-9947-04-03524-X. MR2139523 (2006e:37003)

[ADMY08] Ethan Akin, Randall Dougherty, R. Daniel Mauldin, and Andrew Yingst, Which Bernoulli measures are good measures?, Colloq. Math. 110 (2008), no. 2, 243-291, DOI 10.4064/cm110-2-2. MR2353909 (2009f:37009)

[Alf71] Erik M. Alfsen, Compact convex sets and boundary integrals, Springer-Verlag, New York-Heidelberg, 1971. Ergebnisse der Mathematik und ihrer Grenzgebiete, Band 57. MR0445271 (56 \#3615)

[AE80] L. Asimow and A. J. Ellis, Convexity theory and its applications in functional analysis, London Mathematical Society Monographs, vol. 16, Academic Press, Inc. [Harcourt Brace Jovanovich, Publishers], London-New York, 1980. MR623459 (82m:46009)

[Aus07] Tim D. Austin, A pair of non-homeomorphic product measures on the Cantor set, Math. Proc. Cambridge Philos. Soc. 142 (2007), no. 1, 103-110, DOI 10.1017/S0305004106009741. MR2296394 (2008g:28017)

[BK11] S. Bezuglyi and O. Karpel, Homeomorphic measures on stationary Bratteli diagrams, J. Funct. Anal. 261 (2011), no. 12, 3519-3548, DOI 10.1016/j.jfa.2011.08.009. MR2838033(2012i:37001)

[BK00] Sergey Bezuglyi and Jan Kwiatkowski, The topological full group of a Cantor minimal system is dense in the full group, Topol. Methods Nonlinear Anal. 16 (2000), no. 2, 371-397. MR1820514 (2002b:37012)

[BKMS10] S. Bezuglyi, J. Kwiatkowski, K. Medynets, and B. Solomyak, Invariant measures on stationary Bratteli diagrams, Ergodic Theory Dynam. Systems 30 (2010), no. 4, 973-1007, DOI 10.1017/S0143385709000443. MR2669408 (2012g:37019)

[BH94] Mike Boyle and David Handelman, Entropy versus orbit equivalence for minimal homeomorphisms, Pacific J. Math. 164 (1994), no. 1, 1-13. MR.1267499(95m:28019)

[BH96] Mike Boyle and David Handelman, Orbit equivalence, flow equivalence and ordered cohomology, Israel J. Math. 95 (1996), 169-210, DOI 10.1007/BF02761039. MR1418293 (98a:46082)

[BH3] Mike Boyle and David Handelman, unpublished drafts, 1993-2011.

[DMY07] Randall Dougherty, R. Daniel Mauldin, and Andrew Yingst, On homeomorphic Bernoulli measures on the Cantor space, Trans. Amer. Math. Soc. 359 (2007), no. 12, 6155-6166, DOI 10.1090/S0002-9947-07-04352-8. MR2336321 (2009f:37010)

[Eff81] Edward G. Effros, Dimensions and $C^{*}$-algebras, CBMS Regional Conference Series in Mathematics, vol. 46, Conference Board of the Mathematical Sciences, Washington, D.C., 1981. MR623762 (84k:46042)

[EHCLS80] Edward G. Effros, David E. Handelman, and Chao Liang Shen, Dimension groups and their affine representations, Amer. J. Math. 102 (1980), no. 2, 385-407, DOI 10.2307/2374244. MR.564479 (83g:46061)

[GPS95] Thierry Giordano, Ian F. Putnam, and Christian F. Skau, Topological orbit equivalence and $C^{*}$-crossed products, J. Reine Angew. Math. 469 (1995), 51-111. MR $1363826(97 \mathrm{~g}: 46085)$

[GPS99] Thierry Giordano, Ian F. Putnam, and Christian F. Skau, Full groups of Cantor minimal systems, Israel J. Math. 111 (1999), 285-320, DOI 10.1007/BF02810689. MR.1710743 (2000g:46096)

[GW95] Eli Glasner and Benjamin Weiss, Weak orbit equivalence of Cantor minimal systems, Internat. J. Math. 6 (1995), no. 4, 559-579, DOI 10.1142/S0129167X95000213. MR1339645 (96g:46054)

[Goo86] K. R. Goodearl, Partially ordered abelian groups with interpolation, Mathematical Surveys and Monographs, vol. 20, American Mathematical Society, Providence, RI, 1986. MR845783 (88f:06013) 
[GH80] K. R. Goodearl and D. E. Handelman, Metric completions of partially ordered abelian groups, Indiana Univ. Math. J. 29 (1980), no. 6, 861-895, DOI 10.1512/iumj.1980.29.29060. MR.589651 (82b:06020)

[GH86] K. R. Goodearl and D. E. Handelman, Tensor products of dimension groups and $K_{0}$ of unit-regular rings, Canad. J. Math. 38 (1986), no. 3, 633-658, DOI 10.4153/CJM1986-032-0. MR845669 (87i:16043)

[GH82] K. R. Goodearl and D. E. Handelman, Stenosis in dimension groups and $A F C^{*}$ algebras, J. Reine Angew. Math. 332 (1982), 1-98. MR656856 (83m:46101)

[Han82a] David Handelman, Extensions for $A F C^{*}$ algebras and dimension groups, Trans. Amer. Math. Soc. 271 (1982), no. 2, 537-573, DOI 10.2307/1998898. MR654850 (84e:46063)

[Han82b] David Handelman, Free rank $n+1$ dense subgroups of $R^{n}$ and their endomorphisms, J. Funct. Anal. 46 (1982), no. 1, 1-27, DOI 10.1016/0022-1236(82)90041-6. MR654462 (84h:06019)

[Han86] David Handelman, Imitation product-type actions on UHF algebras, J. Algebra 99 (1986), no. 1, 1-21, DOI 10.1016/0021-8693(86)90050-5. MR836629 (87i:46142)

[Han87] David E. Handelman, Positive polynomials, convex integral polytopes, and a random walk problem, Lecture Notes in Mathematics, vol. 1282, Springer-Verlag, Berlin, 1987. MR 914972 (89b:06014)

[Han88] David Handelman, Representing polynomials by positive linear functions on compact convex polyhedra, Pacific J. Math. 132 (1988), no. 1, 35-62. MR.929582 (90e:52005)

[Han95] David Handelman, Iterated multiplication of characters of compact connected Lie groups, J. Algebra 173 (1995), no. 1, 67-96, DOI 10.1006/jabr.1995.1078. MR.1327361 (96c:22005)

[Han13] David Handelman, Simple Archimedean dimension groups, Proc. Amer. Math. Soc. 141 (2013), no. 11, 3787-3792, DOI 10.1090/S0002-9939-2013-11672-2. MR3091768

[H7] David Handelman, Real dimension groups. Canadian Math Bull. 56 (2013), no. 3, 551-563. MR3078224

[HPS92] Richard H. Herman, Ian F. Putnam, and Christian F. Skau, Ordered Bratteli diagrams, dimension groups and topological dynamics, Internat. J. Math. 3 (1992), no. 6, 827-864, DOI 10.1142/S0129167X92000382. MR1194074 (94f:46096)

[Kar12] O. Karpel, Infinite measures on Cantor spaces, J. Difference Equ. Appl. 18 (2012), no. 4, 703-720, DOI 10.1080/10236198.2011.620955. MR.2905292

[KRW] K. H. Kim, F. W. Rousch, S. G. Williams, Duality and its consequences for ordered cohomology of finite type shifts, Combinatorial and computational mathematics, Pohang (Korea), 2001.

[L] John Lawrence, A simple torsion-free perforated interpolation group, unpublished, ca 1981 .

[Law84] John Lawrence, Countable abelian groups with a discrete norm are free, Proc. Amer. Math. Soc. 90 (1984), no. 3, 352-354, DOI 10.2307/2044471. MR728346 (85i:20056)

[Mat02] Hiroki Matui, Dimension groups of topological joinings and non-coalescence of Cantor minimal systems, Pacific J. Math. 204 (2002), no. 1, 163-176, DOI 10.2140/pjm.2002.204.163. MR.1905196(2003b:37018)

[Med06] Konstantin Medynets, Cantor aperiodic systems and Bratteli diagrams (English, with English and French summaries), C. R. Math. Acad. Sci. Paris 342 (2006), no. 1, 43-46, DOI 10.1016/j.crma.2005.10.024. MR2193394(2006g:37011)

[Mum76] David Mumford, Algebraic geometry. I, Springer-Verlag, Berlin-New York, 1976. Complex projective varieties; Grundlehren der Mathematischen Wissenschaften, No. 221. MR0453732 (56 \#11992)

[Poo89] Yiu Tung Poon, A K-theoretic invariant for dynamical systems, Trans. Amer. Math. Soc. 311 (1989), no. 2, 515-533, DOI 10.2307/2001140. MR978367(90c:46091)

[Put89] Ian F. Putnam, The $C^{*}$-algebras associated with minimal homeomorphisms of the Cantor set, Pacific J. Math. 136 (1989), no. 2, 329-353. MR978619 (90a:46184)

[Ver81] A. M. Vershik, Uniform algebraic approximation of shift and multiplication operators (Russian), Dokl. Akad. Nauk SSSR 259 (1981), no. 3, 526-529. MR625756 (83c:46064) 
[Yin08] Andrew Q. Yingst, A characterization of homeomorphic Bernoulli trial measures, Trans. Amer. Math. Soc. 360 (2008), no. 2, 1103-1131 (electronic), DOI 10.1090/S0002-9947-07-04431-5. MR2346485 (2009a:28034)

Institute for Low Temperature Physics, 47 Lenin Avenue, 61103 Kharkov, Ukraine

E-mail address: bezuglyi@ilt.kharkov.ua

Department of Mathematics, University of Ottawa, Ottawa, Ontario K1N 6N5, Canada

E-mail address: dehsg@uottawa.ca 